Joëlle Bahloul

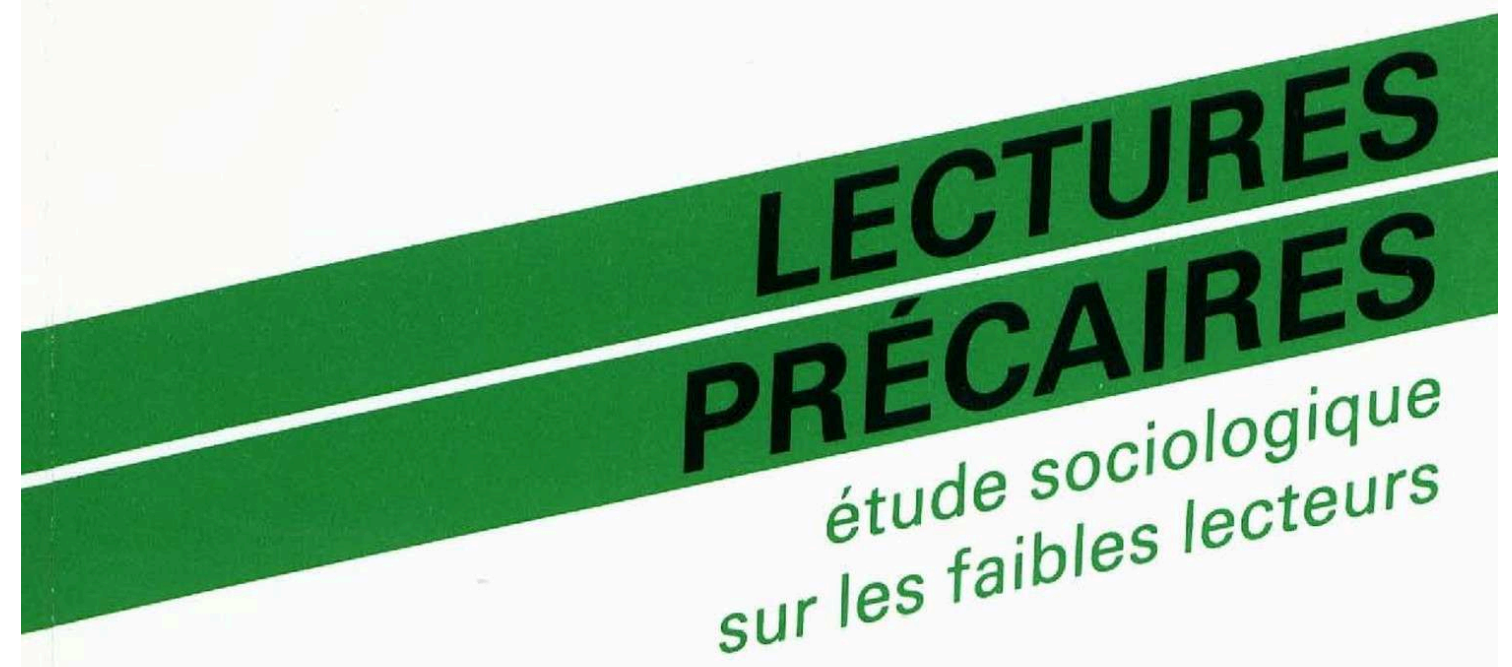

Service des études et de la recherche Bibliothèque publique d'information 瑟Centre Georges Pompidou 


\section{Lectures précaires}

Études sociologiques sur les faibles lecteurs

Joëlle Bahloul

DOI : 10.4000/books.bibpompidou. 1755

Éditeur : Éditions de la Bibliothèque publique d'information Année d'édition : 1990

Date de mise en ligne : 5 juin 2015

Collection : Études et recherche

ISBN électronique : 9782842461768

\section{Doneftition ebooks}

http://books.openedition.org

\section{Édition imprimée}

ISBN : 9782902706167

Nombre de pages : 128

\section{Référence électronique}

BAHLOUL, Joëlle. Lectures précaires : Études sociologiques sur les faibles lecteurs. Nouvelle édition [en ligne]. Paris : Éditions de la Bibliothèque publique d'information, 1990 (généré le 02 février 2021).

Disponible sur Internet : <http://books.openedition.org/bibpompidou/1755>. ISBN : 9782842461768. DOI : https://doi.org/10.4000/books.bibpompidou. 1755.

Ce document a été généré automatiquement le 2 février 2021. Il est issu d'une numérisation par reconnaissance optique de caractères.

(c) Éditions de la Bibliothèque publique d'information, 1990

Conditions d'utilisation:

http://www.openedition.org/6540 
La coupure entre les lecteurs et les non lecteurs n'est pas aussi radicale qu'on le croit. On n'a jamais étudié jusqu'ici la faible lecture, ce rapport fragile au livre, suspendu entre le doute et le désir.

Cette recherche sociologique originale analyse les pratiques effectives de.lecture et les représentations sociales du livre souvent contradictoires : on valorise le livre, on dénie ses lectures : «je ne lis pas, je ne lis que de petits romans ». "Quand je Iis de grands auteurs j'ai l'impression que ça m'apporte plus ».

Qu'en est-il des temps et modes de la lecture chez ces lecteurs fragiles? Qu'en est-il du rôle des sociabilités autour de la lecture? Qu'en est-il des modalités de choix, de l'établissement des préférences?

Cette étude fait apparaître les caractéristiques paradoxales d'une pratique qui ne "capitalise " pas sur le plan culturel, s'effectue souvent selon des modes ponctuels, non structurés, irréguliers mais qui témoigne chez ceux qui s'y livrent d'une croyance symbolique forte dans le rôle du livre.

\section{JOËLLE BAHLOUL}

Joëlle Bahloul est ethnologue, professeur assistant du département d'anthropologie de l'Indiana University (États-Unis). 
SOMMAIRE

\author{
Avertissement \\ Introduction. Lecture et lectures \\ De l'utilité méthodologique des questions « Qui est faible lecteur? » et «Comment le trouver? » \\ De la signification méthodologique de la question « Combien de livres lisez-vous dans l'année?» \\ Ce que les lecteurs entendent par « lire» \\ Ce que les lecteurs entendent par « livre» \\ L'approche qualitative de la sociologie de la lecture \\ Biographie familiale et éducative et biographie lectorale \\ La socialisation de la lecture \\ Représentations du livre et de la lecture
}

\title{
Préambule. Scénarios « en chiasme »
}

Trajectoires sociales et scénarios de lecture : quelques cheminements

La faible lecture et la trajectoire scolaire

Mobilité socioprofessionnelle et scénario de lecture

« Accidents » de parcours lectoraux

\section{Première partie. Le livre en pratiques}

L'information sur les livres et l'acquisition des livres

Livres possédés, livres stockés et rangés, livres liquidés

\section{Deuxième partie. La lecture en pratiques}

La lecture, une question de temps

La lecture, une question de genres

Le déchiffrement du texte dans la faible lecture

\section{Troisième partie. Le livre et la lecture : perception, modélisation, représentation}

Le lecteur, un « professionnel » de la lecture

La lecture : apprendre, se détendre, s'évader, définition fonctionnelle

Le livre : définitions matérielle, graphique, par le genre ou par l'auteur

\section{Conclusion}

\section{Annexes}

\section{Les enquêtes sur la faible lecture}

La méthode

Entretien $n^{\circ} 5$ : Faible lectrice, 19.5.85

L'échantillon (voir ci-après, la liste des personnes interviewées)

\section{Liste des personnes interviewées}

\section{Plan d'entretien}

Le livre et la lecture : symbolique et représentations

Pratique personnelle de la lecture

Plan d'observation ethnographique

Renseignements personnels

Conditions d'entretien 


\section{Extraits d'entretiens}

Entretien 1

Entretien 17

Entretien 26

Entretien 27

Entretien 30

Entretien 33

Entretien 36

Entretien 38

Entretien 41

Entretien 46

Bibliographie 


\section{Avertissement}

1 Cette étude a été financée à la demande de la Direction du livre et de la lecture par le Département des études et de la prospective du ministère de la Culture et de la Communication.

2 Le suivi scientifique de l'étude a été assuré conjointement par le Département des études et de la prospective et par le Service des études et de la recherche de la Bibliothèque publique d'information du Centre Pompidou.

3 Cette recherche a été réalisée dans le cadre de l'association ADRESSE (Association pour le développement des recherches et études sociologiques et économiques).

4 Je remercie,

5 Janet Morford, Catherine Saulnier, Bernard Urlacher et Denis Vi-dal-Naquet qui m'ont aidée dans la collecte des interviews,

6 Jean-François Barbier-Bouvet, Martine Poulain, Marc Petit et François Rouet qui ont suivi le déroulement de cette étude et m'ont prodigué de précieux conseils,

7 Nathalie Heinich dont la lecture attentive du manuscrit a permis des rectifications nécessaires,

8 Denise Cheminant et Françoise Daudin qui ont bien voulu assurer l'élaboration matérielle de l'étude. 


\section{Introduction. Lecture et lectures}

1 De la construction de son objet à la collecte de l'analyse des matériaux, la sociologie de la lecture ne peut se priver de l'exploration, par le sociologue lui-même, de son propre rapport à la lecture, de sa pratique et des modèles qui façonnent sa représentation du livre et de la lecture. Le sociologue est un lecteur, doté de ses modèles pratiques et idéologiques; il se présente d'emblée à son objet armé de ses présupposés culturocentristes. Sur le terrain, il ne peut éviter d'apparaître aux yeux de ses interlocuteurs comme le détenteur de la légitimité d'interroger une pratique dont il maîtrise le modèle dominant. La distanciation scientifique qu'exigerait toute approche sociologique des pratiques de lectures paraît particulièrement âpre sur un terrain où le sociologue est lui-même si activement impliqué. Elle constitue un préalable épistémologique qui rend nécessaires la critique des documents d'enquête collectés, et le réajustement constant des hypothèses et de la démarche.

2 Cette exigence apparaît d'autant plus impérieuse lorsque l'enquête de sociologie de la lecture est gérée par une demande du ministère de la Culture qui la formule, comme dans le cas de la présente étude, dans le cadre d'une politique générale de lutte contre l'affaiblissement des pratiques de lectures :

3 «Des freins considérables subsistent sur lesquels il faut agir (...). Réapparition d'une perte de la capacité de lire («illettrisme »), blocages dus aux conditions de vie et de travail, à l'appartenance culturelle, disparition trop souvent du plaisir de lire au profit d'un simple apprentissage de la lecture ${ }^{1}$ "

4 La présente étude de sociologie d'une pratique lectorale s'inscrit donc manifestement dans un processus historique et idéologique particulier de lutte contre l'illettrisme croissant mis en exergue dans les rapports publiés ces dernières années par les services conjoints du ministère de la Culture et du ministère de la Solidarité nationale ${ }^{2}$. C'est dans ce contexte qu'il faut interpréter la formulation de la demande de la Direction du livre qui suggère l'exploration des pratiques de "faible lecture" en termes d'«obstacles à la lecture». La demande portait sur l'exploration qualitative et psychosociologique "des démarches de lecture fragilisées par la situation sociale des individus concernés (...), des démarches cumulatives et différenciantes (...) appelant fortement les représentations tant du livre que de l'individu lisant ${ }^{3}$ ». Cette perspective négativiste proposée dans le projet d'étude suggérait en fait un modèle de lecture légitimé par le rédacteur lui-même, à savoir l'institution culturelle représentée par le 
ministère et par la Direction du livre. Ce modèle était précisé dans la justification de la demande: on mettait en exergue une population spécifiée de lecteurs "faibles", produit d'une typologie construite sur la base de variables statistiques, à savoir le nombre déclaré de livres lus dans l'année, soit un à quatre livres et au maximum neuf livres.

$5 \quad$ L'approche statistique de la sociologie de la lecture est de loin la plus répandue parmi les quelques deux cent dix enquêtes réalisées depuis 1955. On observe cependant dans cette riche panoplie d'outils statistiques des variations sensibles dans la construction des questionnaires et des échelles de quantification de la lecture; au sujet de ces dernières, on enregistre également des variations dans la détermination des périodes de quantification : on questionnera sur les lecteurs ou les achats de livres effectués soit dans la dernière semaine ou dans le dernier mois, soit dans le dernier trimestre ou dans les derniers six mois, soit encore dans l'année écoulée. De ce fait, les résultats de ces sondages se prêtent difficilement à la comparaison et font d'ailleurs apparaître des divergences sensibles dans la quantification. Dans ces conditions l'approche statistique de la lecture semble n'autoriser que la mise en évidence de tendances générales mais ne permet ni une estimation ni une construction exhaustives de la typologie du lectorat français.

6 La méthodologie employée dans la réalisation de la dernière enquête sur les Pratiques culturelles des Français ${ }^{4}$ et sur laquelle est fondée la demande de la présente étude, vient uniformiser les critères et la périodisation de la quantification: les statistiques recueillies portent sur les livres lus pendant l'année écoulée et sont découpées selon l'échelle suivante : d'un modèle légitimé par le critère quantitatif : le meilleur lecteur est censé être celui
qui lit le plus grand nombre de livres, quel que soit le type de publications, la forme de
la lecture et son usage social et culturel. D'autre part, cette modélisation statistique des seuils d'appréciation de la lecture : on est « faible » lecteur lorsqu'on lit un à quatre livres (et jusqu'à neuf livres) par an, " moyen » lecteur lorsqu'on lit dix à vingt-quatre livres par an, "gros » lecteur lorsqu'on lit plus de vingt-cinq livres par an. Cette modélisation quantitative ne s'accompagne d'aucune justification du positionnement des seuils : 1, 4, 9, 24, plus de 25. On ne peut s'empêcher d'y voir une légitimation, sous forme quantitative, d'une certaine pratique lectorale, ici instituée en modèle qui consisterait à privilégier une accumulation quantitative de lecture, quels qu'en soient le rythme et la forme.

12 C'est dans cette logique qu'il faut réinscrire la formulation négative de la demande d'étude de la faible lecture : considérée comme un « obstacle » à la lecture, et de ce fait associée à l'abandon ou à la fragilité de certaines pratiques lectorales, la faible lecture en augmentation dans la dernière décennie est interprétée d'emblée comme un mécanisme de réduction ou de fragilisation de pratiques de lecture plus soutenues. l'approche et dans les techniques envisagées, ouvre une perspective qui a pu nous lapproche et dans les techniques envisagées, ouvre une perspective qui a pu nous 
apparaître, au moment de l'investigation sur le terrain, assez éloignée des typologies statistiques sur lesquelles elle se fondait.

\section{De l'utilité méthodologique des questions «Qui est faible lecteur ? » et « Comment le trouver ? »}

La demande de délimitation de notre champ d'investigation paraissait simple: il s'agissait de constituer un réseau d'informateurs stratégiquement représentatifs $d u$ faible lectorat, c'est-à-dire composé de lecteurs de un à quatre livres par an, et au maximum de neuf livres par an.

Le repérage sociologique du faible lectorat, effectué par le Service des études et recherches du ministère de la Culture dans le cadre de l'enquête sur les Pratiques culturelles des Français $^{6}$ fournit des indications qui mettent en relief certaines spécificités démographiques et socioprofessionnelles. Les $27,9 \%$ de lecteurs de un à neuf livres par an se distinguent par une légère prédominance masculine, tandis que les femmes sont majoritaires parmi les non-lecteurs. Ils disposent d'un niveau d'instruction moyen ou faible, dépassant rarement le brevet élémentaire du premier cycle (BEPC) ; dans leur grande majorité, ils ont interrompu leur scolarité aux alentours de l'âge de 15 ans ou auparavant.

Les caractéristiques relatives à l'âge des lecteurs ne sont pas aussi saillantes: on observe que les lecteurs de un à quatre livres sont en majorité âgés de 25 à 29 ans, une tranche d'âge assez étendue recouvrant des scénarios éducatifs très divers. En revanche, les lecteurs de cinq à neuf livres sont beaucoup plus jeunes puisqu'on y trouve une forte pénétration des 15-24 ans (30\%).

17 La répartition socioprofessionnelle des faibles lecteurs est plus parlante. Elle recoupe les données concernant leur niveau d'instruction, pour le groupe des lecteurs de un à quatre livres surtout: à la faiblesse des qualifications correspond une forte implantation de la catégorie dans les groupes socioprofessionnels placés au bas de l'échelle sociale, ouvriers qualifiés et spécialisés, manœuvres et personnels de service, contremaîtres et aussi une bonne proportion de petits agriculteurs. Les lecteurs de cinq à neuf livres se recrutent dans les fractions sociales plus élevées, employés pour la plupart, et comportent aussi un grand nombre de femmes au foyer. Distincts au plan de la catégorie socioprofessionnelle et de l'origine sociale, les faibles lecteurs le sont également à celui de la répartition géographique puisqu'ils résident essentiellement dans des communes rurales ou dans des villes de moins de 100000 habitants, c'est-àdire dans les régions où la distribution du livre reste encore limitée.

Les faibles lecteurs semblent donc constituer une catégorie qui se distingue par sa position dans la hiérarchie socioprofessionnelle et par son niveau d'instruction. Pour autant, ces spécificités nous fournissent des informations sommaires sur les "scénarios » de lecture, les représentations du livre et de la lecture, la modélisation des genres et les pratiques d'acquisition du livre, les mécanismes psychotechniques de la lecture, la socialisation de la lecture, la place du livre dans l'univers matériel, et celle de la lecture dans les biographies éducatives et familiales.

19 Il nous a paru intéressant, plutôt que de conformer notre échantillon d'enquêtes aux spécificités sociodémographiques et socioéducatives du "faible lectorat », d'élargir notre recrutement à l'ensemble de l'espace socioprofessionnel, de diversifier les 
trajectoires socio éducatives et les niveaux d'instruction, afin d'avoir accès à la variété des scénarios de lecture et surtout d'en faire apparaître la fonction diachronique: comme bien des pratiques culturelles, la lecture ne constitue par un donné de la condition sociale; elle répond aux circonvolutions complexes de la biographie socioéducative, socio professionnelle et familiale. Nous entendons donc par "scénario de lecture " l'ensemble des conditions sociales produites par l'histoire familiale, socioprofessionnelle et éducative des lecteurs.

20 Nos contacts de pré-enquête avaient mis l'accent sur la diversité des trajectoires des faibles lecteurs : pour être faible lecteur, on peut avoir suivi des formes évolutives de pratiques, avoir plus ou moins lu, modifié ses préférences en genre et en types d'imprimés, son mode de lecture. C'est pour accéder à ces mécanismes évolutifs que nous avons choisi de diversifier notre recrutement des personnes à interroger ${ }^{7}$. De ce point de vue, notre réseau d'informateurs constitue moins un échantillon statistiquement représentatif qu'un regroupement de scénarios constituant des variables stratégiques.

21 L'approche diachronique de la faible lecture pouvait en outre fournir des éléments de réponse à une autre question fondamentale : d'où provient l'accroissement du nombre des faibles lecteurs entre 1973 et 1981 ? Les rangs du faible lectorat ont-ils été approvisionnés par la réduction des moyens ou gros lecteurs ou par celle des nonlecteurs? Bref, comment devient-on faible lecteur? Est-on faible lecteur après avoir diminué ses lectures ou bien en ayant progressivement accès à la lecture après n'avoir lu aucun livre pendant une longue période? Comment s'aménage, dans ces mécanismes, le rapport des pratiques à l'origine sociale, aux acquis scolaires et pour ce dernier point, observe-t-on dans la génération du baby-boom un effet de l'accès massif au système scolaire? La question est importante: elle permet de justifier ou de contredire l'approche négativiste généralement admise de la faible lecture. L'analyse des données partielles de l'enquête sur Les Pratiques culturelles des Français permet d'avancer certaines hypothèses concernant cette question fondamentale 8 .

- L'accroissement du nombre des faibles lecteurs entre 1973 et 1981 s'accompagne d'une diminution proportionnelle de celui des non-lecteurs.

- On peut estimer qu'il existe une faible probabilité d'abandon chez les gros lecteurs, et ce pour les raisons suivantes :

24 - La progression du faible lectorat est surtout le résultat de l'apport de catérogies sociales en général bien représentées parmi les non-lecteurs et les faibles lecteurs; à ce sujet on s'appuiera sur la forte augmentation des faibles lecteurs chez les agriculteurs, les ouvriers qualifiés et spécialisés, les manœuvres et les contremaitres (plus de $11 \%$ pour les agriculteurs, plus de 9,9\% pour les autres). Dans le même ordre d'idées, cet accroissement $\mathrm{du}$ faible lectorat est aussi en grande partie assuré par l'apport substantiel des titulaires de qualifications moyennes voire rudimentaires (certificat d'aptitude professionnelle [CAP], certificat d'études primaire [CEP], BEPC), population elle aussi spécifique du petit et du non-lectorat. Les catégories de gros lecteurs ont très peu participé à l'augmentation du faible lectorat, sauf les cadres supérieurs et les professions libérales (plus de 8,6\% en pénétration), et les résidents des villes de plus de 100000 habitants (plus de $8,2 \%$ en pénétration). En ce qui concerne cette dernière donnée, on observera cependant que les faibles lecteurs résident essentiellement dans les villes de moins de 100000 habitants (58,7\%), agglomérations dont les composantes et la structure sociale contribuent à la faible diffusion du livre. 
- Les genres littéraires les plus fréquemment lus par les faibles lecteurs ${ }^{9}$, ouvrages pratiques, encyclopédies, romans (autres que les policiers et les romans d'espionnage) sont pour certains en promotion sensible depuis quelques années, faciles d'accès et d'une manière générale moins fréquentés par les lecteurs dont la pratique est plus diversifiée et intensive.

L'ensemble de ces données permet de renverser la perception négativiste du faible lectorat: l'accroissement de cette pratique entre les deux enquêtes des Pratiques culturelles semble en effet provenir essentiellement de la réduction des non-lecteurs; l'amplification d'une lecture "faible » en quantité constitue donc un mécanisme de développement et non de fragilisation de la lecture. Dans ces conditions, il apparaissait nécessaire de tenir compte, dans le recrutement de nos informateurs, non seulement de l'appartenance socio-professionnelle ou socio-éducative mais aussi des modalités biographiques de la pratique lectorale: origine sociale, capital culturel familial, formation intellectuelle et professionnelle, insertion sociale, etc. Il devenait nécessaire de diversifier également les scénarios de lecture.

\section{De la signification méthodologique de la question «Combien de livres lisez-vous dans l'année?»}

27 Après avoir procédé à une sélection diversifiée de nos recrutements, tant par les critères sociodémographiques et socioprofessionnels que par les scénarios, nous retombions dans les avatars de la typologie statistique et quantitative des pratiques lectorales lorsque pour sélectionner nos interviewés - lecteurs de 1 à 4 livres par an nous devions poser la question préalable: «Combien de livres lisez-vous dans l'année?» La question nous renvoie à ce que Pierre Bourdieu appelle "l'effet de légitimité » :

«Dès qu'on demande à quelqu'un ce qu'il lit, il entend: qu'est-ce que je lis qui mérite d'être déclaré? C'est-à-dire: qu'est-ce que je lis en fait de littérature légitime ${ }^{10}$ ?»

C'est dans la formulation quantitative de l'interrogation et dans l'emploi de la terminologie du livre qu'il faut trouver l'effet de légitimité de notre question sélective. On pourrait donc, à l'appui de nos observations sur le terrain concernant les réactions à cette question, ajouter les détails suivants aux interprétations de Pierre Bourdieu sur l'effet de la légitimité de la question : le « combien de livres lisez-vous » était interprété comme suit :

«Quelle quantité de livres dois-je déclarer qui puisse être considérée comme la pratique légitime du lecteur» ou bien «quelles sortes d'imprimés est-ce que je lis qui puissent être compris dans la catégorie « livre »?"

Ainsi le travail de l'enquêteur-sélectionneur s'ouvrait sur ce préambule dans lequel les personnes interrogées indiquaient déjà leur propre modélisation et de la lecture et du livre. Et l'enquêteur était contraint dans bien des cas d'explorer ces modélisations pour conformer sa sélection à la typologie quantitative initiale. 


\section{Ce que les lecteurs entendent par « lire »}

fil de son exploration introductive que son interlocuteur lit un à deux ouvrages pendant les vacances et au hasard de ses intérêts ponctuels. « Mais je n'appelle pas ça lire » ajoute l'interviewé. Cette fréquence et ce rythme de lecture équivalent pour lui à une non-lecture. D'autres modélisations ont trait au type de fréquentation des livres : les lectures en consultation des encyclopédies ou des ouvrages pratiques sont fortement dévalorisées : «Je n'appelle pas ça lire!» nous dit-on. Enfin une approche fonctionnaliste de la lecture aura tendance à mésestimer les consultations ponctuelles et partielles d'ouvrages à usage purement professionnel: c'est le cas de certains lecteurs, techniciens, enseignants, avocats ou autres professions libérales qui considèrent que cet usage très ciblé de l'imprimé ne constitue pas une lecture, celle-ci étant classée au registre des activités de loisirs et de détente.

31

Finalement, ceux qui lisent de manière fortuite, en consultation partielle et ponctuelle ou bien professionnelle, se présenteront d'emblée comme des non-lecteurs, ayant convenu que ces formes de lecture ne sont pas légitimées par le questionnement de l'enquêteur, formulé en termes quantitatifs, la présentation générique du "combien " et du « lire des livres » appelle des réponses sélectives et non différentielles.

\section{Ce que les lecteurs entendent par « livre »}

L'un des avatars du questionnement sélectif de nos faibles lecteurs est qu'il est censé établir une estimation de la pratique lectorale par le recensement de la lecture exclusive des livres. Il exclut de ce fait les lecteurs de presse - quotidiens, magazines et revues - parmi lesquels on trouve beaucoup de non-lecteurs de livres, et qui sont néanmoins d'assidus lecteurs de presse. On négligerait une dimension essentielle de la lecture en distinguant lecture de presse et lecture de livres ${ }^{11}$, instituant cette seconde pratique en modèle de lecture, et concluant au décompte des seuls lecteurs de livres dans la quantification du lectorat. La lecture exclusive de presse peut constituer dans certains cas une alternative ou une compensation à la lecture de livres, par la forme de connaissance discontinue qu'elle fournit, mais n'en exige pas pour autant moins de compétences psychotechniques et de déchiffrage. La question sur la quantification de la pratique lectorale, étant centrée sur la notion de "livre", a tendance à produire des effets de minorisation et de dévalorisation de leurs pratiques lectorales par les lecteurs exclusifs de presse, qui se présentent le plus souvent comme des non-lecteurs.

Outre cette distinction entre livre et presse, le questionnement sur la lecture suggère l'hypervalorisation de la catégorie « livre ». La plupart des interviewés y entendaient les romans essentiellement, et accessoirement les récits, essais historiques et livres d'actualité. La terminologie du livre sélectionne les genres littéraires: les bandes dessinées sont ainsi fortement dévalorisées pour la prédominance accordée à l'image.

D'autres sélections opèrent dans la forme éditoriale de l'imprimé : on entend par livre un ouvrage broché ou "cartonné ». Cette modélisation tend à exclure de la catégorie les ouvrages de diffusion professionnelle et technique limitée, présentés en forme de documents multigraphiés. En revanche, certaines collections de revues - scientifiques 
ou culturelles - conservées et parfois reliées, peuvent être intégrées à la catégorie du livre $^{12}$.

35 Les terminologies de la lecture comportent des pièges et des avantages méthodologiques : elles mettent à nu des représentations du livre et de la lecture, mais en même temps exigent la critique permanente des informations lorsque celles-ci ont pour objectif de construire des typologies. Parce que les personnes interviewées situent l'enquêteur dans une position hiérarchique à laquelle ils associent les modèles dominants de la pratique lectorale, leurs réponses s'inscrivent d'emblée dans ces modèles pour aboutir à la dévalorisation de leurs pratiques. Cela est d'autant plus vrai que nous avons en l'occurrence enquêté dans un milieu de faibles lecteurs où la conscience de cette hiérarchisation est actualisée par l'arrivée de l'enquêteur : on sait qu'il est étudiant ou qu'il est "chargé d'études" et on le classe d'emblée dans la catégorie des intellectuels gros consommateurs de littérature légitime. Ainsi dans le déroulement des interviews, la question «Combien de livres lisez-vous en moyenne dans l'année?» a pu être interprétée progressivement ou expressément par l'interrogation suivante: «Pourquoi ne lisez-vous pas plus?» ou bien « N'avez-vous jamais lu un seul roman?».

\section{L'approche qualitative de la sociologie de la lecture}

Ces mésaventures d'une enquête fondée sur des catégories statistiques nous ont contraints à reconsidérer ces typologies avec la nécessité critique que suggéraient les observations sur le terrain. Nous avions affaire tantôt à des non-lecteurs de livres dévorant les colonnes des quotidiens - tantôt à des petits lecteurs de vacances se présentant comme des non-lecteurs absolus, tantôt à des lecteurs exclusifs de documents techniques et professionnels s'excluant de notre champ d'observation du "faible lectorat", estimant que "ça n'est pas de la lecture ", tantôt à des lectrices exclusives de la collection Harlequin renchérissant sur leur goût assoiffé pour la lecture. Le faible lectorat et la lecture en général recouvrent des pratiques et des représentations différenciées. Il y a lecture et lectures, et comme le souligne Pierre Bourdieu, à la formulation générique ou statistique de la lecture, «il faut substituer l'idée de lectures au pluriel et l'intention de chercher des indicateurs des manières de lire ${ }^{13} »$.

37 Les difficultés techniques de l'enquêteur à la recherche d'un «vrai » faible lecteur se sont transformées en données informatives sur ce qui sera probablement l'hypothèse initiale de notre étude : la faible lecture, même si elle est généralement appréhendée conformément aux typologies statistiques ${ }^{14}$, est ici envisagée en termes qualitatifs ; on recherchera en effet les mécanismes pratiques et idéologiques qui constituent cette approche de la lecture et qui peuvent s'observer dans des catégories statistiques différentes, celle en particulier de grands lecteurs exclusifs.

Cette perspective qualitative et sociologique de l'étude du faible lectorat appréhende la lecture comme une pratique sociale à part entière, et non plus seulement comme une pratique purement culturelle. Parce qu'elle s'inscrit dans la complexité des interactions et des échanges qui composent la socialisation du lecteur, elle sera explorée dans cette totalité, et dans la perspective maussienne, comme « fait social total ». Cette démarche suggère la mise en perspective historique d'une pratique dont la logique est à chercher dans la biographie familiale et éducative du lecteur mais également dans l'ensemble des 
pratiques de socialisation de la lecture et des représentations du livre. Notre plan de questionnement a donc été construit selon les trois axes suivants ${ }^{15}$.

\section{Biographie familiale et éducative et biographie lectorale}

39 Parce que la faible lecture ne constitue pas un «donné » sociologique et pas plus ou moins que la «non-lecture» ou la "grande lecture», elle ne peut être envisagée comme un a priori de l'identité sociale : autrement dit, la faible lecture ne représente pas une catégorie sociale homogène. Elle s'inscrit nécessairement dans la diachronie des conditions sociales du lecteur. L'approche biographique offre donc la possibilité de reconstituer les divers scénarios qui ont abouti à la faible lecture; en même temps elle replace la pratique lectorale « dans son horizon culturel de référence » et « dessine (les circonvolutions) de la mouvance culturelle » du lecteur ${ }^{16}$.

L'approche biographique et diachronique de cette pratique spécifique de la lecture permet d'explorer, à l'instar des analyses de Pierre Bourdieu des pratiques culturelles, «la relation très étroite qui unit (ces dernières) au capital scolaire (mesuré aux diplômes obtenus) et, secondairement, à l'origine sociale (saisie au travers de la profession du père $)^{17} »$.

\section{La socialisation de la lecture}

41 La lecture n'est pas pratique sociale uniquement parce qu'elle classe ou est classée dans la hiérarchie des statuts sociaux. Elle donne lieu à des interactions et échanges sociaux. Elle n'est pas non plus, comme l'affirme la représentation traditionnelle, acte de pure intimité ou de retranchement individualiste à l'écart du monde et de la société. La lecture est totalement impliquée dans l'organisation et les conditions sociales. L'initiative de la lecture, la réception et la circulation des connaissances acquises, les représentations du livre et $\mathrm{du}$ lire opèrent dans le cadre des "réseaux» de socialisation. En bref, il s'agit ici d'explorer le tissu social dans lequel se déploient les faibles pratiques de lecture. Comment prend-on l'initiative de lire? Quelle est la source stimulante? Comment acquiert-on les livres que l'on lit? Que deviennent les livres acquis? Les lit-on ou les conserve-t-on dans sa bibliothèque à titre purement démonstratif? Quand et où lit-on? Quel type de lecture pratique-t-on? C'est à une sorte d'archéologie de la pratique lectorale que nous nous sommes prêtés. L'approche nous permet par exemple de déchiffrer la part de " capital culturel objectivé18 » qui est investie dans la lecture et qui souvent rend compte de sa totalité sociologique. Les formes d'appropriation ou d'expropriation du "capital livresque » s'articulent déjà dans le rapport matériel au livre : la possession ou non d'un stock de livres, la forme et les lieux du rangement, le volume occupé par le livre-objet dans l'univers domestique, la gestualité de la lecture sont autant d'indicateurs non seulement du statut matériel du livre mais aussi de son statut idéologique et social.

Enfin l'un des aspects et non des moindres de l'approche qualitative de la lecture a consisté à se poser la question des genres et de leur évaluation. Peut-on en effet classer dans la même catégorie de "grands lecteurs » les lectrices assoiffées et exclusives de la collection Harlequin, aux côtés des lecteurs d'une gamme diversifiée de genres 
littéraires, allant du roman de littérature classique à l'essai politique, en passant par la bande dessinée, le roman policier ou la science-fiction? On a vu que les démarches s'imprimaient dans l'évaluation et la modélisation des genres. Il y a des genres légitimes et d'autres qui sont marginalisés. Il conviendra de s'interroger sur l'écart qui sépare ces évaluations, chez les faibles lecteurs, de leurs pratiques réelles, de leurs préférences et de leur sélection des genres lus.

Comme l'a montré Patrick Parmentier dans une étude sur la lecture ${ }^{19}$, chaque genre littéraire est doté de son statut social. Les classements du livre et de l'imprimé sont régis par la distribution du capital social et culturel. Les textes classés sont aussi des classificateurs sociaux. On observait déjà ces mécanismes dans les résultats des Pratiques culturelles sur les genres littéraires préférés des faibles lecteurs ${ }^{20}$. Ils sont les lecteurs les plus assidus d'ouvrages pratiques, des dictionnaires, des encyclopédies et des romans autres que les policiers et les romans d'espionnage.

\section{Représentations du livre et de la lecture}

Des échantillons substantiels de ces représentations nous étaient déjà fournis dans les toutes premières phases de nos enquêtes, lors de la sélection statistique de nos informateurs. Ils livraient d'emblée les repérages de la pratique légitime, la pratique instituée en modèle dominant. Pour autant, il existe des représentations pratiques, chez les faibles lecteurs, qui pourraient indiquer des formes de légitimation d'un modèle différentiel de lecture, caractérisé par des formes spécifiques d'accès à la culture de masse, médiatique et médiatisée. En d'autres termes, les faibles lecteurs développent des pratiques de lecture chargées de représentations parfois contradictoires de celles imprimées dans la logique du discours dominant qu'ils ont assimilé.

Le faible lectorat dispose-t-il de stratégies pratiques d'appropriation de ses pratiques lectorales comme capital culturel légitime ? Les livres lus sont-ils par lui reconnus et de quelle manière, comme vecteurs de légitimation d'habitus culturels qui constitueraient le modèle différentiel apparemment formulé ?

\section{NOTES}

1. Gattegno Jean, Bilan d'une action : la politique de la Direction du livre. Pratiques de la lecture, sous la direction de Roger CHARTIER. - Paris : Rivages, 1985. p. 215.

2. BÉNICHOU Jean-Pierre, ESPÉRASDIEU Véronique. LION Antoine. Des illettrés eu France. - Paris : Documentation française. 1984.

3. Proposition de recherche sur les obstacles à la lecture, ministère de la Culture, Service des études et recherches.

4. Pratiques culturelles des Français, évolution 1973-1981, ministère de la Culture. Service des études et recherches. - Paris : Dalloz, 1982.

5. Pratiques culturelles..., op. cit.. p. 72. 
6. Brefs repères sur les faibles lecteurs, A Données complémentaires sur les non-lecteurs, les faibles lecteurs et les gros lecteurs, ministère de la Culture. Service des études et recherches. mai 1984.

7. Voir description du réseau d'informateurs en annexes.

8. Brefs repères sur les faibles lecteurs, op. cit.

9. Données complémentaires..., op. cit.

10. BOURDIEU Pierre et CHARTIER Roger, La lecture : une pratique culturelle, Pratiques de la lecture, op. cit., p. 223.

11. Cette distinction est assez rigoureuse dans l'enquête sur les Pratiques culturelles des Français.

12. Pour cette raison également, la distinction entre le livre et la presse apparaît inopérante dans la quantification de la lecture.

13. BOURDIEU Pierre, op. cit.. p. 223.

14. La plupart des personnes interviewées lisent un à quatre ou neuf livres au maximum et en moyenne dans l'année. Pour les besoins de la comparaison nous avons également interrogé quelques grands lecteurs et non-lecteurs. Voir les détails de l'enquête dans les annexes.

15. Voir détails du plan d'entretien en annexes p. 108

16. HÉBRARD Jean, Comment Valentin Jamerey Duval apprit-il à lire : l'autodidaxie exemplaire. Pratiques de la lecture, op. cit. p. 29.

17. BOURDIEU Pierre, La Distinction. - Paris : Minuit. 1979. p. 12.

18. BOURDIEU Pierre. Les trois états du capital culturel. Actes de la recherche en sciences sociales, $\mathrm{n}^{\circ}$ 30,1979, p. 5.

19. PARMENTIER Patrick, Les genres, le classement des lecteurs et le classement des livres, Trois études sur la lecture, enquêtes et analyses réalisées par le Gides, 1981.

20. Données complémentaires sur les faibles lecteurs... op. cit. 


\section{Préambule. Scénarios « en chiasme »}

Dans la perspective qui est la nôtre, la question de fond posée par la faible lecture interroge plutôt le comment que le qui. Il importe moins d'éclaircir «qui est faible lecteur? " que de déchiffrer « comment le devient-on? ». La position de faible lecteur apparait plutôt comme le point d'une trajectoire complexe que comme le résultat de dispositions sociales déterminées et immuables. Pour cette raison, le faible lectorat ne constitue ni un habitus culturel systématisé ni une catégorie sociologique spécifique dotée de capital culturel et économique distinct. Bien que fortement représentée dans certaines catégories, les faibles lecteurs recouvrent une large partie de l'échelle sociale et des trajectoires culturelles, professionnelles, et géographiques distinctives. Tout au plus, le faible lectorat se caractérise, dans son rapport à la lecture, par un ensemble de conditions pratiques et sociales qui peuvent être commandées par un modèle structural ; c'est ce que nous tenterons d'explorer. Mais on devient faible lecteur en empruntant des trajectoires très variées et qui sont d'autant plus importantes qu'elles formulent l'articulation des conditions évolutives d'accès à la culture dominante. Autrement dit, «à un volume déterminé de capital (culturel) hérité, correspond un faisceau de trajectoires à peu près équiprobables conduisant à des positions à peu près équivalentes ${ }^{1}$ ». Les faibles lecteurs se retrouvent sur cette pratique lectorale spécifique armés de capital hérité variable, de "propriétés secondaires » également variables sexe, appartenance géographique, ethnique - mais surtout de positions sociales très diverses articulées dans l'origine sociale, la catégorie socioprofessionnelle et le niveau d'instruction ${ }^{2}$. 


\title{
Trajectoires sociales et scénarios de lecture : quelques cheminements
}

\author{
L'école et la lecture : liaisons passagères ${ }^{3}$
}

\section{Entretien 1}

2 Issue d'une famille d'ouvriers d'origine paysanne, Christiane, âgée de dix-neuf ans, est elle-même ouvrière dans une entreprise allemande frontalière et vit chez ses parents dans une petite commune d'Alsace. Elle a suivi des études secondaires dans un collège d'enseignement secondaire (CES) local d'où elle est sortie à l'âge de dix-sept ans sans avoir passé son baccalauréat et a obtenu, par la suite, un CAP. Pendant son enfance et son adolescence, Christiane a fréquenté les lectures contraignantes du cycle scolaire et quelques bandes dessinées, des best-sellers du roman historique (Paris au mois d'août) et des ouvrages de la littérature dite "à l'eau de rose» (Guy des Cars, Harlequin, etc.). Entrée à l'âge de dix-huit ans dans la vie professionnelle, elle affirme avoir alors momentanément réduit sensiblement ses lectures pendant quelques mois pour reprendre ensuite, à un rythme très espacé, des ouvrages de genres et de thématiques "ciblés»: livres sur le cyclisme, sur la drogue, romans historiques sur l'époque contemporaine. Christiane ne lit plus les auteurs de la littérature classique figurant au programme des enseignements secondaires. Elle écoute de temps à autre de la musique avec quelques amis, achète ses livres - très épisodiquement - à l'hypermarché le plus proche ou dans une petite librairie où elle achète aussi chaque semaine ses Paris-Match et autres magazines d'actualité, et détient un petit stock d'ouvrages dans sa chambre. Son passage à l'école apparaît comme une expérience accidentelle dans sa trajectoire de lectrice : le refus de lire les ouvrages imposés par les enseignants a fait la place à la stimulation de lire des ouvrages rendus publics par la télévision, ou qui ont peuplé des conversations amicales.

3 Si l'entrée dans l'univers professionnel de la classe ouvrière a correspondu pour Christiane à une réduction quantitative de ses lectures, elle s'est en revanche soldée par une certaine fidélité à des genres et thématiques promus plutôt par la culture de masse que par l'institution scolaire. Les références aux auteurs, qui structurent les ouvrages scolaires et la classification des livres selon la culture institutionnelle sont absentes des évocations de Christiane : elle cherche plus un thème, une expérience - le combat, les intrigues amoureuses, les acquisitions sportives, la drogue - que l'accumulation de savoir didactique sur la littérature et ses auteurs.

\section{Entretien $\mathbf{4 0}$}

4 Martine a dix-neuf ans, est fille d'un ouvrier et d'une employée, aime à peu près les mêmes lectures que Christiane - à y ajouter la bande dessinée - et vit chez ses parents. Elle est bachelière et commence une formation para-juridique courte. Les copains, la plage, la musique pop sont ses loisirs favoris, non pas la lecture. Au lycée elle a lu pour avoir son bac, les fascicules de préparation aux examens, ou les classiques destinés à des études spécifiques de textes en classe. Bien que d'un niveau d'instruction supérieur à celui de Christiane, elle ne lit plus ces textes à usage purement scolaire, selon elle, et consulte très rarement des bandes dessinées empruntées à des amis, des ouvrages sur la drogue ou des magazines consacrés à la jeunesse et à ses loisirs - 
musicaux essentiellement. Le stock privé collecté par son père ne semble pas pour l'instant encourager de plus larges lectures, pas plus que l'abonnement à France-Loisirs offert par une parente à l'occasion d'un anniversaire.

Lorsqu'elle visite ses grands-parents, en vacances, elle se trouve encore dans un milieu d'origine ouvrière, où elle ne dispose d'aucun contact avec le livre. La fin des études secondaires semble avoir pour elle marqué le renforcement de l'origine sociale et le retour à des pratiques lectorales conformes aux comportements culturels familiaux.

\section{Formation scolaire et formation professionnelle : les ruptures dans la trajectoire culturelle}

\section{Entretien 27}

6 Marie-Laure a vingt-sept ans, lit moins de dix livres par an, est horticultrice dans un village du midi de la France depuis qu'elle a quitté ses parents il y a quelques années ; ceux-ci sont artistes; elle-même a fait deux années d'études littéraires après le baccalauréat qui n'ont pas été sanctionnées par un diplôme.

7 Son discours est peuplé de références aux auteurs classiques et contemporains lus pendant l'adolescence familiale, et par la rupture sociale et culturelle qu'a constitué le "déracinement» géographique et familial: Marie-Laure a perdu ses références pratiques à la lecture légitime, et ne lit que de très rares bandes dessinées, un gros roman chaque été, et consulte partiellement des ouvrages consacrés à l'horticulture. L'essentiel de ses lectures régulières est tourné vers les magazines féminins. MarieLaure décrit son mince stock de livres - ouvrages sauvés ici et là dans le déménagement - avec la même connotation anarchique qu'elle perçoit dans ses cheminements géographiques.

\section{Entretien 36}

8 Issu d'une famille de la moyenne bourgeoisie, Marc, trente-neuf ans, est bachelier et représentant de commerce pour diverses entreprises. Il est célibataire, vit seul et rencontre quelques amis l'été ou pendant ses séances de bricolage dans la maison de campagne qu'il s'est construite lui-même. C'est à son père, collectionneur, qu'il emprunte les "grandes littératures » classiques et contemporaines, bien qu'il dispose d'une importante bibliothèque privée à son propre domicile. Marc lit beaucoup moins que jusqu'après son service militaire mais conserve néanmoins de ses acquis scolaires le goût pour la littérature romanesque et lit également des biographies, des ouvrages et des magazines d'actualité. Marc aime le sport et la musique classique, mais sa passion de jeunesse pour le cinéma n'atteint plus les sommets de la curiosité pour des ouvrages consacrés au septième art. L'image du père apparaît comme le socle d'appartenance à une catégorie sociale - bourgeoise et "cultivée » - dont Marc s'est marginalisé pour avoir changé de filière professionnelle par rapport aux tendances familiales. 


\section{Lecture en augmentation et évolution du statut familial}

\section{Entretien 38}

9 Marguerite a soixante-douze ans, elle est retraitée, a travaillé jusqu'à l'âge de soixante et un ans comme employée dactylo, est mère de six enfants. Son époux, artisanmenuisier, est décédé il y a huit ans et, après s'être dévouée pour ses tâches familiales pendant de très longues années où elle nous dit « ne pas avoir eu le temps de lire », elle exploite sa retraite au profit de loisirs comme les voyages et la lecture. Les enfants, employés ou artisans eux-mêmes, ont tous leur propre foyer. Les rencontres familiales assez fréquentes semblent faire office de stimulateurs de lectures pour Marguerite qui a très peu lu dans sa jeunesse et jusqu'après la fondation de son foyer; elle lit aujourd'hui quelques romans prêtés par sa fille ou des ouvrages et magazines pratiques à usage familial: condensés de droit, utiles pour la connaissance de formalités juridiques, ouvrages de médecine, magazines de mode où elle emprunte des idées de confection de vêtements pour ses enfants et petits-enfants, et des biographies de personnages publics de l'actualité contemporaine. Marguerite achète ses livres chez le libraire le plus proche qui lui vend aussi des magazines, mais elle ne courra pas toutes les librairies de Nice où elle habite pour trouver un ouvrage que la télévision aura promu.

\section{Entretien 9}

10 Jean-Luc a trente-sept ans, est marié et père d'une fillette de quatre ans, occupe un emploi de représentant de commerce et est titulaire d'un CAP de menuisier - son père est menuisier retraité et sa mère sans profession. Jean-Luc a très peu lu pendant son séjour au domicile de ses parents où les livres étaient absents. Depuis la création de son ménage et peut-être surtout depuis la naissance de sa fille, Jean-Luc achète progressivement des ouvrages et des encyclopédies, qu'il feuillette de temps à autre pour s'informer sur des questions de connaissances générales soulevées dans l'exercice de son métier. Il aime aussi les ouvrages ou articles consacrés à des questions scientifiques. Mais la littérature classique et contemporaine reconnue par la culture dominante est quasiment absente des préoccupations lectorales de Jean-Luc.

\section{Stabilité socioprofessionnelle et stabilité de la démarche de lecture}

\section{Entretien 42}

11 Mireille a cinquante-huit ans, vit en banlieue parisienne, n'a aucun diplôme, est mariée et mère de trois enfants âgés de plus de vingt ans, dont l'aîné est marié. Son époux est retraité de la RATP et ne lit aucun livre, mais consacre quelque temps chaque aprèsmidi à la lecture du journal. Mireille lit moins de quatre ouvrages par an, dont certains pourront être "dévorés » en quelques heures, mais ses lectures préférées sont celles des romans-photos et des magazines féminins. Sa fille, étudiante en institut universitaire de technologie (IUT), ne parvient pas à l'encourager à lire, mais ne lui prête jamais ses propres livres. Mireille ne connaît rien aux auteurs des ouvrages qu'elle lit, auxquels elle demande de "parler de la vie des gens", des artistes de variétés en particulier et de leurs amours. Elle lira aussi des best-sellers à thème d'actualité ou des textes à grand tirage adaptés à la télévision. Il n'y a pas de 
bibliothèque privée personnalisée pour Mireille à son domicile ; les enfants retiennent dans leur chambre les ouvrages dont ils ont l'usage. Mireille est originaire d'Algérie, et issue d'une famille de commerçants peu familiers du livre.

\section{Entretien 46}

12

Antoine est agriculteur, a cinquante et un ans et vit dans une petite commune de Bretagne avec son épouse et son fils. Il n'est titulaire que d'un ceritificat d'études primaires (CEP), n'a pratiquement jamais lu, ou très rarement si ce n'est pendant un séjour à l'hôpital. Et bien qu'actif dans le milieu syndical et associatif et cultivant un certain goût pour le théâtre amateur qu'il a longtemps pratiqué dans sa commune natale - où il vit aujourd'hui - il lit essentiellement des magazines consacrés au monde paysan et les quotidiens régionaux: ce qui l'intéresse particulièrement est composé de reportages sur des pays étrangers et de chroniques politiques. S'il peut citer quelques auteurs de littérature comme Malraux qui sont aussi des figures de l'histoire politique contemporaine, les classiques du roman lui sont quasiment étrangers.

\section{La lecture en hésitation}

\section{Entretien 2}

13 Jean-Pierre a cinquante-deux ans, vit dans une commune rurale de l'Oise avec sa femme et sa fille et enseigne le dessin industriel dans un établissement technique de la région. Jean-Pierre lit moins de dix livres par an dans lesquels il n'inclut pas les ouvrages consultés ponctuellement dans un but purement professionnel. Après avoir travaillé pendant plusieurs années comme ouvrier qualifié, il a repris ses études à l'âge de trente-deux ans pour l'obtention du diplôme du Conservatoire national des arts et métiers (CNAM). Son cheminement de lecteur pourrait être représenté par un graphisme « en dents de scie » : commençant par une faible carrière de lecteur après la sortie de l'école, il se remet à lire, et des ouvrages plus prestigieux - textes de psychologie, de sciences humaines et de littérature contemporaine - lorsqu'il reprend des études de technologie au CNAM. Mais aussitôt après la fin de ce cycle, le rythme initial de lectures ciblées et très espacées est retrouvé : ce sont des magazines consacrés à l'aéronautique, de très rares romans à grand tirage que Jean-Pierre lit parfois après avoir acheté ses ouvrages en réponse à une publicité reçue à domicile. Jean-Pierre dispose d'un très grand stock privé d'ouvrages accumulés depuis l'adolescence, mais affirme que sur ses choix de lecture, il est aujourd'hui « un peu plus indécis, parce qu'(il) ne sait pas dans quel domaine » se diriger, faute d'orientation livresque dans sa socialisation actuelle. A la première observation, une typologie de quatre scénarios apparaît dans cette diversité :

- scénario croissant: le faible lecteur ne lisait pas de livres jusqu'à l'émergence d'un changement d'ordre professionnel, éducatif, familial ou géographique qui lui a fait découvrir progressivement la lecture ;

- scénario décroissant : le faible lecteur a été un moyen ou un grand lecteur et a réduit ses lectures par le même type de modification de ses conditions sociales;

- scénario stable : le faible lecteur a toujours peu lu ; 
- enfin scénario variable : le faible lecteur a réduit ses lectures après avoir été un grand lecteur ayant eu accès au livre par un changement d'orientation professionnelle - ou d'implantation sociale et géographique - bien que provenant d'une pratique très faible de lecture. Il a peu lu à l'origine, a commencé à lire moyennement puis a réduit ensuite ses lectures pour revenir à l'intensité initiale.

14 En réalité ce qui nous apparaissait comme une typologie de scénarios n'est que l'expression formelle de trajectoires «en chiasme ${ }^{4}$ " qui recouvrent des ruptures d'ordre éducatif et culturel, socioprofessionnel, socioéconomique ou géographique, et qui s'ajustent sur la succession de tranches de vie.

15 Le discours de nos interlocuteurs manifeste bien cette structure, sur le plan narratif, lorsqu'il place les ruptures lectorales aux points saillants du cycle de vie, ceux liés à la formation scolaire, à la formation des ménages et à la trajectoire professionnelle.

«J'ai l'impression que c'est en relation avec une rupture d'équilibre. Dans le temps où j'étais au lycée, j'étais bien canalisée, je savais où j'étais, je savais ce qui me plaisait et j'arrivais à lire et puis après (...) j'ai eu des bouleversements dans ma vie, j'ai bougé, ma vie a changé, et c'est à partir de ce moment-là que j'ai commencé à beaucoup moins lire»

(E 27, horticultrice, travailleuse intermittente, 27 ans, bachelière).

La structure du discours E 25, employée dans une parfumerie, est plus «parlante » encore lorsqu'elle emprunte l'analogie entre l'expérience de vie personnelle et l'univers narratif des ouvrages et de leurs auteurs :

«Vian c'était en seconde, Vian et Camus, parce que tous les deux inspirent pas mal de malaises. J'ai lu L'écume des jours, L'étranger, un peu dans la même période, un peu après, J'irai cracher sur vos tombes, L'arrache-cœur, c'est après la dépression nerveuse de maman et je n'étais pas très bien. Le lycée ne me plaisait pas, donc j'ai beaucoup lu cette année-là, et surtout ces livres-là parce que l'ambiance du lycée c'était l'horreur. »

(E 25, employée dans une parfumerie, âgée de 20 ans, résidant dans une commune rurale de l'Oise).

\section{La faible lecture et la trajectoire scolaire}

Le cycle scolaire et ses ruptures apparaissent dans les cheminements des faibles lecteurs comme le cadre prédominant d'émergence des ruptures initiales dans les pratiques de lecture. La sortie du système scolaire en est le point saillant, qui marque la réduction des lectures surtout lorsqu'elle sanctionne une formation technique ou une expérience d'échec scolaire dans le cadre d'établissements d'enseignement secondaire. Les jeunes adultes issus de familles ouvrières, paysannes ou d'employés, et qui se trouvent en situation d'échec scolaire ou de substitution d'une formation technique ou professionnelle à la formation générale dispensée au lycée ou au collège, constituent de faibles lecteurs typiques des scénarios de lecture décroissante. Ainsi Christiane (E 1), dix-neuf ans, qui travaille comme ouvrière depuis quelques mois, a abandonné ses études secondaires à dix-sept ans pour préparer un CAP, en même temps que les rares lectures de la littérature classique imposées en classe, pour se tourner vers la bande dessinée, les ouvrages et magazines consacrés au sport et les textes traitant de l'histoire contemporaine. Assez proche, la trajectoire de Martine (E 40), du même âge est sensiblement différente puisqu'elle ne s'inscrit pas dans un processus d'échec scolaire. Pour autant, la fin du cycle secondaire et l'obtention du baccalauréat vont marquer pour elle la cessation des lectures à usage scolaire et la conservation des 
ouvrages consacrés à la drogue, des magazines de variétés et de musique pop et des bandes dessinées.

Les ruptures liées à la trajectoire scolaire dans les pratiques de lecture sont donc fréquemment l'effet d'un échec scolaire ou d'une réorientation professionnelle ramenant les jeunes adultes sur des secteurs plus conformes à leur origine sociale : secteurs tertiaire et secondaire.

Cependant, ce qui apparaît comme une réduction quantitative de lecture consiste, pour ces lecteurs d'origine ouvrière et paysanne ou de familles de petits employés ou commerçants, en un changement dans la sélection des genres. On passe alors en réalité d'une lecture de textes légitimés par la culture dominante dispensée à l'école, à la lecture de textes non reconnus comme littérature "cultivée " - textes et ouvrages pratiques, ouvrages aux thèmes familiers des moyens de communication de masse, magazines sportifs ou consacrés à la moto. Ce glissement, dans la sélection des livres, d'un genre légitime à un autre, conforme aux pratiques culturelles d'origine, est vécu comme une forme d'autonomie par rapport au système scolaire transmetteur des valeurs culturelles légitimes :

«Une fois que j'ai quitté l'école, j'ai lu les bouquins que j'avais envie de lire. »

(E 3, femme au foyer de quarante-cinq ans, époux enseignant, bachelière, sans emploi).

«Les livres que tu lis à l'école, tu n'as pas toujours envie de les lire, parce que tu

n'as pas toujours le droit de choisir les livres que tu as envie de lire.»

( $E$ 1, ouvrière, 19 ans, titulaire d'un CAP).

Plus évidente encore est la fonction réductrice de la sortie du système scolaire, lorsqu'elle se manifeste à la suite d'une seconde formation ou d'une formation tardive telle que celle suivie par cet enseignant de dessin industriel qui après avoir travaillé comme ouvrier qualifié a repris des études à l'âge de trente-deux ans ${ }^{5}$. Propulsé dans le milieu universitaire, il se met alors à la lecture d'ouvrages de sciences humaines et de littérature contemporaine qui peuplent la socialisation universitaire de l'époque (années 1960). La fin de cette formation tardive et l'entrée dans la vie professionnelle même si elle consacre l'insertion dans l'éducation nationale - marquera l'abandon de lectures diversifiées et le retour à des lectures plus ciblées, sur des intérêts exclusivement professionnels et techniques (revues d'information aéronautique).

Ces circonvolutions dans les trajectoires, bien que déterminées par l'origine sociale des lecteurs, n'en permettent pas moins d'accumuler un certain volume de capital culturel pendant la formation scolaire. Si on est faible lecteur, on est néanmoins capable de citer des auteurs-phares de la littérature contemporaine ou de celle du $\mathrm{xIX}^{\mathrm{e}}$ siècle (Saint-Exupéry, Aldous Huxley, Jules Verne, Balzac), dont l'étude balise les enseignements secondaires traditionnels.

Malgré cela, le faible lectorat se distingue plutôt par la faiblesse des acquis scolaires dans l'articulation des actes positifs et volontaires de lecture.

\section{Mobilité socioprofessionnelle et scénario de lecture}

La sortie de l'univers familial et domestique et la création d'un foyer constituent cette autre forme de rupture dans les trajectoires lectorales, souvent associée à la précédente, au demeurant, parce qu'elle s'accompagne aussi de mécanismes de mobilité géographique - plus ou moins marqués - et donc de modifications des conditions sociales et culturelles. On change de domicile et dans le déménagement se 
perdent certains livres et le rapport physique et matériel à la lecture. En réalité c'est la mobilité sociale qui se manifeste par ces ruptures dans les pratiques lectorales, apparemment liées à l'évolution de l'univers familial.

L'accroissement des lectures pourra ainsi accompagner des formes de mobilité ascendante tandis que l'évolution inverse de la pratique lectorale constituera l'effet de la mobilité descendante. Témoin cette horticultrice de vingt-sept ans qui, après avoir quitté le foyer de ses parents artistes, fait deux années d'études littéraires non sanctionnées par un diplôme, puis s'« exile » dans un petit village du midi de la France où elle travaille dans le milieu agricole et ne lit que de très rares bandes dessinées ou quelques magazines féminins ou ouvrages professionnels, et a abandonné les grands classiques de la littérature légitime :

«Les gens que je fréquente ici ne lisent pas, donc ils ne parlent jamais de bouquins,... ce dont ils parlent, c'est des BD, Le loup garou des îles..., non je ne suis pas dans un milieu qui me pousse à lire. » (E 27)

La réduction des lectures depuis la séparation d'avec le milieu familial et après le déracinement géographique et social sont interprétés par notre interlocutrice comme l'effet d'une perte d'identité sociale, et en référence constante à ce qui est présenté comme un capital culturel d'origine plus prestigieux, muséographié dans les vestiges de la bibliothèque parentale qui ont résisté au déménagement et qui sont éparpillés anarchiquement dans le logement. La perte de la socialisation familiale du livre et d'une partie des livres du foyer d'origine peut accompagner un processus de déqualification. Il est probable que ces mécanismes soient souvent caractéristiques des scénarios de lecture en réduction. On peut imaginer que la déqualification menant au chômage procède à des effets similaires en matière de pratique de lecture.

Autre effet de la conjonction entre mobilité socioprofessionnelle et mobilité culturelle, l'accroissement des lectures et du stock d'ouvrages possédés par les fils d'artisans ou d'employés accédés à des postes de cadres du secteur tertiaire, après la création d'un ménage : ainsi cet agent commercial de trente-sept ans, marié et père d'une petite fille, se dote progressivement des livres qu'il n'a jamais eus au foyer de ses parents, artisan et femme au foyer. L'investissement personnel sur le livre et la lecture est ici présenté comme l'effet du souci de s'inscrire dans un processus d'ascension sociale: une stratégie qui prend la forme d'une "politique du livre »; les ouvrages achetés sont presque tous stockés dans la chambre de l'enfant. (E 9).

Les ruptures qui marquent les pratiques de lecture sont fréquemment associées à la conjonction des biographies familiales et socioprofessionnelles et des biographies culturelles. Elles permettent ainsi à des lecteurs d'origines sociales variables de se retrouver sur des pratiques similaires. Ainsi des faibles lecteurs déqualifiés provenant de milieux bourgeois "cultivés" côtoieront des faibles lecteurs urbains d'origine paysanne et reconvertis dans l'artisanat, telle cette coiffeuse de cinquante-huit ans, résidant dans la banlieue de $\mathrm{Caen}^{6}$ et dont le fils aîné est chercheur dans une institution nationale. Après une longue période de très rares lectures, pendant l'enfance et après la création du ménage, elle entreprend une lecture progressive vers l'âge de quarante ans, au moment où ses enfants entament leurs études et injectent dans le foyer livres universitaires et sujets de conversation intellectuelle. Les mécanismes de mobilité sociale ascendante et d'élargissement du recrutement scolaire après la seconde guerre mondiale ont conduit à l'acquisition progressive dans les classes populaires, de volumes faibles de capital culturel, par la lecture en particulier. La mobilité culturelle a opéré 
dans le même sens, ascendant, que la mobilité sociale et économique, de même que dans les années 1970, elle s'est manifestée, en sens contraire dans les générations déqualifiées issues de la petite et de la moyenne bourgeoisie.

Mais si deux mouvements contraires aboutissent aux mêmes pratiques lectorales de faible envergure, la configuration du capital culturel s'y conclut différemment: le lecteur qui est en scénario croissant de lecture ne dispose pas des références à la lecture dominante - citations d'auteurs légitimes, classification par genres littéraires - que conservent les lecteurs en scénarios de lecture réduite, du fait du haut niveau de leur capital culturel d'origine. Ils utiliseront d'ailleurs ces références comme emblèmes identitaires: les auteurs et les titres prestigieux sont les grandes figures du mythe d'origine sociale qu'alimentent les faibles lecteurs issus des classes moyennes.

Ces scénarios « en chiasme » se retrouvent dans la rupture que constitue la cessation des activités professionnelles par le chômage ou par la retraite. Traditionnellement le discours rationalise cette dernière en la présentant comme une période de temps libre et de loisirs par excellence ${ }^{7}$, opposée au temps contraint et au « manque de temps pour la lecture » qui caractérise la représentation de la période active.

De fait, la retraite ne peut encourager la lecture que si celle-ci dispose de réseaux de socialisation et de supports de communication que le lecteur aura constitués pendant la période active.

31 La retraite n'est pas nécessairement cette période où l'on accumule systématiquement des activités de loisirs; elle s'inscrit dans les formes de socialisation tissées dans la vie active, même si le discours la perçoit en opposition totale avec les activités professionnelles. De fait si cette opposition se manifeste, ce sera plutôt dans le sens de la réduction des activités de loisirs qui nécessitent des supports vigoureux de socialisation. Dans bien des cas, la perte de liens sociaux qui se manifeste au moment de la retraite correspond en réalité à la réduction des loisirs soutenus par ces liens. On observera le plus souvent des ruptures réductrices des pratiques de lecture au début de la retraite. A moins que ces pratiques ne se socialisent dans le cadre familial, lieu de repli post-professionnel, comme on peut le constater dans la trajectoire de cette secrétaire retraitée, âgée de soixante-douze ans, ayant pratiqué une dévotion zélée à l'égard de ses tâches d'épouse et de mère jusqu'à l'âge de soixante et un ans, et qui après le décès de son époux se met à lire faiblement sur l'encouragement de ses enfants, désormais mariés et insérés professionnellement ${ }^{8}$. L'accès à la lecture est donc ici le produit des structures évolutives de la famille plutôt que l'effet du cycle de vie.

Dans le même ordre d'idées, la rupture que représente le chômage ne peut être interprétée au plan sociologique qu'en référence au volume du capital culturel accumulé et de l'origine sociale. Le "temps libre» n'est rien d'autre que du temps social disponible, on ne lit pas plus pendant la période du chômage, simplement parce qu'elle constitue une période inactive. Le plus souvent elle représente une perte de socialisation et de liens sociaux encourageant la lecture. C'est le cas des jeunes adultes récemment sortis du système scolaire et à la recherche d'un premier emploi, de lecteurs dont le milieu professionnel constituait un réseau de stimulation à la lecture, ou de chômeurs en situation de déqualification. 


\section{«Accidents » de parcours lectoraux}

Le discours qui structure l'évocation des trajectoires des faibles lecteurs, comporte un effet récurrent : les ruptures (réduction ou accroissement des lectures) y prennent fréquemment la forme événementielle d'un séjour à l'hôpital ou d'un événement physiologique: tout se passe comme si la rupture dans les pratiques lectorales constituait d'abord la réaction incorporée à l'égard de certains enjeux sociologiques. L'illusion événementielle qui s'infiltre dans l'évocation de l'autobiographie du lecteur est la formulation de l'approche « accidentaliste » de la pratique. Ainsi cet agriculteur de cinquante-quatre ans, non lecteur de livres, nous explique qu'il a connu une courte période de lectures pendant un séjour à l'hôpital, par l'effet du don de livres que cet événement suggère.

La « dépression nerveuse " suivie d'une hospitalisation constitue une autre formulation de la rupture dans l'évolution de la lecture, et en particulier chez certaines femmes mariées ayant déjà constitué un foyer et connu des formes multiples de mobilité professionnelle.

L'hôpital réapparaît ainsi souvent comme le lieu de stimulation de la lecture, comme si les lecteurs formulaient de cette manière la faiblesse de leur pratique en la mettant en scène dans un cadre pratique transitoire, thérapeutique et clos; tout se passe comme si on voulait ainsi signifier l'éloignement de la lecture et du livre par rapport au milieu matériel et social habituel des lecteurs. Certains ont vécu cette rupture momentanée comme un simple accident de parcours: les seules lectures effectuées pendant les dernières années l'ont été pendant le séjour à l'hôpital. D'autres ont retenu cette rupture comme le point de départ d'une nouvelle « carrière lectorale ».

De fait, cette formulation événementielle et accidentelle de la rupture dans le scénario de lecture met en évidence la rupture qui l'accompagne dans les conditions socioéconomiques des lecteurs: la croissance ou la décroissance des lectures est le résultat quasi systématique de la mobilité, qu'elle soit sociale, géographique ou économique, et de la divergence entre la position socioéconomique et les acquis culturels, quelles que soient les modalités de cette divergence. Les scénarios stables le montrent bien qui caractérisent des situations sociales plus ou moins marquées par la stabilité : la reproduction sociale de la classe, de la catégorie socioprofessionnelle et la stabilité géographique semblent contribuer chez les faibles lecteurs, à une certaine stabilité des dispositions culturelles et en particulier des pratiques de la lecture et du livre. Ces faibles lecteurs «stables » étant le plus souvent dans les échelons inférieurs de l'échelle sociale, ces scénarios apparaissent comme la résultante d'une certaine conformité entre la position socioéconomique et les acquis culturels, quelles que soient les modalités de cette convergence.

Il reste à déchiffrer la dialectique de ces divergences et convergences qui tendent à regrouper des scénarios complexes sur une pratique commune dotée de sa logique propre. 


\section{NOTES}

1. BOURDIEU Pierre. La Distinction, op. cit., p. 122.

2. Cette variable est, comme nous l'avons précisé p. 9, beaucoup plus spécifique de ce rapport à la lecture.

3. Les prénoms et patronymes mentionnés sont purement fictifs. Les indications numériques précédées de la lettre $\mathrm{E}$ renvoient à la numérotation des entretiens dont la liste est détaillée en annexes, p. 106 et suivantes.

4. Nous nous référons à la terminologie de la "structure en chiasme » au moyen de laquelle Pierre BOURDIEU analyse les mécanismes de la classe construite cf. La Distinction, op. cit. p. 130.

5. Voir annexes, p. 114 entretien $n^{\circ} 2$.

6. Voir annexes, p. 112 et suivantes entretien $n^{\circ} 17$.

7. Ibid.

8. Voir annexes, p. 120 et suivantes entretien $n^{\circ} 38$. 


\section{Première partie. Le livre en pratiques}

1 Lorsque l'on admet qu'il existe chez les faibles lecteurs des scénarios variables de lecture impliqués dans un faisceau de trajectoires sociales et culturelles, on est tenté de négliger ce qui constitue pourtant une logique structurale dans les pratiques ellesmêmes, que la fréquentation répétée des matériaux d'enquêtes finit par faire émerger. Cette logique se manifeste non seulement par la spécificité des cadres sociaux qui soutiennent la circulation et la lecture des livres, mais aussi par la sélectivité de la pratique à l'égard des genres de livres, et par l'emploi du temps de la lecture.

2 Ce qui sera évoqué ici, plutôt que le rapport au livre tel qu'il se manifeste dans un groupe social que ne constituent pas les faibles lecteurs, c'est la logique des actes et des représentations qui régit une pratique de lecture dont l'homogénéité se situe dans son expression quantitative. C'est plus de la logique des actes que de celle des lecteurs en actes qu'il s'agira ici.

\section{L'information sur les livres et l'acquisition des livres}

3 Le cheminement suivi par un lecteur pour accéder au livre est une trajectoire sociale qui l'inscrit dans un faisceau de réseaux sociaux. L'acquisition d'un livre, quelles que soient ses modalités, suppose une connaissance minimale de l'ouvrage qui permet au lecteur de décoder son contenu, de le classifier, de lui attribuer un genre.

4 Les informations que les lecteurs détiennent sur les livres constituent des repères d'identification sociale. Un intellectuel parisien exerçant une profession académique, bénéficie de réseaux d'information sur le livre auxquels ne peut accéder un employé de banque résidant en province. Les lieux d'acquisition du livre sont donc significatifs de l'information dont disposent les acheteurs sur les livres : acquisition et connaissance du livre sont indissociables; nous les avons à dessein regroupées.

5 Les réseaux informatifs qui soutiennent les actes de faible lecture, quelle que soit la trajectoire professionnelle, familiale, éducative ou géographique des lecteurs, se regroupent autour de deux pôles, l'un replié sur le domaine privé le plus intime, l'autre ouvert sur le domaine public le plus anonyme : c'est entre la famille et les moyens de 
communication de masse qu'est diffusée l'information sur les livres et sur la lecture, bien que les modalités de cette dualité varient en fonction des trajectoires socioprofessionnelles et éducatives. Entre ces deux pôles, figurent différents réseaux de socialisation, professionnels, associatifs ou locaux ainsi que les réseaux de diffusion de l'information par correspondance et les réseaux commerciaux :

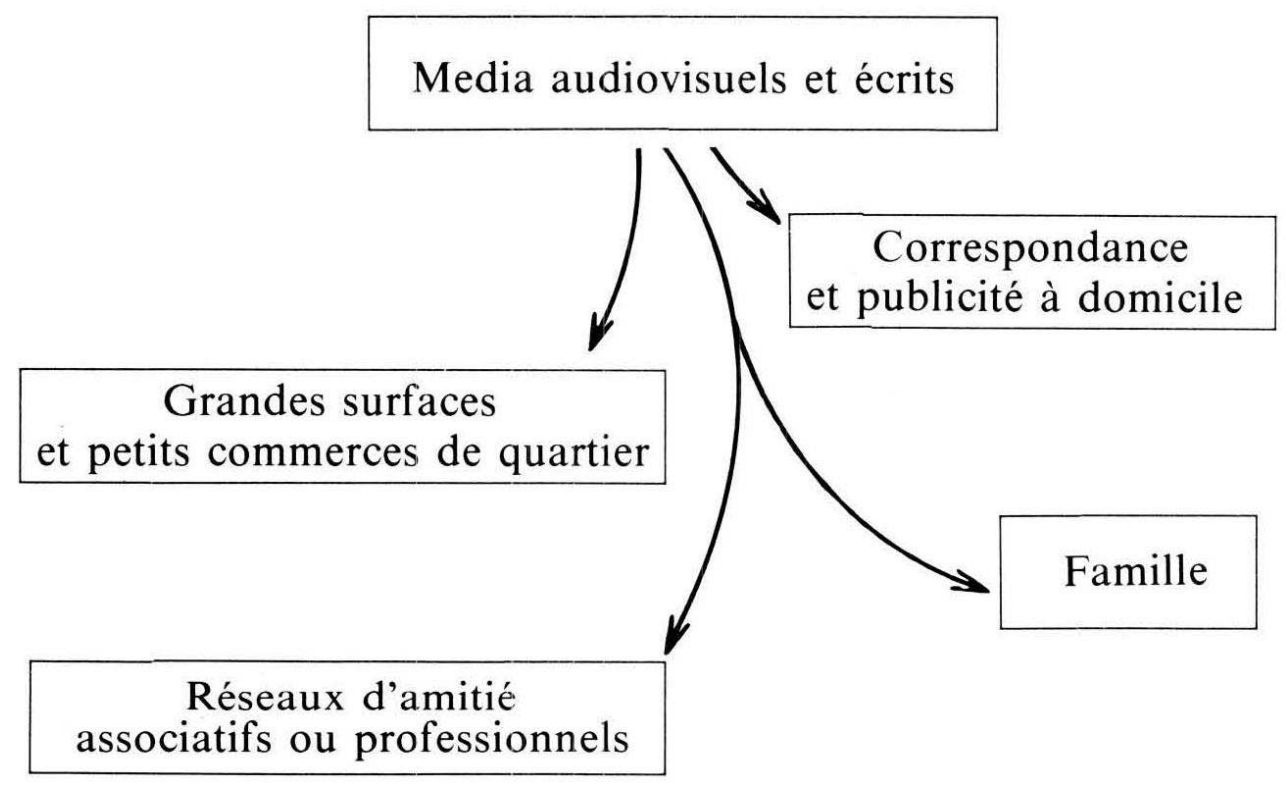

Tableau 1 : Les réseaux d'information et d'acquisition

6 Certains réseaux informatifs sur le livre brillent par leur absence dans ce faisceau. L'école est l'un d'entre eux, même pour ceux qui sont récemment issus du système scolaire. Si l'école diffuse des informations sur le livre, celles-ci sont rarement capitalisées par nos faibles lecteurs, parce qu'à la sortie de l'école, les références acquises dans la contrainte sont substituées à d'autres, bâties par les réseaux de la classe d'âge ou de la classe sociale. C'est le cas surtout des adolescents issus de l'enseignement technique et d'origine sociale peu élevée - milieux ouvriers, de petits artisans ou d'employés - et qui retrouvent des formes d'information plus conformes aux valeurs de leur groupe d'âge et de leur classe sociale.

7 Ici la faible lecture semble signifier l'une de ses spécificités : les informations acquises à l'école ne stimulent pas la lecture; elles permettent l'accumulation sommaire de connaissances - noms d'auteurs ou titres tout au plus - mais jamais la constitution d'une connaissance conduisant au choix (contenu, style, thématique du livre). Même les lecteurs d'origine sociale plus élevée et plus proches des valeurs de la culture dominante, donc ceux qui sont généralement en scénario de lecture décroissante, ont très rarement recours aux connaissances acquises à l'école ; tout au plus pourront-ils relire des ouvrages conservés depuis leur adolescence, mais ces lectures s'affirmeront rarement comme des découvertes menant à l'ouverture sur de nouveaux horizons littéraires.

Il faut ici rappeler que d'une manière générale ce faible lectorat dispose d'un acquis scolaire ne dépassant que très rarement le niveau du BEPC, ou du baccalauréat dans le meilleur des cas $^{1}$ : l'aspect limité des niveaux d'instruction révèle celui du poids de l'école et du système scolaire dans l'information qui soutient des pratiques de lecture de faible envergure. 
9 Ce qui vaut pour l'école vaut aussi pour le réseau des bibliothèques publiques, qu'elles soient municipales ou scolaires, et pour les bibliothèques d'entreprise. On peut donc se demander si la politique du ministère de la Culture en faveur de l'accroissement du réseau des bibliothèques publiques peut réellement encourager la lecture chez les faibles lecteurs.

10 L'impact de la bibliothèque se manifeste très faiblement dans les scénarios de lecture $\mathrm{y}$ compris pour d'anciens moyens lecteurs qui ont réduit leur rapport au livre, et qui ont fréquenté la bibliothèque dans le passé ; celle-ci s'estompe progressivement en même temps que le nombre de livres lus est réduit. La fréquentation de la bibliothèque est proportionnelle au nombre de livres lus : plus celui-ci est élevé, plus la bibliothèque est fréquentée, elle sera abandonnée en même temps que le livre. La bibliothèque apparaît dans les discours comme l'incarnation institutionnelle du livre: elle est la lecture institutionnalisée. Comme l'a montré Nicole Robine dans son étude sur Les jeunes travailleurs et la lecture ${ }^{2}$ la bibliothèque est perçue par le milieu étudié comme un cadre contraignant de lecture. Elle n'est fréquentée ni pour la lecture sur place ni pour l'emprunt d'ouvrages, pratique que l'on observe cependant chez des moyens lecteurs d'origine sociale peu élevée ${ }^{3}$.

11 Il nous semble que cette absence de la bibliothèque dans les scénarios de faible lecture doit être rapportée à trois types de spécificités de la pratique :

12 - La première a trait au volume et à la nature de l'information dont les lecteurs disposent sur les livres et la lecture légitimes. La bibliothèque s'en fait le vecteur, à condition d'en connaître le décodage : il faut savoir ce que l'on veut lire, avoir déjà opéré une sélection pour utiliser les services de la bibliothèque, ou du moins est-elle ainsi perçue. La faible lecture apparaît nettement de ce point de vue, et dans sa distinction, comme opposée à la lecture institutionnalisée qui se pratique dans les bibliothèques publiques.

13 - La seconde est relative à la forme pratique de la lecture chez les faibles lecteurs, dont la tendance manifeste est la non planification, la non structuration du choix et de la lecture de livres, qui ne peut s'accommoder du caractère contraignant des délais de remise des ouvrages et des sanctions encourues en cas de retard: la bibliothèque est presque perçue comme un inquisiteur de la lecture, par conséquent on la fuit. Elle ne constitue ni un lieu d'information ni un lieu d'acquisition d'ouvrages. Cette coiffeuse formule la désertion de la bibliothèque en termes de temps, qui expriment bien cette nécessité de planifier l'accès à la bibliothèque :

"J'allais à la bibliothèque quand j'étais dans une petite commune de l'Eure, j'étais abonnée... et puis ici à Caen, je ne l'ai pas fait, peut-être parce que (...) il faudrait disposer de temps (...). Je crois que c'est un entraînement, quand vous avez pris un engagement quelque part, ça vous oblige à continuer. »

(E 17)

14 - La troisième formulation de la désertion des bibliothèques publiques est dans le rapport matériel au livre-objet, dans l'appropriation du livre que ne permet pas l'emprunt systématisé en bibliothèque perçu comme "impersonnel»; et parce que l'appropriation matérielle du livre est très marquée chez les faibles lecteurs, la valeur du livre-objet est incompatible avec l'emprunt public d'un ouvrage qui circule entre de nombreuses mains.

Enfin, dernière grande absente de ce réseau informatif, la librairie "d'assortiment général ${ }^{4}$ » est rarement fréquentée; elle n'est ni un lieu d'acquisition ni un lieu 
d'information sur les livres. Les promenades exploratoires dans les librairies, auxquelles se prêtent certains moyens et grands lecteurs, sont inconnues des faibles lecteurs : dans ces sanctuaires du livre se pratique un culte dont les opérations ne sont pas dans la familiarité des faibles lecteurs, ni dans leurs habitudes culturelles, y compris pour ceux qui proviennent de classes proches de la culture dominante: les pratiques qui perdent leur support social perdent aussi leur raison d'être.

\section{Les médias et le livre}

L'illusion de l'intimisme de la lecture privée oppose traditionnellement cette dernière à la fréquentation de moyens de communication de masse. Il est vrai que la faible lecture de livres n'est pas l'auxiliaire de la lecture de la presse - quotidienne surtout : à deux types différents de supports correspondent des fonctions variables quant à l'acquisition du savoir. C'est la raison pour laquelle la presse écrite ne constitue que très rarement un support d'information sur les livres lorsque ceux-ci sont peu lus. La faible lecture de livres n'est pas sollicitée par la fréquentation des rubriques littéraires, et moins que les publicités d'éditeurs qui peuvent figurer dans les quotidiens. Mais dans ce dernier cas, c'est le support publicitaire qui est concerné plus que le support-presse : la sélectivité en genres de la faible lecture s'accomode mieux de la sémantique publicitaire que de celle de l'article du critique littéraire. Il y a dans ce rapport d'exclusion entre les deux supports d'information, l'impact des médias audiovisuels qui quant à eux, occupent une place de choix dans les réseaux d'information sur le livre : apparaissent ainsi dans les scénarios de faible lecture les émissions littéraires telles qu'Apostrophes, les interviews télévisées d'écrivains lors des magazines ou journaux d'information, les adaptations télévisées d'œuvres littéraires ainsi que diverses émissions où le livre s'infiltre au hasard de la composition des plateaux: le livre mis en images et mis en scène est un support privilégié d'information sur le livre et un stimulateur de lecture du texte. On passe ainsi de l'écrit en images à l'écrit en texte. L'image parait l'élément dominant dans ce rapport auxiliaire entre les médias audiovisuels et la lecture, car la radio ne figure pas très haut dans ce palmarès.

17 La prédominance de la télévision dans l'information sur le livre apparaissait rapidement à l'enquêteur qui voyait défiler dans ses entretiens un stock de titres récurrents de livres à gros tirages ayant constitué des événements télévisés notoires à leur parution : Les oiseaux se cachent pour mourir, Paris brûle-t-il et Le mal français étaient ainsi constamment et spontanément cités, à côté des prix littéraires qui occupent l'actualité médiatique audio-visuelle d'automne.

18 La télévision n'est donc pas la concurrente systématique de la lecture; on pourrait même se demander si elle n'en est pas parfois l'auxiliaire pour des pratiques de lecture de faible envergure. Peut-on pour autant en déduire la faiblesse de ces lectures dans leur rapport à l'écrit? Existe-t-il chez les faibles lecteurs une faiblesse du déchiffrement psychotechnique du texte qu'ils compenseraient en se soutenant par le recours à l'image ${ }^{5}$ ? Il nous semble qu'il convient ici de croiser ces mécanismes d'information sur les livres avec la fréquentation générale de la télévision par les faibles lecteurs. Les Pratiques culturelles des Français montrent en effet que les téléspectateurs les plus assidus se trouvent parmi les détenteurs de niveaux faibles d'instruction $^{6}$, majoritaires chez les faibles lecteurs. C'est donc par les moyens audiovisuels de communication que ces catégories sociales, milieux des classes 
populaires, accèdent à la culture et au livre en particulier. C'est par les réseaux spécifiques de la communication de masse que la connaissance du livre est socialisée. La communication audiovisuelle est l'un de ces réseaux d'information spécifiques. La faible lecture est donc stimulée par l'image non pas parce que celle-ci est un auxiliaire $\mathrm{du}$ déchiffrement du texte, mais parce qu'elle est l'un des vecteurs spécifiques de communication chez les faibles lecteurs; elle ne les empêchera pas de dévorer en quelques heures des ouvrages mis en scène à la télévision. La communication sur le livre s'articule dans les réseaux familiers de communication utilisés par les lecteurs, ceux qui par ailleurs peuplent les conversations de voisinage, ceux établis sur le lieu du travail ou en famille. Le livre est familiarisé par les moyens familiers de la communication et de l'interaction sociale.

\section{Achat par correspondance et publicité à domicile : le livre en promotion}

19 Nous nous trouvons ici dans un réseau d'acquisition du livre qui fait aussi et d'abord office de réseau de transmission d'information sur le livre. Le lecteur est sollicité dans les réseaux où il se trouve le plus fréquemment. Parmi ces réseaux est celui de l'achat à distance ou de l'achat en promotion.

Un représentant de commerce, 37 ans, titulaire d'un CAP de menuiserie s'équipe d'une encyclopédie et de quelques ouvrages, en promotion dans une publicité à domicile, au moment où il fonde un foyer et compose son aménagement domestique (E 9). Une étudiante de 19 ans, bachelière et lectrice sélective de bandes dessinées ou d'ouvrage sur la drogue, a reçu en cadeau lors de l'un de ses anniversaires un abonnement à France-Loisirs qu'elle méprise aujourd'hui et dont elle lit rarement les ouvrages reçus (E 40).

21 Le livre est ici un produit de consommation. On peut l'acheter comme un mobilier qu'on commande. Les achats par correspondance ou à la suite de la réception à domicile d'une publicité font partie de ces réseaux où le livre est pur produit. Ils sont rarement sollicités à l'initiative du lecteur, c'est eux qui viennent à lui, ils ne font pas l'objet d'une planification de lecture spécifique. On nous dira pour cette raison, et parce qu'ils dépersonnalisent le rapport à la lecture, que les livres acquis par ces réseaux ne sont pas systématiquement lus ; ils sont souvent réduits à leur pure matérialité, parce qu'ils sont joliment reliés ou brochés, s'inscrivent dans une série ou une collection «Club du Livre» par exemple. Ainsi si l'achat par correspondance stimule l'acquisition des livres, parce qu'il s'inscrit dans le modèle d'une pratique de consommation visant à réduire les budgets investis, il a tendance à restreindre le rapport au livre au seul registre de la matérialité. Le caractère contraignant et dépersonnalisé du choix d'un ouvrage inclus dans une série renforce cette image du livre consommé. L'achat par correspondance est ainsi souvent lié à la constitution d'un foyer et à l'aménagement du domicile : on s'abonne à France-Loisirs ou à quelque autre club du livre (ou on souscrit à l'achat d'une encyclopédie) comme on achète du mobilier; l'achat de meubles de séjour s'accompagne d'ailleurs de l'achat de ces collections. Les cadeaux d'anniversaire aux adolescents en cours de formation scolaire sont constitués d'abonnements ou de souscriptions à un achat de livre par correspondance. Mais ils cessent progressivement avec la maturation du ménage et des enfants. L'achat du livre en promotion s'inscrit donc dans un système global de répartition du budget qui, de ce fait, minore sa valeur 
symbolique d'objet culturel. D'une certaine manière, en accentuant la fonction de capital culturel objectivé du livre ${ }^{7}$ - «supports matériels» - l'achat par correspondance qui dépersonnalise le livre porte atteinte à sa fonction de «capital culturel incorporé » - «dispositions durables de l'organisme ». L'achat du livre par correspondance résulte de l'exclusion de celui-ci du rapport volontaire à la lecture.

\section{Les grandes surfaces et les petits commerces locaux}

L'achat dans les grandes surfaces relève du mécanisme socioéconomique que l'on observe dans les achats par correspondance. Les faibles lecteurs acquièrent le livre là où ils regroupent leurs pratiques générales de consommation. Mais si leur choix des livres est réglementé par les informations reçues auparavant dans les médias, l'achat dans les grandes surfaces relève néanmoins d'une démarche positive, qu'il n'est pas lorsqu'il s'effectue par correspondance. En outre, lorsqu'il trouve au supermarché un livre dont il a entendu parler à la télévision, le lecteur dispose d'un critère supplémentaire de sélection et d'information sur le livre. Il sait qu'alors ce livre-ci s'intègre à ses pratiques sociales et culturelles habituelles; si le livre est au supermarché, c'est qu'il lui est destiné.

L'achat dans la librairie-papeterie-kiosque du quartier relève d'un mécanisme social différent, dans la mesure où il sort des modes publics et anonymes de socialisation du livre pour s'inscrire dans les réseaux plus privés et personnalisés des échanges locaux. Il relève aussi plus manifestement d'un choix de lecture, même si, comme dans les petites communes ou dans les zones rurales, la librairie de quartier est l'unique lieu d'achat de livres.

24 Ainsi d'un côté l'achat dans la librairie-kiosque maintient le livre dans sa matérialité de produit de consommation acquis parmi d'autres produits, stylos, cahiers, etc. ${ }^{8}$. En même temps, le livre est acquis à la suite d'une demande spécifique faite par le lecteur à son libraire qui lui en parle, commande l'ouvrage s'il ne l'a pas. Ces achats se font donc le plus souvent en vue de la lecture. De ce fait, ils semblent plus pratiqués par les lecteurs en scénario de lecture croissante, tandis que les autres types de scénarios s'inscrivent dans la fréquentation plus marquée des grandes surfaces et de l'achat par correspondance. Le faible lecteur qui lit depuis peu trouve dans le rapport personnalisé avec son libraire-voisin, un support social et informatif de sa pratique lectorale. Il lui pose des questions sur le livre, la collection etc. l'opération de demande et de commande du livre dote ce type d'acquisition d'une forme volontaire qui particularise un livre spécifique (un titre, un thème, un auteur) tandis que l'achat par correspondance, c'est plutôt du livre qu'on achète qu'un livre particulier.

\section{Les amis, le réseau associatif et professionnel}

Avec la personnalisation et la privatisation progressive de l'acquisition des ouvrages, on change également de formes d'acquisition : ici elles donnent lieu à des échanges de livres et à des interactions sociales ciblées sur le réseau amical, le voisinage, le réseau associatif ou professionnel. L'emprunt ou le prêt appelle la lecture. C'est l'interaction spécifique d'un réseau social qui constitue le support d'information sur le livre : « un ami m'a parlé de ce livre et me l'a prêté »; " ma voisine m'a emprunté ce livre sur lequel nous avions bavardé »; «j'ai emprunté ce bouquin à un copain du syndicat après 
une réunion » etc. Le livre est ici socialisé dans les réseaux spécifiques d'interaction des lecteurs. Les jeunes et les femmes sont privilégiés par ce mécanisme: groupes de copains, de collègues de bureau, de voisines constituent des réseaux d'information et de circulation des ouvrages qui placent le livre dans la vie active et quotidienne, ce que ne suggèrent pas les représentations de la lecture envisagée comme acte de repli intime, d'isolement du monde. Le lecteur n'est jamais seul, quelles que soient les formes de lecture ; mais il n'y a pas là matière à spécifier la faible lecture par rapport à d'autres pratiques du livre. S'il existe une spécificité dans cette socialisation du livre, il faut la chercher dans la nature des réseaux, plus particuliers aux classes populaires et aux faibles niveaux d'instruction: les lieux de résidence (voisinage), les lieux du travail et les réseaux syndicaux en particulier. On ajoutera que pour ce qui est des lieux du travail, la socialisation du livre n'est pas institutionnalisée, elle n'est pas soutenue par l'institution professionnelle ou n'a pas un objectif professionnel; elle se fait dans le cadre des relations interindividuelles. Ce ne sont pas des livres sur le système bancaire que se passent les employées de banque qui travaillent côte à côte, c'est en tant que femmes qu'elles lisent ensemble la collection des Cartland. Les employées au guichet n'échangeront pas de livres avec le chef de service qui lit le dernier Yourcenar ou Elie Wiesel: les échanges à l'intérieur des réseaux de socialisation sont conformes à la hiérarchie des statuts professionnels et sociaux : on échange des livres avec celui qui détient les mêmes acquis culturels, les mêmes dispositions sociales à la lecture, les mêmes formes de modélisation des genres.

\section{La famille et le livre}

Au pôle tout à fait privé des formes d'acquisition des livres et de l'information, la famille occupe, dans les scénarios de faible lecture, une place privilégiée, et de manière paradoxale, dans la mesure où de nombreux lecteurs - à l'exception de ceux issus de milieux bourgeois ou assez élevés dans l'échelle sociale - se plaignent de n'avoir pas eu de livres dans leur enfance ou d'avoir été gênés par l'attitude familiale défavorable à l'égard de la lecture. Il faut préciser qu'il s'agit là surtout de lecteurs de la génération des 40-59 ans et parmi eux, ceux originaires de familles ouvrières ou paysannes. On peut se demander si la présence plus importante de la famille dans la socialisation du livre ne constitue pas un effet de l'histoire de la scolarisation et de l'élévation du niveau scolaire après la seconde guerre mondiale. C'est ainsi que dans les familles ouvrières où les jeunes adolescents sont de très faibles lecteurs ou des lecteurs exclusifs de bandes dessinées, des mères ou des sœurs aînées achètent des livres pour leur fils ou frère et l'encouragent à lire. Il est donc intéressant de voir que là où les niveaux d'instruction sont faibles, la famille peut se substituer à l'école dans la socialisation du livre et de la lecture, mais il ne s'agit ni des mêmes livres ni des mêmes lectures. On observera également que cette lecture et ces livres caractéristiques des milieux populaires sont socialisés dans le cadre d'une institution qui fait office de valeur identitaire: le familialisme propre aux "goûts » populaires familiarise aussi le livre, lorsque celui-ci existe. Enfin, les acquisitions de livres au sein de la proche parenté, par des prêts et des emprunts entre germains a aussi une fonction économique : on préfère échanger des ouvrages qu'il n'est pas utile d'acheter, surtout si ceux-ci s'échangent entre des ménages différents, mère et fille mariée par exemple. L'échange de livres s'inscrit dans les échanges de biens et de services par lesquels la parenté proche constitue une unité économique, au-delà des limites du ménage conjugal. 
Pour les lecteurs en scénario de lecture décroissante, les fonctions de l'intervention de la famille dans l'acquisition des livres et dans l'information sur les livres sont sensiblement différentes. Ici on a affaire à des lecteurs provenant souvent de familles de la moyenne bourgeoisie et qui ont connu des processus de déqualification ou de mobilité socioprofessionnelle descendante. Pour ceux-là, le capital culturel acquis dans la famille d'origine - références intellectuelles, citations d'auteurs, familiarité avec les genres légitimes, stock de livres conservés - constitue un repérage distinctif, un résidu de socialisation de la lecture légitime, par l'information qui est diffusée sur le livre et par les emprunts effectués. Témoin ce cadre commercial de 39 ans, célibataire vivant à quelques kilomètres du domicile de ses parents, qui dispose d'une faible stimulation à la lecture dans son milieu professionnel, et qui la trouve en revanche auprès de ses amis pendant la période de vacances, ou auprès de son père, collectionneur passionné de livres anciens et de gravures (E 36).

Finalement, d'un scénario de lecture à un autre et d'une trajectoire sociale à une autre, la famille se retrouve toujours en position prédominante dans les réseaux privés d'acquisition du livre.

La prédominance féminine est très nette dans la circulation des ouvrages, et surtout dans les milieux populaires peu dotés en diplômes. L'interaction mère-fille y est plus présente que la relation entre le père et le fils. C'est donc surtout du côté des femmes que circulent les livres et également entre germains de même sexe : la stimulation de la lecture se fait difficilement entre le frère et la sœur, entre le père et la fille.

30 Au sein de la famille, le livre sépare les sexes. Il paraît définir des spécificités sexuelles dans les goûts pour le livre et la lecture. Il trace aussi des frontières dans l'univers domestique entre les ouvrages des parents (rangés dans le salon ou la chambre à coucher), les ouvrages des germains masculins, les ouvrages des filles et les ouvrages des garçons, etc. Chaque sexe stocke ses ouvrages dans son lieu distinctif d'intimité. La famille et l'unité domestique opèrent une classification matérielle des livres qui distingue les sexes, les générations et aussi l'alliance de la consanguinité : les époux s'empruntent rarement des ouvrages qui circulent plus volontiers parmi les consanguins.

Même et y compris à l'intérieur du domaine privé de la parenté, la socialisation du livre s'inscrit dans les classifications propres au réseau interactions qui la soutient.

\section{Les formes d'acquisition : l'appropriation matérielle du livre}

C'est essentiellement par achats, emprunts privés et cadeaux que les faibles lecteurs acquièrent leurs livres. Parmi ces formes d'acquisition, l'achat prédomine nettement sur l'emprunt, le cadeau étant encore plus rare. Le livre apparaît rarement en tant qu'objet de dons et de contre-dons chez les faibles lecteurs, et plus particulièrement dans les classes populaires, sauf peut-être s'agissant de cadeaux offerts lors des rites de passage de l'enfance et de l'adolescence (acquisition d'un diplôme, passage entre deux cycles scolaires, rites de puberté, anniversaires, etc.). Parce que les représentations du livre lui attribuent la valeur symbolique propre à la culture dominante, elles l'excluent en même temps des échanges spécifiques aux milieux exclus de la culture dominante.

Pourtant, il est frappant de constater que si le livre est rarement offert, l'acquisition à usage personnel est essentiellement pratiquée par l'achat. On aurait tort de voir dans cette tendance à l'achat chez les faibles lecteurs une contradiction de leurs origines 
sociales : on pourrait ainsi penser que la faiblesse de la lecture est le produit des seules structures socioéconomiques caractéristiques du faible lectorat, et que plus les statuts sont bas, plus les budgets du livre sont faibles. L'étroitesse de ces budgets en volume est en réalité le produit du rythme de l'acquisition des livres et non des formes d'acquisition, ou du volume monétaire imparti à chaque livre. C'est parce que que les faibles lecteurs achètent rarement des livres ( 2 ou 3 par an) et de manière anarchique et non planifiée que le prix n'est pas un critère de sélection des ouvrages. Pourtant, on peut s'interroger sur la fonction de l'achat par correspondance qui est l'acquisition de livres en promotion et à prix réduits. Il nous parait plutôt relever des habitudes de consommation caractéristiques des classes populaires : on achète le livre en promotion comme la soupe en sachets ou les boîtes de conserve. Le prix intervient dans l'achat du livre non pas comme volume monétaire imparti, mais en tant qu'inscrit dans une habitude de consommation. De ce fait, il n'est pas un critère de sélection des ouvrages : sur le rayon du supermarché, un prix littéraire sera acheté en promotion si c'est lui qui est désiré, mais on ne saurait lui substituer un guide de voyages meilleur marché. Le prix du livre ne saurait influer sur la sélection du livre lorsque le budget imparti est si faible. Nos interlocuteurs affirmeront ainsi que parce que ces achats sont très espacés et rares, ils ne peuvent que consister en une réelle capitalisation, un réel investissement. L'achat du livre consiste à le conserver, à le stocker et à l'exposer : les livres en démonstration dans les salons sont aussi emblématiques; ils font office de repérage social ou de revendication de distinction sociale.

Si l'achat domine les formes d'acquisition des livres, c'est donc qu'il concrétise une certaine attitude, peut-être spécifique, relative au rapport matérialisé avec le livre. Les faibles lecteurs interrogés déclarent apprécier la possession des livres, la proximité avec les ouvrages ${ }^{9}$, bref le rapport physique avec le livre. L'achat manifeste ce souci d'appropriation physique du livre, cette exigence du livre à soi. Certaines de nos interlocutrices affirmaient avoir acheté des livres après les avoir lus par emprunt à des proches amis ou parents. Le livre que l'on aimé lire doit figurer dans le stock personnel et personnalisé d'imprimés disposés en témoignages de portion de vie.

Pour certains cependant, la tendance à l'achat du livre consiste à privatiser le rapport au livre : on achète parce qu'on méprise l'emprunt, surtout lorsqu'il se pratique hors du milieu familial. L'achat qui renforce la personnalisation du livre tend à fermer sa socialisation, au moment de la lecture notamment. Ainsi la coiffeuse de Caen, faible lectrice en scénario croissant, affirme qu'elle n'aime pas trop prêter les livres parce qu'elle trouve que le livre est quelque chose de très personnel, c'est pour une question d'attache à ce livre, dit-elle. (E 17).

\section{Les critères de sélection des ouvrages}

Les lieux et les types d'information et d'acquisition des ouvrages sélectionnent déjà les livres, indiquent des formes de classification des imprimés et des lecteurs. La sélection des livres et les critères qui la commandent sont aussi des indicateurs des structures de socialisation du livre, de son inscription dans l'univers culturel des lecteurs et surtout des formes que peut prendre la lecture elle-même de l'ouvrage choisi. Nous n'évoquerons ici que les ouvrages choisis volontairement et spécifiquement désirés par les lecteurs ; ne seront pas concernées les acquisitions par correspondance. 
Les auteurs sont de rares stimulateurs de sélection des ouvrages, sauf s'il s'agit, comme Barbara Cartland, d'un sigle ou de la dénomination d'une collection. Seuls les lecteurs en scénarios de lecture décroissante affirment choisir le livre pour son auteur, lorsqu'il s'agit notamment d'auteurs prestigieux de la littérature légitime. Le repère " auteur » ne peut constituer une orientation que pour ceux qui en détiennent les règles de décodage : lecteurs issus de familles bien dotées en capital culturel.

Le prix littéraire pourra aussi constituer un critère de sélection des ouvrages. Dans la faible lecture, ce critère s'affirme par la prédominance de la télévision dans les filières d'information sur les livres. L'attribution des prix littéraires d'automne est devenue un événement médiatique cyclique; dès lors le prix littéraire, par ailleurs doté de la légitimité propre à la culture dominante pénètre progressivement les canaux de l'évaluation des lecteurs peu familiers de cette culture, par l'intermédiaire des moyens de communication de masse.

Outre ces critères mineurs de sélection relatifs à la fonction intellectuelle du livre, les critères majeurs de sélection qui commandent les scénarios de faible lecture, se dégagent essentiellement des repérages matériels et éditoriaux portés sur les ouvrages : faute d'information substantielle sur le livre, la nature du texte sera décodée par :

la sémantique du titre,

les informations fournies par la quatrième couverture,

le thème et le genre littéraire,

le volume,

la forme éditoriale.

C'est donc l'ouvrage lui-même qui fournit son information. Le titre, la quatrième de couverture et le thème ou le genre indiquent le contenu de l'ouvrage ; le volume et la forme éditoriale, que l'on désire les plus légers possibles ne sont pas seulement des repères matériels : les gros ouvrages imprimés en caractères fins ne sont pas censés être destinés à des faibles lecteurs; le volume du livre peut l'écarter des scénarios de faible lecture.

On retrouve dans les formes de sélection des ouvrages la réduction du livre à sa matérialité chez les faibles lecteurs, comme nous l'avions déjà enregistrée dans d'autres pratiques, et qui manifeste un rapport au livre en marge de la culture savante.

Les formes et les lieux d'acquisition du livre chez les faibles lecteurs ne font pas que témoigner de l'inscription de sa socialisation dans les réseaux habituels d'interaction sociale. Ils distinguent aussi les livres sur un plan fonctionnel, entre les livres seulement acquis, les livres acquis, stockés et lus, les livres acquis, lus, prêtés ou revendus. Ainsi les livres empruntés sont le plus souvent lus, l'étant dans des circonstances volontaristes et non contraignantes; les livres achetés par correspondance sont fréquemment réduits à une matérialité ostentatoire : à défaut d'accumulation de connaissances, le livre-objet permet au moins l'accumulation de " capital culturel objectivé ». Mais le rapport volontaire à la lecture et au livre passe nécessairement par un échange interindividuel. Au supermarché, cet aspect volontaire de l'acquisition du livre semble minoré par le fait qu'on ait tendance à y acheter ce qui s'y trouve exposé, tandis que dans les commerces locaux, on y cherche ce que l'on veut acheter, quitte à commander l'ouvrage qui ne s'y trouve pas. 

foyer. C'est le destin du livre après l'achat qui est alors concerné. Acquisition et lecture des livres ne s'inscrivent pas dans la même logique fonctionnelle.

\section{Livres possédés, livres stockés et rangés, livres liquidés}

\section{distinction fonctionnelle entre l'ouvrage que l'on acquiert et qui est confiné à sa forme} objectivée, et l'ouvrage que l'on veut lire. Cette opposition, entre ouvrages possédés et ouvrages lus nous informe sur la nature du rapport matériel au livre en tant que " capital culturel objectivé », et sur les usages sociaux de ce capital qui n'est qu'objet, dans certains cas. Cette différence sensible dans le rapport au livre, et qui est déjà mise en évidence dans l'enquête sur les Pratiques culturelles des Français, par la distinction entre livres lus et livres possédés ${ }^{10}$ indique que si certains non lecteurs peuvent posséder des livres chez eux, des lecteurs assidus peuvent aussi ne pas en avoir et fréquenter les bibliothèques publiques. C'est donc une forme différente de socialisation du livre et de la lecture que l'on repère dans la distinction entre livres lus et livres possédés. 11 ne suffit donc pas de savoir combien de livres chaque lecteur possède, mais bien comment il les a acquis et quel destin il leur réserve après les avoir acquis.

Nous avons vu que du fait de la tendance à l'appropriation et à la personnalisation des livres, la faible lecture a tendance à appeler la conservation des ouvrages achetés.

La revente ou la liquidation des ouvrages après la lecture est plutôt spécifique d'une lecture plus intensive de livres, où l'acquisition des ouvrages relève d'une véritable planification. Lorsque le budget imparti au livre est mince, il n'exige pas une « politique » de rééquilibrage par la circulation des ouvrages revendus ou donnés.

Néanmoins on peut constater que lorsqu'elle existe, la liquidation des ouvrages opère une sélection des livres par le genre, par l'auteur ou par la forme éditoriale: les Harlequin et autres romans sentimentaux et policiers, édités en collection de poche et qui disposent d'une valeur matérielle et culturelle très faible - genre et auteur "illégitimes ", esthétique minorée - ne sont pas conservés dans les stocks privés des lecteurs : la littérature « illégitime » est celle qui circule le plus volontiers; les ouvrages conservés et stockés manifestent un effet de classification des genres, des auteurs et des collections.

Une dominante s'impose dans l'appropriation matérielle des livres par les faibles lecteurs : les stocks privés d'ouvrages se font extrêmement rares chez les lecteurs en scénario stable de faible lecture, ceux qui proviennent en majorité de couches populaires, ouvrières et paysannes. Là, les rares livres sont achetés pour être lus et s'évaporent progressivement du champ visuel domestique après la lecture. Dans ces milieux, le livre n'est pas objectivé, parce qu'il est rarement soutenu par un niveau élevé d'instruction.

Ce n'est pas le cas dans les foyers de faibles lecteurs en scénario de lecture croissante ou décroissante. Ici on observe assez souvent l'existence d'une bibliothèque personnelle, placée en position ostentatoire dans la salle de séjour. Pour les scénarios décroissants, il s'agit de stocks accumulés de longue date, vestiges ou repères identitaires d'une phase de la biographie plus riche en références livresques. Les 
scénarios de lecture croissante encouragent les stockages de livres accumulés de longue date, et font une place plus grande dans les bibliothèques aux ouvrages de collection Club, de reliure uniforme; l'uniformité éditoriale donne au stock une profondeur historique par laquelle le faible lecteur s'identifie à un modèle dominant qui l'exclut.

De fait, l'existence et le volume des stocks privés s'inscrivent dans les biographies familiales, ils sont historicisés. Parce que la possession des livres est sexuée ou répartie selon les âges des membres du ménage, elle contribue à une certaine forme de migration des ouvrages qui procède de l'effet structural de la famille nucléaire. Lorsque les enfants se marient, ils emportent avec eux les livres de leur enfance et de leur adolescence: nous retrouvons ce mécanisme inscrit dans l'acquisition de livres de collection Club au moment de la création du ménage et de l'unité domestique : le livre s'implique dans des processus de transmission et de reproduction sociales.

\section{Les lieux de rangement : repérage et classification}

La représentation du livre est donc aussi inscrite dans le rapport matériel aux ouvrages. Dans l'univers domestique, le rangement des livres sélectionne et classifie les genres, les formes éditoriales et les usages sociaux du livre. Si la conversation sélectionne et classifie les genres, les auteurs et les collections, les formes et les lieux du rangement lui répondent en procédant au même tri: les ouvrages de genre et d'auteurs « illégitimes » sont stockés dans des lieux de rangement qui leur dénient toute valeur de conservation. Les lieux de rangement constituent en quelque sorte la mise en scène du rapport au livre.

Si les grands auteurs - classiques ou contemporains légitimes - sont rangés dans la bibliothèque du salon auprès des livres de collection reliés et des encyclopédies, les livres de poche, les genres mineurs ou la littérature non romanesque ou à usage professionnel, seront rangés à l'intérieur des placards, parfois enveloppés dans quelque emballage. On ne montre que les livres légitimes, tels qu'ils apparaissent dans les représentations (voir ci-dessous). Les stocks visibles de livres sont donc plutôt spécifiques des scénarios mobiles ou variables de faible lecture. Les stocks invisibles de livres exclus de la lecture «cultivée " caractérisent des scénarios stables de faible lecture ${ }^{11}$.

Chez les petits agriculteurs, on voit ainsi les ouvrages s'évader dans les greniers ou dans d'autres lieux de stockage non visibles, après la lecture. Le livre montré n'est capital distinctif que là où sa valeur est réduite à son support matériel.

Outre cet effet de légitimité du livre, le rangement manifeste aussi les formes de distinction des statuts familiaux et les formes de transmission familiale. Les livres rangés dans le salon sont rarement consultés par les enfants, ce sont les livres des adultes. Lorsqu'ils se trouvent dans la chambre des enfants, ils tendent à marquer une forme assez nette de mobilité sociale ascendante et aussi de personnalisation des ouvrages: le rangement des ouvrages dans les chambres à coucher distingue les générations mais aussi des positions sociales potentielles différenciées au sein de la famille. Ainsi cette femme au foyer de 58 ans ${ }^{12}$, non diplômée et issue d'une famille de petits commerçants, résidant en banlieue parisienne, se plaint de la réticence de sa fille, étudiante en IUT, à lui prêter des ouvrages qu'elle détient dans sa chambre, et le plus souvent d'ailleurs achetés par notre interlocutrice. Le rangement du livre est donc classificateur social : la distinction entre le rapport de la mère et celui de la fille au livre 
manifeste une distinction de niveau d'instruction, et non pas seulement de génération. Le passage entre les deux générations tend à marquer un processus de mobilité sociale.

Enfin, le rangement du livre ordonnance les sexes : les livres de la collection Harlequin, les magazines féminins, les biographies d'actrices sont rangés dans les chambres des filles, les bandes dessinées, magazines de sport ou consacrés à la moto, peuplent les chambres des garçons. Ici, règne une sorte de désordre presque ostensible dans le rangement des ouvrages, tandis que les livres des filles et de leurs mères sont systématiquement classés, ordonnés, selon les genres ou les auteurs.

Le classement systématique des livres dans les rayons procède d'une opération manifestement plus féminine, comme l'achat des ouvrages d'ailleurs, surtout lorsqu'il s'effectue dans le cadre des acquisitions cycliques de provisions domestiques.

Les scénarios de faible lecture caractéristiques des classes populaires manifestent clairement le dynamisme féminin dans la circulation et dans les formes d'appropriation matérielle du livre.

\section{Rangements autobiographiques}

63 Si les rangements des ouvrages manifestent à la fois la réduction du livre à sa matérialité dans les pratiques de faible lecture et la distinction entre littératures légitimes et illégitimes, ils témoignent aussi, chez les lecteurs qui, en scénario de lecture variable ou décroissante ont accumulé les ouvrages sur une longue période, de l'inscription du livre dans le cycle de vie. Les promenades ethnographiques des enquêteurs devant les rayons indiquent que l'accumulation des ouvrages procède de la succession des tranches de vie et ne s'accompagne pas du rangement des ouvrages selon les classifications « légitimes" de la culture savante selon le genre, l'auteur, la date de publication. La datation des ouvrages est l'une des manifestations de ces mécanismes : elle renvoie le lecteur à sa biographie, associe la lecture à une période de son existence. Un peu comme la madeleine de Proust, le livre est mémorial.

La mise en scène autobiographique du rangement des ouvrages sollicite parfois la relecture de certains livres en forme de renouvellement de l'expérience passée. De fait, elle manifeste l'association de la lecture et du livre avec l'expérience vécue. Les scénarios pratiques de la faible lecture le montreront clairement lorsqu'ils mettent en valeur la fonction "expérimentale $»^{13}$ de la lecture : nos interlocuteurs ne nous disentils pas, comme un leitmotiv, que leurs lectures préférées sont celles qui mettent en scène des « expériences vécues »?

\section{NOTES}

1. Cf. Données complémentaires sur les faibles lecteurs... op. cit.

2. ROBINE Nicole. Les jeunes travailleurs et la lecture. - Paris : Documentation française, 1984. pp. 105-112. 
3. Témoin, cet artisan-tailleur qui achète et lit de nombreux livres dont il emprunte une partie à la bibliothèque de son quartier ( $\mathrm{E} 41)$.

4. Cf. ROBINE Nicole, op. cit. 44

5. C'est ce que semble conclure Nicole ROBINE dans son rapport sur Les jeunes travailleurs et la lecture, op. cit., pp. 142-145.

6. Pratiques culturelles..., op. cit., p. 55.

7. BOURDIEU Pierre, Les trois états du capital culturel. Actes de la recherche en sicences sociales, $\mathrm{n}^{\circ} 30$, 1979, pp. 3-6.

8. Il peut aussi être demandé ou même simplement découvert à la suite de l'achat hebdomadaire de la presse, et perd de ce fait sa spécificité de support-livre.

9. On a vu que cette attitude contribue à la désertion des bibliothèques publiques où l'emprunt des ouvrages apparaît comme une forme de dépersonnalisation du rapport au livre.

10. Pratiques culturelles des Français, évolution 1973-1981, op. cit.. p. 65.

11. Ils rejoignent dans cette pratique des scénarios de lecture de moyens lecteurs exclusifs de littérature « illégitime " (romans sentimentaux).

12. Voir annexes, p. 116 entretien $n^{\circ} 42$.

13. Qui donne accès ou qui mène à l'expérience. 


\section{Deuxième partie. La lecture en pratiques}

Si les faibles lecteurs ne constituent pas une entité sociale, ni une population spécifique, et si la faible lecture ne peut être repérée comme un corpus de pratiques systématiques, parce qu'elle résulte, comme nous l'avons vu, de la rencontre de scénarios variables de pratiques lectorales, elle se manifeste pourtant par un ensemble d'actes dont la récurrence parait formuler un «modèle structural», une forme systématique que l'on peut repérer dans les modalités de ces pratiques : la fréquence, la durée et les moments de lecture, les lieux où l'on lit, les formes psycho-techniques de la lecture et les cadres sociaux dans lesquels elle s'inscrit nous fourniront un premier cadre d'analyse.

\section{La lecture, une question de temps}

2 L'enquêteur qui enregistre indéfiniment les complaintes des faibles lecteurs sur leur manque de temps pour la lecture ne peut s'empêcher d'y voir la rationalisation discursive d'un enjeu social qui dépasse largement le pur investissement en volume de temps.

3 Pour en saisir les supports sociaux, on peut néanmoins tenter d'appliquer à ce discours sur le temps de la lecture - ou sur la lecture et le temps - le déchiffrage positiviste du rythme et de la fréquence de la lecture, de la durée et des moments, des circonstances de la lecture.

\section{La fréquence de la faible lecture : la logique des temps morts}

4 Si l'on s'en tient aux catégories statistiques initiales dans lesquelles notre enquête a été organisée, lire faiblement, c'est lire peu de livres. Ce déterminisme quantitatif ne peut manquer de structurer le rythme et la fréquence de la lecture. On imagine nécessairement des rapports très espacés avec le livre. Nos interlocuteurs nous entrainent dans cette illusion en nous rappelant fréquemment que la distance qu'ils entretiennent avec le livre se manifeste par le caractère fortuit de leur lecture, soumise 
aux aléas de l'apparition d'intérêts personnels. C'est la logique du « je lis quand j'en ai envie ", n'importe quand, à n'importe quel moment, et qui manifeste l'aspect non systématique d'une pratique que le lecteur veut « libre » et sans entrave.

La distinction entre lecture de presse et lecture de livres s'impose ici. La première est plus soumise à la forme cyclique de la parution des journaux et des magazines, bien qu'elle puisse consister en une simple consultation occasionnelle de documents rencontrés chez des amis, chez le médecin ou lors d'une visite familiale ponctuelle.

6 Dans la faible lecture de livres, le rythme apparaît sensiblement différent. Dans le cadre délimité d'une période annuelle, il n'offre aucun caractère cyclique ou de régularité. La seule exception à cette règle est la lecture systématique des vacances, qui peut-être d'ailleurs l'unique période de lecture. Mais si l'on entend par «fréquence » le nombre et la périodicité de l'occurence de la lecture de livres dans l'année, lire faiblement consiste à consulter le livre en l'absence de toute planification temporelle :

«En une semaine, je peux tomber sur des choses qui m'intéressent et je lirais 10 livres comme ça (...) mais aussi bien dans les six mois je ne ferais rien... Je ne sais pas, c'est toujours le hasard, ça m'intéresse, je prends ça, ça ne m'intéresse pas, je laisse. ${ }^{1}$ "

7 C'est ainsi que la jeune ouvrière alsacienne justifie sa difficulté à quantifier précisément sa lecture de livres, par la forme occasionnelle qu'elle lui attribue, et à la question : « combien de livres lisez-vous dans l'année? », elle répond:

« 6 à 7, ça dépend, parfois j'en lis un en une semaine et d'autres fois je n'en lis plus pendant quatre mois. "

(E 1)

8 La faible lecture ne relève pas du registre de la systématisation. Elle ne suppose pas une démarche délibérée d'organisation. Cette fréquence non planifiée et quasi anarchique d'une lecture apparaissant comme occasionnelle, tout à fait circonstanciée, ne contribue pas nécessairement à exclure le livre du cadre matériel de vie : il y a des livres qui traînent volontiers sur les tables de chevet, qui sont lus partiellement et alternativement et dont la lecture s'éternise : c'est la lecture simultanée de plusieurs ouvrages indéfiniment ouverts. Elle paraît plus spécifique des scénarios de lecture croissante ou variable qui s'accompagnent de l'accumulation des ouvrages à titre ostentatoire, comme si l'hyperprésence matérielle du livre entendait compenser sa présence très épisodique dans l'univers intellectuel du lecteur.

9 L'aspect occasionnel et circonstancié de la faible lecture s'affirme plutôt comme la marginalité du livre par rapport au temps actif du lecteur, au temps positif. Parce que sa lecture est perçue essentiellement comme un loisir et une fuite par rapport au rythme de vie professionnel et actif, elle apparaît comme le « négatif » - au sens quasi photographique du terme - de l'emploi du temps structuré et planifié de la vie active ; la faiblesse de la lecture, plus que dans sa dimension quantitative, apparaît comme l'effet qualitatif de la marginalisation du livre par rapport à la vie programmée en action. La lecture est au passif, on ne peut lui accorder que les «temps morts » qui surgissent hors du temps programmé de la vie active :

«Je n'ai jamais vraiment le temps de lire, je suis toujours en train de courir. Quand je travaille, je n'ai pas le temps et le soir je suis tellement crevée que je m'endors (...). Et en vacances, comme tout le monde, je lis dans des moments de détente (...) dans les temps morts. Quant je travaille à Paris, je suis obligée de prendre le métro, je préfère avoir de la lecture. Mais enfin un bouquin peut me durer deux mois si je ne le lis que dans le métro pendant dix minutes par jour... ça me dure très 
longtemps un bouquin. »

(E 27, horticultrice, 27 ans, célibataire).

Parce qu'elle se situe dans l'ordre du passif, la faible lecture n'est pas systématisée et refuse toute socialisation planifiée du rapport au livre, telle que la bibliothèque publique, où le temps de la lecture est quadrillé par les délais de remise des ouvrages :

« Il y a un truc qui me bloque avec les bibliothèques, c'est d'avoir un délai ; dans la mesure où je sais que j'ai un délai, je ne peux pas terminer le livre en temps voulu et après je me sens tellement coupable d'avoir prolongé le délai que je ne peux pas les lire, donc je n'aime pas les bibliothèques. »

(E 27 ans, horticultrice)

\section{A lecture marginale, moments marginaux : la lecture en patience}

11 Parce que non programmée, la fréquence de la faible lecture induit la marginalité des moments de lecture par rapport à l'emploi du temps actif. Les moments de lecture semblent se situer à des points de clôture ou de jonction de cycles d'activités, avant le sommeil de la nuit, la lecture est soporifique :

«C'est intéressant, je n'arriverais plus à éteindre la lumière, il faut que je lise ou sinon je n'arrive pas à dormir. »

(Ouvrière, 19 ans, célibataire).

«Je lis principalement le soir, avant de m'endormir dans le lit, mais si je n'ai pas fait trop de sport ou d'activité physique, parce que dès que je prends un livre, j'ai tendance à m'endormir ; à mon avis, c'est pour ça que je lis peu. »

(E 29, enseignant, 38 ans, marié).

La lecture encadre et organise le sommeil à défaut d'être elle-même planifiée dans la vie active. Elle apparaît dans les marges de l'emploi du temps actif : dans le temps de vacances annuelles, après le déjeuner pour les retraités ou les femmes au foyer et avant d'entamer un nouveau cycle d'activités dans l'après-midi, pendant l'attente chez le médecin, le dentiste.

\section{Le temps classificateur de la lecture}

Plutôt que de considérer le manque de temps pour la lecture comme un manque objectif et quantitatif que les rationalisations évoquent, c'est au temps représenté et hiérarchisé de la lecture qu'il faut se référer: la lecture est faible parce qu'elle est placée par les lecteurs hors du temps plein et positif; mais elle n'a pas non plus, dans le temps résiduel et extérieur au cycle d'activités, le statut dont disposent d'autres loisirs comme le sport, les promenades et les réunions familiales.

Martine ( $\mathrm{E} 40$, étudiante, 19 ans) nous explique ainsi qu'à choisir entre lire et écouter des disques, elle préfère la seconde alternative. Martial, 21 ans, titulaire d'un CEP affirmera que le sport et la musique l'attireront plus que la lecture pendant les weekends. (E 28).

En réalité le temps consacré à tel ou tel loisir est considéré comme temps actif s'il s'inscrit dans la logique de la socialisation des lecteurs ; la faiblesse de la lecture parait se situer dans l'aspect non rentable, non profitable du temps qui lui est imparti. La lecture n'est pas considérée comme une activité d'accumulation de profit symbolique ou social ${ }^{2}$, elle ne peut faire l'objet d'une programmation de temps spécifique. La 
lecture est faible parce que non soutenue par des interactions qu'elle n'est pas en mesure de reproduire elle-même.

Le temps qui lui est imparti est un temps marginalisé par rapport a celui consacré à la reproduction des liens sociaux habituels.

Ce manque de «temps social» s'exprime dans la complainte de cette jeune horticultrice, ex-étudiante en lettres, issue d'une famille d'artistes et qui se plaint de ne disposer d'aucune stimulation sociale à la lecture, ni dans son emploi du temps professionnel, ni dans son réseau de socialisation amicale et locale (E 27). Dans la même logique, la coiffeuse de Caen nous explique clairement que sa sensation de «manque de temps » pour la lecture n'est fonction que de la position qu'elle attribue à celle-ci dans sa hiérarchie des loisirs.

"Il faudrait vraiment donner la priorité à la lecture, on l'a donnée au sport parce que ça nous était bénéfique au point de vue santé, malheureusement, on a un métier sédentaire (...). Je préfèrerais qu'on consacre du temps à la lecture plutôt qu'à la télévision, seulement comme on est pris par une vie professionnelle, le dimanche, je regarde la télévision en faisant mon repassage, je ne peux pas lire vous voyez, quand on peut lire, c'est vraiment du temps à disposer, ce n'est possible que comme ça.» (E 17, Coiffeuse, à Caen, 58 ans).

Ainsi cette répartition hiérarchisée du temps s'ajuste sur un classement social. Ne pas avoir le temps pour la lecture, ou placer la lecture dans un temps négatif ou temps mort, c'est affirmer son exclusion des réseaux sociaux habituels des lecteurs mais aussi de la logique de leurs « goûts ».

"C'est une personne qui ne lit jamais, elle ne tient jamais un livre dans ses mains. D'après elle, elle n'a pas beaucoup de temps. Moi, j'ai l'impression que c'est une affaire de goût. Peut-être qu'elle n'a pas beaucoup de goût à lire. »

(E 42, femme au foyer, non diplômée, 58 ans).

Il peut paraître paradoxal que la lecture soit placée hors du temps actif et de ses cadres sociaux lorsque l'acquisition du livre - achat ou emprunt - s'est faite dans les formes habituelles et familières d'interactions. Qu'advient-il au livre après l'achat ou l'emprunt pour qu'il sorte alors, au moment où il s'apprête à être lu, du temps planifié des activités journalières, qu'elles soient professionnelles, associatives, familiales ou locales? On peut probablement repérer l'une des spécificités de la logique de la faible lecture : l'aspect limité de l'intervention des liens sociaux habituels dans le soutien de la lecture elle-même. Nous verrons dans la suite de notre exposé comment se manifeste cette faiblesse du soutien social de la faible lecture, et notamment dans les échanges d'information sur les textes lus ou dans leur usage social.

Cette logique du temps classificateur classe aussi les genres et les auteurs en termes de volume de temps imparti aux uns et aux autres. Ainsi le temps de la faible lecture est celui, apparemment réduit, exigé par les genres et les auteurs mineurs de la littérature illégitime, tandis que les auteurs "difficiles » de la "grande littérature » sont écartés parce que le volume de temps qu'ils exigent ne peut être imparti dans l'emploi du temps de la faible lecture :

«On se rend compte qu'il y a beaucoup de livres qu'on n'a pas eu le loisir de parcourir parce qu'on n'a pas assez de temps à consacrer à la lecture... Le Mal français, c'est de Peyrefitte, il écrit très bien, mais je crois qu'il faudrait avoir beaucoup de temps pour pouvoir... il faudrait pouvoir lire à un moment déterminé. » (E 17, coiffeuse, ibid.). 
Il faudrait donc distinguer, dans le temps de la lecture, celui que l'on consacre aux genres et aux auteurs légitimes et celui que l'on consacre aux genres et auteurs de seconde zone. La lecture affirme ici sa faiblesse lorsque le temps qui lui est imparti sélectionne les textes non légitimes, les seuls pour lesquels le lecteur restera assis plusieurs heures juqu'à la terminaison de l'ouvrage, tandis que les textes légitimes (auteurs et genres dits "difficiles») sont censés exiger plus de temps de lecture. Le temps représenté de la faible lecture est un temps mineur comme les livres auxquels il est consacré. Le temps de la lecture n'est rien d'autre qu'un classificateur des livres, des pratiques et des lecteurs.

\section{La lecture, une question de genres} littéraires du texte. Le terme « genre » est rarement employé, si ce n'est en réponse à la opération de classification, au sens maussien du terme ${ }^{3}$, c'est-à-dire dans sa fonction sociale : les classifications des objets renvoient à un ordre social, et pour ce qui nous concerne, le livre apparait comme un objet classifié et classificateur social, comme l'a montré Patrick Parmentier dans son étude sur les lecteurs de bibliothèques ${ }^{4}$.

L'idée de classification appelle donc, concernant le genre littéraire, celle de "légitimité » culturelle. Le classement des livres selon le genre relève de la «taxonomie ${ }^{5}$ » légitimée par l'institution culturelle, qu'il s'agisse du ministère, de la bibliothèque ou de l'école.

La terminologie du genre suppose nécessairement des connaissances quant aux formes de distinction des catégories d'ouvrages. Elle implique la connaissance de ces catégories. Son emploi par l'enquêteur ne manque pas, lorsqu'il s'adresse à un faible lecteur qui se présente comme démuni des instruments de distinction des ouvrages ( «Je manque de références » expliquait l'ouvrière alsacienne), de provoquer des effets de légitimation des pratiques propres au lecteur ou bien à l'inverse, d'évitement de la terminologie "générique ». De fait, l'enquêteur qui citait les catégories énoncées par l'enquête institutionnelle du ministère de la Culture se voyait orienté vers des formes de classification différentielles et partiellement étrangères au classement institutionnel ${ }^{6}$.

Certaines catégories étaient rarement citées par les lecteurs, telles que la poésie, le livre d'art ou même la bande dessinée, et faisaient l'objet d'une sollicitation spécifique de la part de l'enquêteur. La catégorie «scientifique " porte à confusion: elle dérive entre les livres de sciences naturelles - sur les plantes et les animaux - et ceux consacrés à l'astrophysique. Rarement seront mentionnées les sciences humaines et parmi celles-ci, l'histoire fait l'objet d'une catégorie spécifique (comme dans la classification des Pratiques culturelles).

La classification par " genres » implique la reconnaissance de la légitimité de l'œuvre et constitue un mode d'emploi pour la lecture, un code de déchiffrement du texte. Elle informe sur les modes de socialisation du livre et sur la logique de la perception des textes. Elle pourra aussi orienter la compréhension de la lecture en tant qu'acte de déchiffrement psychotechnique.

Une première observation s'impose : la faible lecture est partiellement soutenue par le classement des livres en genres, entendus comme catégories de style et de structure 
question de l'enquêteur qui le sollicite. Du genre, le discours passe au contenu thématique et narratif du texte. On observe ainsi un glissement de la terminologie du « livre de » (poésie, histoire, art) à celle du « livre sur » (la médecine, le sport, la guerre, les animaux, etc.), ou bien à la catégorie inscrite dans l'univers narratif du texte :

Question:

«Pourrais-tu faire une typologie des genres?»

Réponse :

«Oui, alors le genre le plus intéressant c'est à vrai dire une certaine philosophie dans l'action; c'est le cas de Saint-Exupéry qui était quand même un homme d'action et il avait à travers de tout ça toute une philosophie. »

(E 2, enseignant de dessin industriel, 52 ans). les livres trouvent leurs critères de classement, lorsque ceux-ci s'inscrivent dans des pratiques de faible lecture. De ce point de vue, il semble que la faible lecture se présente comme une pratique culturelle sinon dominée, au moins marginalisée par rapport aux catégories institutionnelles de la culture légitime. On repèrera dans les catégories énoncées cette référence prédominante à ces deux critères : la " taxonomie " des faibles lecteurs relève plutôt du contenu informatif du texte que de sa structure stylistique. Et si faiblesse il y a dans la lecture, c'est probablement dans l'extrême sélectivité du contenu informatif: les thèmes et contenus énoncés se regroupent autour de la sémantique de l'«action", du "pratique ", du "vécu», selon les termes employés par nos interlocuteurs. La faible lecture manifeste sa sélectivité qualitative jusque dans le contenu du texte préféré et lu : on y retrouve l'exigence - déjà repérée au précédent chapitre - de la fonction expérimentale du texte, auquel on demande d'être en prise directe sur la vie. Le "goût » qui soutient la faible lecture se porte sur des textes évoquant la vie en action et en mouvement que signifie bien l'opposition discursive établie par cet interviewé (E 33, ouvrier pâtissier de 22 ans) entre la forme passive de l'acte de lire et l'exigence de textes évoquant le mouvement physique :

«La lecture pour moi, c'est une activité passive, dire tu es là, tu es assis, tu lis ton bouquin, tu ne bouges pas quoi, j'ai l'impression que ça ne m'apporte rien (...) Cousteau, Géo, Science et Vie, je lis, ça va là (...) comme roman rien... je lis sur le sport, le football oui (...) tout ce qui avait rapport avec l'activité que j'avais. »

\section{Le roman : une « histoire vraie, vécue »}

Les discours de nos interlocuteurs offrent un apparent paradoxe. Les uns rejettent le roman parce qu'il n'est pas suffisamment réaliste, parce qu'il relève de la fiction, les autres réclament qu'il mette en scène des expériences humaines dans lesquelles il est possible de reconnaître les siennes propres.

«J'aime bien les bouquins qui parlent d'une vie, de la vie d'un homme, d'un truc qu'il a vécu (...) quelqu'un qui perd sa femme ou quelqu'un qui divorce... des histoires vraies, vécues, un récit quoi, ça, j’aime bien le lire. »

(E 1, ouvrière, 19 ans, titulaire d'un $\mathrm{CAP}^{7}$ ).

«Les livres qui pour moi sont intéressants, c'est les livres qui apportent quelque chose au point de vu philosophique et au point de vue vie... enfin c'est des romans ou des livres de philosophie hein... les livres que j'ai lus... enfin de Frison Roche La grande crevasse, La montagne aux écritures, tous les livres de Frison Roche m'ont énormément marqué. »

Question:

«Mais ça a un rapport avec le sport aussi?»

Réponse : 
« Bien, la découverte de la montagne, l'alpinisme et la vie des gens de la montagne.

Comme Marcel Pagnol aussi (...) C'est au niveau de la vie des Provençaux-types. »

(E 29, enseignant, 38 ans). préféré des faibles lecteurs ${ }^{8}$, la faible lecture procède à une sélection thématique dans le contenu: c'est un roman qui fait office de "philosophie de la vie» - d'où cette confusion dans le discours de notre interviewé entre le genre romanesque et le genre philosophique. La faible lecture «tient le livre comme un guide de vie, comme un texte auquel on demande l'art de vivre ${ }^{9} "$.

31 L'opposition des goûts et des aversions des lecteurs pour le roman est un mécanisme qui en réalité sélectionne dans le genre les romans qui ont trait aux expériences vécues et réalistes. Le surréalisme qui exige un surplus de facultés imaginaires n'est pas dans le goût des faibles lecteurs.

Cette sélectivité dans le genre romanesque a pour effet de marginaliser certaines catégories de romans et d'en exemplariser d'autres.

\section{La science fiction : trop de fiction}

Les romans de science-fiction sont les grandes victimes de l'exigence du vécu dans la faible lecture. On leur reproche d'être trop extérieurs à l'expérience vécue :

«Je ne lis pas du tout de science-fiction, ça ne m'intéresse pas (...) tout imaginer, je ne trouve pas ça très savant. "

(E 36, représentant multicartes, 37 ans).

«Le premier truc, c'est le titre. Si c'est un titre... je ne sais pas... Les étoiles dans la nuit, non je ne l'achète pas, c'est sûr (...). Non, surtout si c'est un livre de sciencefiction. Je n'ai jamais aimé un livre de science-fiction. » (E 1, ouvrière, 19 ans).

\section{Aventures et réalisations personnelles}

Autre effet de la survalorisation du texte mettant en scène l'action et la vie en mouvement, en expérimentation, la faible lecture place très haut dans le classement des ouvrages lus, le roman d'aventures, surtout lorsqu'il évoque des réalisations physiques et personnelles. On retrouve d'ailleurs cette valorisation dans des scénarios de lecture moyenne mais qualitativement sélectifs, comme celui de cet artisan tailleur de 54 ans qui lit « beaucoup » selon ses dires, accumule des quantités énormes de livres dans son domicile, est un habitué de la bibliothèque de son quartier, mais n'a aucun diplôme parce qu'ayant interrompu sa formation scolaire pendant la seconde guerre mondiale, soit environ à la fin du cycle primaire.

"Je ne suis pas amateur des académiciens (...) j’aime bien ce qui est vécu, les histoires vécues, pas des romans..., les histoires vraies (...). Je n'ouvrirai jamais un livre de Françoise Sagan... J'aime les romans d'aventure vécue (...) un type qui fait fortune, il brasse des milliards et on ne sait plus où il en est... je voulais savoir jusqu'où il veut arriver, avec son argent et ses capitaux et j'ai été jusqu'au bout, j'ai été captivé. » (E 41)

La sélectivité de la faible lecture dans le genre romanesque, et qui exemplarise les romans relatifs aux réalisations personnelles et aux expériences vécues serait-elle commandée par les faibles niveaux d'instruction qui induisent un manque d'entraînement du lecteur à l'abstraction? La marginalisation du texte de 
mathématique dans les modélisations du livre par le genre pourrait être l'un des effets de ce mécanisme ${ }^{10}$.

\section{Le roman setntimental : des goûts non certifiés} expérimentale du livre, le roman sentimental occupe une place majeure dans la faible lecture : aux côtés des réalisations physiques et matérielles, les expériences du cœur voire les expériences érotiques - tendent à manifester le plus nettement ce modèle structural inscrit dans la pratique de faible lecture. Les interviewés le formule dans les termes de l'opposition entre le goût pour le concret propre aux gens pauvres en diplômes et le goût de l'abstrait et les choses «intellectuelles " spécifiques des gens cultivés et qui ont fait des études poussées. De fait, on retrouve cette attraction pour les genres mineurs et notamment pour le roman sentimental, chez des lecteurs moyens ou chez de grandes lectrices non diplômées ou dotées de diplômes mineurs (CAP ou BEPC tout au plus). Les classifications scolaires excluent ou dévalorisent totalement le roman sentimental ; l'une de leurs plus parfaites manifestations est le discours méprisant tenu par cette horticultrice de 27 ans, faible lectrice ayant fait des études universitaires et qui a réduit sa lecture en même temps qu'elle se déqualifiait et changeait de milieu social pour se retrouver dans un groupe d'amis de niveau d'instruction inférieur au sien :

«J'ai toujours eu un profond mépris pour toute la littérature bon marché, qu'on destine au grand public, considéré comme ignare quoi, du style de la série Harlequin, et même les polars SAS, pour moi, c'est de la basse littérature aussi. »

(E 27)

faibles lecteurs dotés de faibles niveaux d'instruction et qui lisent assidûment ces romans sentimentaux reproduisent ce discours dévalorisant sur leurs lectures préférées, et de cette façon se classifient eux-mêmes hors du registre de la lecture légitime. La faible lecture est perçue par ses acteurs comme illégitime.

\footnotetext{
«Le fait est... un roman de quatre sous n'intéresse personne. »

Question:

«Alors ce sont des romans de quatre sous que vous lisez?»

Réponse :

«Bien sûr, c'est pas des romans extraordinaires, c'est pas des romans de grands auteurs (...). Des fois, ça m'arrive, j'ai des bouquins Harlequin, des petits ${ }^{11}$ romans d'amour à l'eau de rose, bon j'en lis beaucoup, mais je n'ai pas l'impression que ça m'a fait faire un boum intellectuellement (...). C'est des petites histoires vraiment banales, toujours pareilles, mais elles sont bien écrites. Mais c'est pas ça qui m'apporte vraiment. Quand je lis un livre d'un grand auteur ${ }^{12} \mathrm{j}^{\prime}$ ai l'impression que ça m'a apporté plus. »

(E 30, femme au foyer, 64 ans, épouse d'un artisan menuisier).
}

L'enquête qualitative sur la faible lecture met en relief ce que d'autres formes d'exploration de la lecture avaient déjà relevé : la prédominance féminine dans la lecture sélective - voire exclusive - des romans sentimentaux ${ }^{13}$. Ce mécanisme ne distingue pas entre les femmes actives ou celles qui sont au foyer, pas plus que l'on y observe un effet de génération. En revanche, on voit bien apparaître l'impact du niveau de scolarisation des lectrices: c'est surtout dans les faibles niveaux d'instruction que recrute la lecture exclusive ou sélective des romans sentimentaux. De même que l'élévation du niveau d'instruction uniformise les pratiques de lecture sur le plan sexuel, il se creuse un fossé entre les sexes dans les bas niveaux d'instruction : le roman 
sentimental sont aux femmes peu instruites des classes populaires ce que les textes sur le sport est aux hommes faiblement diplômés des mêmes classes sociales ${ }^{14}$.

\section{Le livre d'histoire : de l'histoire particulière vécue à l'histoire universelle}

Si les faibles lecteurs font partie des meilleurs lecteurs d'histoire ${ }^{15}$, il convient de creuser le rapport spécifique qu'ils entretiennent avec ce genre dans lequel ils procèdent aussi à une sélection: on y retrouvera la dominante expérimentale qui commande les goûts pour certains types de romans. C'est sur l'histoire contemporaine et surtout celle de la seconde moitié du $\mathrm{xx}^{\mathrm{e}}$ siècle, relative à la période de la guerre, que la faible lecture porte un intérêt particulier: seconde guerre mondiale, guerres coloniales etc. C'est pour l'avoir vécue que les lecteurs retiennent cette histoire, comme s'ils entendaient en cela faire entrer leur histoire personnelle dans l'histoire universelle, par le passage légitimant de la littérature :

«J'aime lire des livres sur la guerre (...) parce que je l'ai beaucoup vécue cette guerre-là, de 40-45. »

(E 38, retraitée, 72 ans, titulaire d'un CAP).

Nous avons rarement rencontré dans nos scénarios des textes de romans historiques mettant en scène des périodes plus anciennes, telles que le Moyen-Age; ils s'infiltrent dans la catégorie du roman, souvent désignée par la terminologie de 1 '« histoire » :

Question :

«Pour toi donc, les livres sont des romans?»

Réponse :

«C'est des histoires. »

41 Ainsi, le roman historique semble procéder d'une logique opposée à celle qui inscrit le livre sur l'histoire dans la faible lecture : il s'agit de faire passer l'Histoire universelle dans l'histoire particulière - ou dans les histoires particulières -, le parcours conduit ici de l'Histoire universelle à l'histoire plurielle.

\section{Le livre-mode d'emploi : ouvrages pratiques sur la médecine ou sur le sport}

Le sens du « vécu » et de l'expérience qui commande la faible lecture dans la sélection "générique " des textes privilégie trois types de contenus informatifs liés à peu près tous à l'expérience quotidienne, ludique ou associative. Les ouvrages pratiques en sont un bon exemple qui guident le lecture dans son expérience pratique. Il peut s'agir d'ouvrages sur le bricolage ou le jardinage, mais aussi de textes construits en forme de guides: ouvrages de vulgarisation du droit ou de la médecine. Ici deux spécificités émergent: la forme de la lecture et le lien qu'elle établit avec la vie familiale et domestique. Ce sont généralement des ouvrages qui, comme les dictionnaires et les encyclopédies sont acquis à l'occasion d'un rite de cycle de vie ou d'une transition dans l'expérience domestique (changement de mobilier) ou dans les interactions familiales :

«Là j'ai un livre de droit que mon fils m'a prêté, sur tous les droits qu'on a, ça m'intéresse parce qu'il y a des choses qu'on connaît pas et puis avec mon fils on parlait du divorce, il veut savoir où il en est de son divorce, (...) donc il m'a dit que justement sur le livre ils expliquent bien (...). J'avais acheté des livres sur le cancer, 
parce que ma fille a eu le cancer, alors j'ai beaucoup lu sur le cancer. »

(E 38, retraitée, 72 ans). informatif renvoie à une information diffusée par les moyens de communication de masse. Ainsi on trouvera parmi ces ouvrages, des livres ou des textes essentiellement relatifs à des personnages publics et à leurs réalisations. C'est le cas de l'ouvrage écrit par Rika Zaraï, à prétention paramédicale : il a l'avantage d'associer la forme de la communication médiatique à un sujet qui s'affirme comme prophétie médicale: le savoir périphérique au savoir savant, diffusé par des moyens d'information quasi exclusifs chez les faiblement diplômés s'assimile au savoir légitime. Le livre d'actualité donne l'illusion de légitimité littéraire à des contenus informatifs diffusés par des moyens audiovisuels : il s'agit de transformer la télé en livre. scénarios de faible lecture. Lecture plus spécifique des femmes peu diplômées et non actives, elle consiste en la transformation d'une expérience individuelle exemplaire en trame romanesque: nous sommes encore dans le registre du goût pour les univers romanesques relevant des expériences "vraies et vécues". Mais là, parce que ces ouvrages sont eux aussi liés à la forme de la communication médiatique hyperpublicisée, ils ont l'avantage de familiariser des procédures expérimentales distanciées par la médiation audiovisuelle.

\section{La nature, animaux et végétaux : la « socioscience » des faibles lecteurs}

Par l'usage du terme "science » ou "scientifique », le discours des lecteurs entend légitimer des genres à thèmes très spécifiques en les englobant dans une terminologie 
générique incarnant l'institutionnalisation du savoir. En réalité, les ouvrages ou les textes scientifiques qui émergent dans la faible lecture manifestent une certaine prédominance des thèmes biologiques - animaux surtout - ou astrophysique étoiles, astres, phénomènes planétaires. La "science » définie par les faibles lecteurs ainsi réduite à certains domaines, constitue leur «socioscience» ou science différenciée par l'appartenance sociale, comme 1'«ethnoscience» est la science différenciée par l'appartenance ethnique. On observera aussi la prédominance du support-presse pour ces lectures : il s'agit le plus souvent de magazines de graphisme et de composition sophistiqués et faisant une place importante à l'iconographie, Science et Vie en particulier. La forme éditoriale de ces magazines et leur esthétique leur attribuent une valeur et un support matériels qui les assimilent à la catégorie du «livre ». Les encyclopédies fournissent elles aussi un support privilégié d'accès aux thèmes scientifiques mais la forme consultative de leur lecture les marginalise dans l'emploi du temps de la faible lecture. Le thème scientifique parait indissociable du support iconographique. Cette association se manifeste bien dans l'ordre discursif de cet ouvrier pâtissier, affirmant aimer la nature, et qui pratique l'analogie entre le «merveilleux » de la nature et celui de la communication audiovisuelle :

«Un truc sur les animaux, ça m'enrichit... comprendre leur comportement, leur vie familiale... Je trouve ça merveilleux. Je trouve la nature merveilleuse, cruelle mais merveilleuse... C'est comme un film, dès que tu vois un film et que tu sors de la salle, tu y penses encore. "

(E 33).

\section{Les marginaux : poésie, politique, sciences humaines et philosophie}

Certains genres cités et manifestement rejetés par nos faibles lecteurs ont pour logique commune d'être ceux légitimés par les cursus scolaires. De fait, ils sont clairement exclus par les lecteurs à cause du lien qu'ils entretiennent avec la catégorie « intellectuelle » ou « intelligente ». Poésie, philosophie, sciences humaines constituent ces genres "intelligents" dont l'exclusion est partagée par les moyens lecteurs sélectifs ou exclusifs - peu diplômés. Cette sélectivité renforce l'impact du niveau d'instruction dans la sélectivité "générique » de la faible lecture. En revanche, ces genres « cultivés » marginalisés, sont mentionnés mais peu fréquentés par des lecteurs en scénario de lecture décroissante - certains diplômés - et qui ont abandonné ces genres de même que les cadres sociaux de la scolarisation, milieux éducatifs et universitaires.

Ainsi les genres constituent bien des classificateurs sociaux et il est rare de constater le passage d'un genre légitime à un autre mineur, à moins d'observer en même temps des modifications dans le capital culturel du lecteur dues à une élévation de son niveau scolaire. Le passage de genres "cultivés " à des genres mineurs correspond nécessairement au passage d'un statut social et culturel à un autre. Les faibles lecteurs le savent bien qui, en même temps qu'ils affirment leur préférence pour des genres mineurs et définis par eux comme tels, concluent que ce ne sont pas leurs genres préférés qui les "enrichissent", leur "rapportent», qui leur fournissent un profit social et culturel: une manière supplémentaire de définir la faible lecture par son « illégitimité ». 


\section{Le déchiffrement du texte dans la faible lecture} l'association logique établie entre la faiblesse quantitative de la lecture et celle du déchiffrement psycho-technique ou du décodage du texte. Nos interlocuteurs nous orientaient fréquemment sur cette voie en admettant qu'ils ont du mal à comprendre certains textes, que les images sont des précieux auxilliaires du décodage de l'écrit, que la lecture représente un effort ardu de concentration, ou bien encore qu'ils ne retiennent rien ni du titre, ni du nom de l'auteur, ni même de la trame romanesque ou du contenu de l'ouvrage, tel cet ouvrier de 21 ans dont le propos est balisé par l'affirmation du manque de mémorisation et qui, avant de quitter l'enquêteur, lui avoue lire 15 à 20 livres par an, dont il a fait mention ici et là dans ses évocations. Mais d'emblée, il s'était présenté ainsi :

«Je n'ai aucune mémoire dans ce que je lis et bon je bouquine, je flirte, je bouquine une page, je lis un petit bout.»

(E 28).

L'approche psycho-technique de la lecture, perspective clinique d'une pratique culturelle dont les enjeux sociaux se manifestent à chaque étape de la démarche, depuis le choix du livre ou du texte juqu'à l'acte intime de la lecture retranchée, a tendance à négliger le poids des implications et des interactions sociales du lecteur au moment même où il parcourt le texte. Une telle approche, souvent focalisée sur les déficiences de l'apprentissage scolaire de la lecture, peut aussi négliger la dimension historique du rapport au texte, et qui se tisse dans la période de l'après-apprentissage de la lecture : ainsi cet apprentissage se poursuit-il après les premiers mois de l'école primaire et hors de l'école, et le lecteur auquel se posent des problèmes de déchiffrement de l'écrit n'est pas forcément celui qui a mal appris à lire à l'école.

Comment en effet expliquer que ceux-là mêmes qui affirment avoir des difficultés de compréhension du texte, peuvent "dévorer" certains livres, et en restituer la substance? La faible lecture comporte ainsi différents scénarios de durée et de difficulté de lecture : quelle est donc la logique de la variabilité de la lecture, entre celle qui conduit le lecteur à lire un ouvrage en quelques heures et celle qui en laisse traîner un autre pendant des mois pour finalement l'abandonner avant la fin? Aucune loi de déchiffrement du texte ne régit la faible lecture uniformément. Ce n'est pas parce qu'on lit peu qu'on lit mal. Il faut donc chercher ces variabilités du déchiffrement ailleurs que dans les compétences psycho-techniques des lecteurs. Ils nous guideront dans cette voie en affirmant qu'à chaque genre de textes correspond une forme de lecture. On a déjà évoqué les propos de cette coiffeuse de 58 ans (E 17) selon laquelle certains textes « difficiles » exigeaient plus d'efforts et de temps de lecture que d'autres. C'est presque une évidence. Cependant elle met en relief le fait qu'à l'approche d'un texte, le lecteur a déjà estimé ses propres compétences de déchiffrement en fonction de son décodage de la nature du texte, et de sa classification dans la hiérarchie des genres. Ainsi pourraiton dire que le lecteur a les compétences de déchiffrement pour le texte qu'il classe au niveau de ses compétences. Parlant de ses livres scolaires, un jeune ouvrier affirme ainsi :

«Je ne les ai pas lus dans le sens où l'on peut entendre lire, essayer d'approfondir, pas suivre sur des lignes et oublier quelque temps après, je n'ai pas suivi, j'ai lu ce qu'il y avait d'écrit, peut-être que je n'ai pas assez eu de connaissances de la part... c'est-à-dire qu'on ne m’a pas proposé des livres qui étaient dans mon créneau, c'est 
tout $^{16}$ »

(E 28, ouvrier 21 ans, titulaire d'un brevet d'études professionnelles BEP).

faible lecture semble focaliser sa faiblesse dans la difficulté d'appréhender l'écrit; quelques uns de nos interlocuteurs situés dans les tranches d'âge 55-70 ans et plus, et ceux en particulier de faibles niveaux d'instruction (non diplômés ou titulaires de CAP ou de (EP) affirmaient être rebutés par la finesse et la petitesse de certains caractères d'imprimerie et ne choisir que les livres imprimés en gros caractères. D'autres, plus jeunes et parmi ceux en particulier qui sont issus du système scolaire juste après l'âge minimal de scolarisation, rationalisaient leurs difficultés de lecture par l'aversion pour l'écrit, que cet interviewé définit comme une « ligne continue » :

«Alors Gaston Lagaffe, ça oui, parce qu'il y a des images, c'est vrai, parce qu'il y a de l'écrit et de l'image, alors quand il n'y a que des lignes, je n'ai pas envie d'imaginer... je n'ai pas envie d'entrer... je pourrais mais ça serait vraiment un effort, ce n'est pas un plaisir de lire, c'est un effort. »

(E 33).

Et plus loin, la relation entre lecture et écriture :

"C'est parce que je n'aime pas lire que je n'aime pas écrire. De toute façon, comme je n'ai jamais lu, le style n'est pas venu. Comme je n'étais pas doué et que je ne lisais pas, je n'étais pas bon en dissertation. "

(E 33, ouvrier pâtissier, 22 ans).

58 Ainsi c'est parmi les moins bien diplômés, les moins bien entraînés au décodage linguistique que l'on repère des scénarios de faible lecture constitués sur des difficultés de déchirement. C'est probablement la raison pour laquelle ces scénarios incluent la lecture assez soutenue de la presse hebdomadaire mensuelle ou quotidienne où le texte écrit, bien qu'imprimé en caractères fins et de petite dimension, est structuré, décomposé et pré-mâché ou pré-décodé par la présence de titres et de sous-titres et par la différenciation des caractères qui suggère une classification des informations.

Ce qui ressort distinctement des mécanismes de déchiffrement des textes dans la faible lecture c'est l'impact conjoint du niveau d'instruction et des réseaux de socialisation des lecteurs. Le déchiffrement est peu favorisé par des trajectoires scolaires articulées dans des processus d'échec; il ne peut également être soutenu que par les cadres habituels d'interaction et de communication. Les modalités de cette conjonction sont 
variables mais d'une manière générale, ces réseaux ne soutiennent, pour la faible lecture que le déchiffrement de certains types de textes. Ce qui ressort clairement de cet ensemble de modalités qualitatives, c'est que d'une part, il est sociologiquement impossible de démontrer qu'à la faiblesse quantitative de la lecture de livres correspond la faiblesse qualitative du déchiffrement ${ }^{17}$ et que d'autre part, les faibles lecteurs ou la faible lecture sont loin de recouvrir les univers sociaux de l'illettrisme.

\section{NOTES}

1. Ouvrier pâtissier. 22 ans, célibataire vivant dans une petite commune d'Alsace (E 33).

2. Pour des raisons sensiblement proches, le livre ne peut constituer une valeur d'échange, être intégré dans une transaction de type potlatch que constitue le cadeau; la faible lecture, parce qu'elle est illégitime ne peut accorder au livre une valeur d'échange ; le livre ne peut soutenir des interactions qui ne le supportent pas. Le discours manifeste cette exclusion, lorsqu'il l'explique en termes de difficultés d'ajuster le livre offert au "goût» de la personne. Les lecteurs plus familiers avec le livre ne se poseront pas la question du goût du destinataire si ce dernier fait, comme eux, partie des gens qui ont le "goût de la lecture ». Chez les faibles lecteurs en revanche, le doute qui pèse sur la présence du livre dans les "goûts " personnels manifeste la remise en cause de sa valeur d'échange : plus que personnels, ce sont des goûts sociaux qui sont désignés :

"Quelquefois, il y en a qui ne pensent pas à nous offrir des livres, parce qu'il faut tomber dans les gôuts (...) il y a des livres qui vous sont chers et ils ne sont pas tellement appréciés. Il faut que ça provoque une curiosité».

(E 18, coiffeur, 59 ans, résidant à Caen, titulaire d'un CEP).

3. MAUSS Marcel. DURKHEIM Émile. De quelques formes primitives de classification. MAUSs Marcel, Essais de sociologie. - Paris : Seuil, 1969. pp. 162-230.

4. PARMENTIER Patrick, Les genres, le classement des lecteurs et le classement des livres Trois études sur la lecture, op. cit., pp. 11 et suivantes.

5. Pratiques culturelles des Français, op. cit., p. 68.

6. Voici la liste des catégories énoncées dans les Pratiques culturelles: dictionnaires et encyclopédies; romans autres que policiers ou d'espionnage; ouvrages pratiques; livres sur l'histoire; romans policiers ou d'espionnage; œuvres de la littérature classique; livres pour enfants; bandes dessinées; livres d'art; beaux livres de photographies; livres scientifiques, techniques, professionnels; livres de poésie; essais politiques, philosophiques, religieux, de psychologie ; reportages d'actualité.

7. C'est nous qui soulignons.

8. Cf. Brefs repères sur les faibles lecteurs... op. cit.

9. BOURDIEU Pierre. La lecture, une pratique culturelle. Pratiques de la lecture, op. cit. p. 227.

10. Voir p. 83.

11. C'est nous qui soulignons.

12. Dans la catégorie des « grands auteurs » on retrouvera essentiellement les grandes figures de la littérature romantique, naturaliste ou populiste du XIX siècle : Hugo, Balzac, Zola, Stendhal.

13. Cf. PARMENTIER Patrick, op. cit.

14. Les mêmes mécanismes produisent des effets spécifiques de génération : la bande dessinée est aux jeunes peu diplômés ce que les ouvrages sur la médecine sont aux retraités ou aux adultes 
s'approchant de la retraite. Creusant des distinctions hiérarchisantes dans les genres, les niveaux d'instruction creusent aussi les distinctions d'âge. Le cas de la bande dessinée est quelque peu ambigu cependant dans la mesure où elle est aujourd'hui reconnue comme genre littéraire voire artistique - légitime et inclus dans les goûts "savants» des intellectuels. Elle reste néanmoins un genre spécifique des pratiques des jeunes peu diplômés des classes populaires, même si à l'intérieur du genre s'opère une sous-classification qui «illégitime " les Lucky Luke, Astérix, Super Héros apparentés plutôt au style du roman-photo qu'à celui du dessin artistique mis en scène.

15. Données complémentaires sur les faibles lecteurs.... op. cit.

16. C'est nous qui soulignons.

17. Les méthodes d'observation manquent d'adéquation: comment faire émerger ces mécanismes en situation clinique - le lecteur interrogé pendant la lecture ou tout de suite après - ou en situation parfaitement distanciée - et enregistrer l'ampleur de la mémorisation, sans introduire à chaque fois le déchiffrement du texte dans des conditions limitées au plan sociologique? 


\section{Troisième partie. Le livre et la lecture : perception, modélisation, représentation}

1 L'espace des représentations du livre et de la lecture chez les faibles lecteurs, se présente comme le terrain de l'équivoque et du paradoxe. En réponse à la question générique de l'enquêteur sur «ce que représentent le livre et la lecture » et sur les modélisations de la pratique et de l'objet seront avancés les schémas légitimés par la culture dominante avec le poids de l'auto-dévalorisation d'un lecteur qui se place d'emblée à l'extérieur de ces schèmes. D'une certaine manière, ces représentations de la lecture légitime consistent pour le lecteur à mésestimer la sienne propre et à s'identifier comme faible lecteur :

«Ce n'est pas moi qui les mets en haut de l'échelle (les bons écrivains), c'est les autres, moi je ne m'y connais pas assez (...), il y a des gens plus lettrés que moi pour les classer, moi je ne suis qu'une petite roue ${ }^{1} . .$. »

En contrepoids, les représentations spécifiques qui émergent des pratiques, tendent à formuler un modèle de lecture différenciée qui, tant dans la sélection et la classification des genres, dans l'acquisition des livres que dans le rapport au contenu de l'ouvrage, s'opposent aux modèles légitimes avancés par nos interlocuteurs dans leur discours général sur la lecture. En fait, la faible lecture apparait comme une pratique dominée par des représentations génériques conformes aux modèles légitimes. La spécificité de la faible lecture est à situer dans ce fossé creusé entre lecture légitime et lecture différenciée dans la modélisation dominante, aussi bien lorsqu'elle est formulée dans le discours institutionnel que lorsqu'elle est incorporée par ceux qu'elle marginalise.

\section{Le lecteur, un « professionnel » de la lecture ${ }^{2}$}

Cette dichotomie classifiante du discours sur la lecture se manifeste déjà, au plan des représentations générales, dans la manière d'identifier le lecteur sur le mode de l'archétype: il est un intellectuel, cultivé, pratiquant un métier intellectuel, un 
«professionnel» de la lecture qui systématise sa consultation des ouvrages et qui dispose d'éléments psycho-techniques et sémantiques de déchiffrement des textes :

4 Certes le discours de l'horticultrice de 27 ans, ex-étudiante en lettres est à ramener à sa volonté de se distinguer de son milieu immédiat de faibles lecteurs en rappelant les modèles conformes à son origine sociale. Elle nous dira par exemple des lecteurs qui lisent beaucoup, qu'ils "font partie de la classe des «intellectuels», du corps professoral littéraire, les professeurs de philo, de français, d'histoire ». Mais à côté de ces attributs professionnels, les lecteurs disposent d'attributs personnels : ils sont des "passionnés de lecture", ils ont le goût pour la lecture, nous diront d'autres interlocuteurs, comme cet enseignant qui affirme que les lecteurs assidus sont ceux qui " sont penchés sur la lecture, qui ont un certain mode de vie qui est axé là-dessus » (E 29).

5 La lecture légitime est donc plus qu'une pratique isolée, elle constitue un «mode de vie ». La distinctivité de la formule « lecteur » s'accompagne de la nécessité de posséder des " goûts » distinctifs. C'est pourquoi cet archétype de lecteur se trouve à l'opposé du faible lecteur qui se définit lui-même comme tel: il n'est pas intellectuel, il ne lit qu'accessoirement et la lecture n'est pas dans ses " goûts ». Ou bien il ne disposera que de l'un de ses deux attributs complémentaires : s'il a le goût de lire, il n'en a pas les moyens sociaux; s'il en a les moyens sociaux, la lecture fait partie de ses choix secondaires.

\section{La lecture : apprendre, se détendre, s'évader, définition fonctionnelle}

6 Nous laisserons de côté la représentation rebattue de la lecture comme acte de culture par excellence et d'élévation sociale ${ }^{3}$, nous nous attarderons plutôt sur les fonctions ludiques attribuées à la lecture qui nous paraissent relever de la spécificité de la faible lecture, comme paraissait le souligner l'étude de Nicole Robine sur les jeunes travailleurs et la lecture ${ }^{4}$. La terminologie de la lecture-loisir est pourtant encombrante; lorsqu'elle est d'emblée formulée dans l'interrogation de l'enquêteur, elle a tendance à extraire la lecture du cadre de la socialisation professionnelle, associative ou familiale pour la transformer en retraite intime et tout à fait individuelle.

7 La lecture envisagée comme une « détente », une " évasion », un " dépaysement ${ }^{5}$ », un encouragement au rêve, par ceux-là mêmes qui affirment préférer les textes qui traitent du "vécu», «de choses et de faits réels » ayant trait à la vie pratique et concrète, se positionne, dans les représentations hors du champ social de l'expérience. Cet archétype de lecture, parce qu'il est perçu comme lecture légitime, est placé par le discours de nos interlocuteurs à l'extérieur de leur expérience sociale : se détendre, se dépayser, s'évader en lisant permet d'affirmer cet effet de fuite et surtout de distance que l'on perçoit dans la pratique lectorale. La lecture est en quelque sorte le "négatif " du vécu ordinaire. C'est la raison pour laquelle le temps qui lui est attribué ne peut être que celui de l'anti-travail (à opposer au temps libre du loisir), un temps résiduel. Cette forme de temps envisagé pour la lecture est le principe interprétatif du fameux leitmotiv «je n'ai pas le temps de lire», traduire «je n'ai plus le temps résiduel à consacrer à la lecture, parce que la lecture pour moi ne peut être que dans ce temps du 
non actif, du négatif, de l'opposé à la vie réelle, et jamais programmée comme pratique courante et légitime de mon mode de vie ».

C'est dans la même logique que le seul mode de lecture envisagé est celui qui consiste à lire un ouvrage de bout en bout, en lecture pleine et qui exige que l'on consacre une portion programmée de temps. La lecture partielle ou consultative d'ouvrages tels que les encyclopédies ou les dictionnaires, n'est pas rangée dans la catégorie archétypique $\mathrm{du}$ « Lire ».

9 Enfin c'est encore dans cet ordre d'idées génériques que sont exclues les lectures à usage ciblé et professionnel, en particulier, de la catégorie légitime de la «Lecture ». Celle-ci ne peut-être qu'une détente, pour ceux qui ont le « goût » ou la passion pour la lecture, mais ce n'est pas lire que consulter des ouvrages techniques, juridiques, etc. dans un but professionnel.

10 Ainsi, cet enseignant alsacien nous répond-il à la question du nombre de livres lus en n'y incluant que les ouvrages lus pendant la détente, et non ceux consacrés à la préparation de ses cours (E 2).

11 De même cet ouvrier qualifié d'EDF, interviewé avec son épouse, une lectrice assidue de romans sentimentaux, qui range ses brochures professionnelles dans un placard, ne les considérant pas comme objets de lecture, comme objets culturels, et par conséquent, se définissant comme "non lecteur" du fait que ces textes professionnels sont ses uniques lectures avec celle de la presse quotidienne (E 12).

12 Enfin, un technicien des Postes et Télécommunications, non diplômé, nous explique qu'il a «décollé de la lecture» parce qu'il ne lit plus que des textes à usage professionnel : la lecture est pour lui :

«Une façon comme une autre de sortir du milieu habituel (...) ça apporte des informations extérieures..."

13 En revanche, au sujet des livres à usage professionnel, notre technicien affirme qu'il pratique

« une lecture plus... qui a un rapport avec mon travail, directement ou indirectement, qui m'apporte des informations techniques au niveau de mon travail. "

(E 16, technicien PTT, 30 ans, non diplômé).

14 Mais le plus paradoxal dans cette définition restrictive de la lecture légitime par ceux qui n'y ont pas accès, c'est qu'elle exclut partiellement la lecture de la presse quotidienne en particulier - pratique ponctuelle d'acquisition d'information de " petit gabarit » et qui ne fait pas l'objet d'un effort d'accumulation du savoir. Car c'est bien en cela que la « lecture » consiste : lire c'est accumuler des connaissances, c'est capitaliser.

\section{Le livre : définitions matérielle, graphique, par le genre ou par l'auteur}

15 Il est toujours équivoque d'enquêter par la terminologie du livre, car le poids des mythes s'impose sur l'usage des mots.

16 La terminologie du livre est aujourd'hui empruntée pour désigner des univers sociaux de structures très diverses mais regroupés sous la catégorie générique et sémantique universelle du livre : on dira "les ouvriers du livre» - en y incluant aussi ceux de la presse -, le salon du livre et non des livres, etc. Le livre est en quelque sorte une 
abstraction valorisante qui lui permet d'une part d'être assimilé à la culture par excellence $^{6}$ et d'établir la distance nécessaire qu'impose tout culte. C'est pourquoi l'usage de terme "bouquin" familiarise, démythifie et d'une manière générale caractérise des lecteurs proches du livre, aux plans matériel et intellectuel. Ou bien, et plus généralement, le terme "bouquin", désignera des ouvrages quelque peu dévalorisés, soit par leur forme éditoriale - poche, petit format - soit par leur contenu - collection Harlequin et ouvrages à thèmes non légitimes.

\begin{tabular}{|c|c|c|c|c|}
\hline \multicolumn{1}{|c|}{} & Définition & Fonctionelle & Formelle \\
\cline { 2 - 5 } & Lecture & Loisir-détente & Pleine \\
\hline Anti-lecture & Professionelle & Consultative \\
\hline \multirow{2}{*}{ Définition } & Matérielle & Genre & Auteur & $\begin{array}{c}\text { Rapport } \\
\text { texte/image }\end{array}$ \\
\hline Livre & $\begin{array}{c}\text { cartonné } \\
\text { relié }\end{array}$ & $\begin{array}{c}\text { littérature } \\
\text { romanesque }\end{array}$ & $\begin{array}{c}\text { classique ou } \\
\text { contemporain }\end{array}$ & $\begin{array}{c}\text { texte } \\
\text { prévaut }\end{array}$ \\
\cline { 2 - 5 } Anti-livre & $\begin{array}{c}\text { livre } \\
\text { multigraphié }\end{array}$ & inconnu & $\begin{array}{c}\text { image prévaut } \\
\text { B. D. }\end{array}$ \\
\hline
\end{tabular}

Tableau 2 : Les représentations du livre et de la lecture

Les représentations enregistrées chez nos interlocuteurs formulent quatre critères de définitions du livre : sa forme éditoriale, son genre, son auteur, et le rapport qui y est établi entre le texte et l'image. Ces critères apparaissent le plus souvent complémentaires et non pas exclusifs l'un de l'autre.

\section{La forme éditoriale}

La terminologie du "livre», telle qu'elle se manifeste dans les discours de nos interlocuteurs, privilégie d'emblée la définition matérielle du livre : c'est d'abord à un ouvrage "cartonné", relié et broché qu'ils font allusion, un ouvrage qu'on aime " conserver ». C'est donc dans sa fonction de "capital culturel objectivé », tel que le définit Pierre Bourdieu ${ }^{7}$, que la catégorie du livre est formulée dans un premier temps :

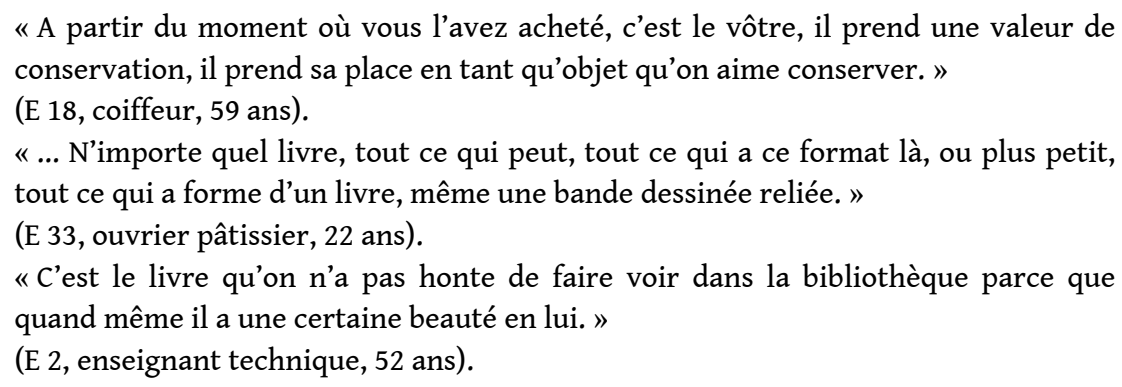

Cette définition matérielle du livre renvoie donc à des pratiques d'appropriation du livre et à la fonction ostentatoire des stocks privés d'ouvrages conservés à la maison. De ce point de vue, elle tend à rationaliser certains scénarios pratiques de lecteurs pour lesquels l'appropriation matérielle du livre apparaît tangiblement comme la tendance à s'identifier, au moins à ce niveau matériel, aux modèles légitimes de la lecture « cultivée ». 
20 La définition matérielle du livre apparaît restrictive lorsqu'elle marginalise les imprimés dont la forme éditoriale, format et volume, ne sont pas conformes aux normes de l'édition officielle et dont la diffusion est restreinte: imprimés à usage professionnel, limités à la diffusion interne d'institutions professionnelles, et non «exposés »: leur marginalisation dans les représentations du livre les exclut du domaine du visible; ils seront enfermés à l'intérieur d'armoires non vitrées. Et si les revues, illustrés et magazines sont exclus eux aussi de la catégorie du livre, ils pourront y être intégrés s'ils font l'objet d'une collection reliée et stockée dans les bibliothèques privées.

21 Les périodiques dont la mise en page est luxueuse - esthétique du papier, du caractère, de l'iconographie - sont ainsi en position marginale. Certains d'entre eux, régulièrement lus et présents au foyer pendant plusieurs semaines après l'achat, peuvent être désignés par le terme «livre» que semble appeler l'amoncellement anarchique mais visible des textes empilés en forme de bibliothèque de fortune.

22 C'est dire combien la matérialité du livre-objet importe dans la représentation du livre, combien la forme «objectivée » du livre détermine sa perception et les mythes qu'il produit.

\section{Le genre}

C'est le genre romanesque qui émerge des discours comme la catégorie incarnant le « livre » par excellence. Mais ce n'est pas à la définition officielle et savante du genre que les discours se réfèrent, c'est plutôt à une définition fonctionnelle.

« Une histoire (...) soit réelle, soit imaginaire, qui commence et qui a une fin. »

(E 33, ouvrier pâtissier, 22 ans).

24 Cette définition du livre par le genre littéraire n'est pas fortuite. On y trouve celle, formelle, de la lecture. Le livre désigne dans son contenu un texte synthétique et homogène dont on ne peut lire un chapitre pour en abandonner un autre. On ne peut lire le « livre » que dans un ordre particulier, du début jusqu'à la fin. Le livre est un texte plein comme la forme de lecture légitime.

Cette définition exclut donc les ouvrages dont le genre les limite à une lecture consultative et ponctuelle : encyclopédies, dictionnaires, livres de recettes, et que l'on ne comptera pas dans la quantification des livres lus : c'est à la fois le genre et la forme de lecture qu'ils supposent qui les marginalisent.

Autre particularité de la définition : le genre romanesque hyper-valorisé, marginalise les ouvrages scientifiques ou mathématiques. Le vrai livre est considéré par nos enquêtés, comme un ouvrage ouvert sur l'imaginaire, sur le rêve et aussi sur l'évocation de trames réalistes que ne suggèrent ni la construction systématique, ni l'abstraction des textes scientifiques ou mathématiques.

On retrouve dans la définition du livre par le genre, comme dans sa définition matérielle, une certaine conformité à la spécificité pratique de la faible lecture. Rappelons en effet que parmi les genres préférés des faibles lecteurs ${ }^{8}$, le roman non policier occupe une place privilégiée. Mais l'ouvrage pratique et le dictionnaire bien placés eux aussi dans les préférences des faibles lecteurs ne sont pas autant valorisés par les représentations du livre. Le roman est le genre qui légitime la pratique. Pourront également y être inclus des genres qui, distants par la forme, se rapprochent 
du roman par leur contenu. Ainsi certaines biographies d'acteurs ou de vedettes de variétés, racontées comme en forme de roman, seront assimilées à cette catégorie modèle, et ce d'autant plus qu'elles conjuguent la concrétude des faits à la formulation romanesque d'une expérience personnelle similaire à un conte de fée, exemplarisée par le genre.

\section{L'auteur}

La représentation du livre légitime désigne un ouvrage écrit par un "grand auteur ", écrivain classique ou contemporain, reconnu soit par le medium scolaire - livres de classe - soit par les medias "cultivés » - l'émission Apostrophes en particulier et d'autres émissions sur les livres. Ainsi auteurs inconnus ou hyper-connus comme Barbara Cartland qui plus qu'un auteur devient une désignation de collection, sont manifestement marginalisés dans la catégorie du livre. On a vu qu'ils sont exclus également des stocks visibles de livres conservés. Cette définition du livre par l'auteur légitime s'ajuste donc sur les formes d'appropriation matérielle du livre. Néanmoins elle s'oppose à la sélection pratique des auteurs qui privilégie justement les «illégitimes », les exclus de la culture dominante et savante :

29 Ainsi cette femme au foyer de 64 ans (E 30) épouse d'un artisan retraité, nous explique que les livres qu'elle a lus, parfois de manière intensive, ne lui ont pas "apporté »entendre « rapporté » au sens de profit culturel - parce que c'étaient des «livres de quatre sous » qui n'ont rien à voir avec ceux écrits par les grands auteurs.

De fait, en traçant un fossé entre cette représentation du livre-modèle écrit par un « grand auteur » - ou auteur légitime - et le livre qu'il préfère lire, le faible lecteur tend à formuler la non légitimité de sa pratique elle-même. La représentation de la lecture chez les faibles lecteurs les marginalise en tant que lecteurs parce qu'elle dévalorise leur pratique de lecture.

31 La mention de l'auteur comme critère de définition du livre apparaît plus souvent dans les représentations des lecteurs dont la pratique de lecture est affaiblie ou en réduction, et issus de catégories sociales mieux dotées en capital culturel que les faibles lecteurs en scénario stable, issus de classes populaires le plus souvent.

32 En principe la mention de l'auteur, surtout si elle est spontanée, s'inscrit dans la volonté de se distinguer comme détenteur du " goût " pour la lecture « cultivée » et qui se réfère aux classifications dominantes et savantes du livre par son auteur.

\section{Le rapport texte/image}

Le livre par excellence est essentiellement écrit. Il est dominé par la présence de texte et comporte peu d'images. L'iconographie y a une place secondaire.

Cette définition graphique du livre met en évidence la relation étroite qu'il entretient avec l'écriture dans les représentations, et également avec la langue. La qualité du style et de la langue utilisée par l'auteur apparaît comme l'un des critères prioritaires d'estimation des ouvrages, surtout chez les faibles lecteurs démunis de diplômes et pour lesquels le livre est un peu leur école : c'est l'apprentissage de la langue et de son usage qui sera exigé dans la lecture. Mais ici on trouvera la spécificité du discours de certaines générations de lecteurs dont la scolarisation a été freinée par la seconde 
guerre mondiale, ceux qui issus de milieux populaires, ont cessé d'aller à l'école à cette époque et n'y sont pas retournés après la guerre.

Cette définition du livre, comme texte essentiellement, permet de marginaliser radicalement la bande dessinée, non seulement parce que l'image y prédomine, mais surtout parce que la langue employée - jargons et argots ou néologismes non encore intégrés dans le dictionnaire - n'est pas considérée par nos enquêtés comme langue légitime et savante.

Ce n'est pas le cas pourtant du groupe d'adolescents interrogés dans leur lycée d'enseignement professionnel (LEP) de la banlieue parisienne et qui lisent presque exclusivement des bandes dessinées, comme certains jeunes adultes tout juste sortis du système scolaire. La lecture de la bande dessinée qui privilégie l'image, se présente pratiquement comme le rejet des normes de l'apprentissage de la langue dominante.

Le rapport entre le texte et l'image dans la définition du livre est celui qui distingue la langue légitimée par la culture dominante de celle usitée dans la culture dominée. Les faibles lecteurs manifestent bien ce fossé lorsque les uns présentent la faiblesse de leur pratique de lecture comme l'effet du manque de maîtrise de la langue légitime, et les autres l'arborent comme le refus de s'identifier socialement et culturellement à elle. Effet de génération et effet de domination culturelle se rejoignent dans ces représentations.

Un autre paradoxe produit par la survalorisation du texte dans la définition du livre est la marginalisation du livre d'art, pourtant privilégié par la définition matérielle du livre. La prédominance de l'iconographie dans ces ouvrages les transforme en musées mobiles. On ne lit pas un livre d'art, nous dit-on, on le regarde, comme on visite une exposition ou un musée. Il faut dire que la population de faibles lecteurs fréquente très rarement les expositions et les musées. On distinguera cependant entre livres d'art où l'iconographie est support de communication, où elle est texte, et les ouvrages où l'image illustre un texte. Ces derniers sont moins marginalisés parce que l'image permet le déchiffrage du texte, elle est l'auxiliaire du texte. Lorsque l'image est le texte, elle suppose des formes spécifiques de décodage que les faibles lecteurs, de par leurs caractéristiques et itinéraires sociaux, ne maîtrisent pas (par l'impact du niveau d'instruction et de la déqualification ou de la mobilité sociale descendante notamment ${ }^{9}$ ).

L'ensemble structuré de ces représentations apparait principalement antithétique des pratiques des faibles lecteurs. Elles permettent donc à nos interlocuteurs de se placer d'emblée comme des non lecteurs, la catégorie du «faible " lectorat n'étant jamais par eux évoquée: on est ou on n'est pas lecteur. Nous sommes dans le registre des définitions absolues, on comprend mieux pourquoi bon nombre d'entre eux repoussaient, dans un premier temps, notre démarche d'entretien sur le thème de la lecture : «Je ne suis pas concerné ! ».

Ces représentations divergentes des pratiques nous permettent de mieux repérer ce que l'on entend par lire "faiblement", en termes de définition qualitative; la faible lecture se présente dans cette articulation comme :

- une lecture non planifiée et non systématique ;

- une lecture consultative, ponctuelle et partielle ;

- une lecture d'auteurs non reconnus par la lecture savante ;

- une lecture de livres non capitalisables, qui circulent et que l'on ne garde pas ; 
- une lecture de genres et d'auteurs mineurs, non légitimée par la culture dominante ;

- une lecture qui semble parfois privilégier l'image sur l'écrit (magazines, presse soutenue par

l'iconographie, bandes dessinées, romans-photos, etc.

\section{NOTES}

1. Il s'agit là pourtant d'un lecteur moyen qui illégitime sa pratique par son niveau d'instruction et sa sélection en genres, similaire à celle des faibles lecteurs quantitatifs. La représentation enregistrée vise à le transformer en un faible lecteur qualitatif (E 41, artisan-tailleur, 54 ans, non diplômé).

2. A distinguer de la lecture à usage professionnel.

3. Lire peut être perçu comme un moyen de connaître " des gens plus cultivés », de vrais lecteurs, des lecteurs légitimes.

4. ROBINE Nicole Les jeunes travailleurs et la lecture. - Paris : Documentation Française. 1984. p. 131.

5. C'est le cas pour la lecture de textes consacrés à la connaissance des contrées et de sociétés exotiques, support du voyage.

6. "Le livre, c'est la culture par excellence» (E 36, agent commercial, 39 ans, titulaire d'un baccalauréat technique).

7. «Le capital culturel objectivé dans des supports matériels tels que des écrits, des peintures, des monuments, etc., est transmissible dans sa matérialité ». Cf. Les trois états du capital culturel Actes de la recherche en sciences sociales. $n^{\circ} 30,1979$, p. 3.

8. Données sur les faibles lecteurs, op. cit.

9. S'ils recouvrent la plus grande partie de l'échelle sociale, ils sont néanmoins très peu représentés dans les catégories les plus fortes consommatrices de l'art : enseignants, spécialistes de la culture institutionnelle et professions intellectuelles plus généralement. Cf. Pratiques culturelles des Français, op. cit.. p. 150 et suivantes+154 et suivantes. 


\section{Conclusion}

1 Bien que limités par les déterminations quantitatives de cette étude, qui tendent à présenter un groupe statistique comme réalité sociale homogène, nous nous étions proposés de saisir une pratique recouvrant un faisceau de trajectoires, dans la diversité de ses modalités. Il ressort de la consultation répétée des matériaux et de la récurrence de certaines modalités pratiques ou sociales, que la faible lecture paraît commandée par un modèle structural impliqué à tous les niveaux de la démarche du lecteur. Quelles sont donc ces formes et les mécanismes qui tendent à se regrouper sous ce modèle?

2 La première manifestation procède du discours et de la présentation ou de la représentation de soi du faible lecteur : il se définit comme lecteur par sa trajectoire scolaire et surtout rationalise la faiblesse de sa démarche en termes d'échec scolaire et de «domination » culturelle : le faible lecteur s'envisage d'emblée comme démuni de «capital culturel » et donc de moyens sociaux d'accéder à la lecture reconnue par lui comme légitime. De fait, c'est donc toujours en rapport avec la légitimité de la lecture savante que la faible lecture s'articule et se définit, plutôt que dans ses modalités et ses conditions intrinsèques. De ce point de vue, même si elle apparaît à l'enquêteur comme une pratique différenciée qui comporte sa logique sociale propre, elle s'affirme d'abord comme pratique illégitime. Les circonvolutions discursives des lecteurs sur la justification de la faiblesse de leur rapport au livre en sont d'évidentes formulations.

Autre manifestation «structurale » de la faible lecture, sa sélectivité et sa spécificité :

- dans les moyens de communication de l'information sur le livre et de diffusion des ouvrages : on relève la prédominance des moyens de communication de masse et de réseaux de commercialisation en grandes surfaces, qui s'opposent paradoxalement au pôle privé des relations familiales;

- dans l'emploi du temps de la lecture : le livre figure ici en grand marginal, occupe les temps morts, laissés libres par les activités majeures - on lit si on n'a rien d'autre à faire. C'est ce qui explique sans doute ce leitmotiv du refus des faibles lecteurs à y investir l'« effort» nécessaire ; le livre est ici dans le registre du passif ;

- enfin, et c'est là une des spécificités majeures de la faible lecture : elle sélectionne des "genres » thématiques où l'univers narratif est dominé par 1'«action », le "vécu», le "pratique » et qui n'ont pas reçu la légitimation institutionnelle de l'école (ouvrages ne risquant pas de se trouver dans le Lagarde et Michard, recueil de textes littéraires destinés aux élèves de l'enseignement secondaire). 
Le livre faiblement lu s'inscrit dans des réseaux d'interactions sociales et de communication où il ne figure ni en tant qu'objet culturel ni en tant qu'objet spécifique. Il semble s'infiltrer presque en clandestin dans la masse d'informations et de relations qui évoluent dans divers domaines de l'expérience sociale. La lecture de livres ne semble donc pas, pour les faibles lecteurs, constituer une forme de "capitalisation culturelle», elle n'est pas effectuée en vue d'acquérir du capital culturel. Les lecteurs le savent bien qui reconnaissent que les genres de livres et de magazines qu'ils préfèrent ne leur « rapportent " pas au plan intellectuel, pas plus que la place que tient le livre dans leur emploi du temps ne saurait leur permettre un tel profit. La lecture ne figure pas non plus parmi les loisirs préférés des faibles lecteurs ${ }^{1}$. Elle est là sérieusement concurrencée, non pas par la télévision, mais par les activités physiques et les rencontres familiales ${ }^{2}$. D'où la représentation de la lecture comme activité passive, qui n'offre pas la possibilité du mouvement. Il faut sans doute ajouter à cette remarque l'analyse du "capital culturel incorporé " impliqué dans la faible lecture. Selon Pierre Bourdieu, cet «état » du capital culturel exige un investissement personnel et physique nécessaire à « l'incorporation » du savoir. Dans la faible lecture, l'incorporation du capital culturel semble manifestement minorée par les réticences des lecteurs à investir physiquement et personnellement dans cette pratique. C'est sans doute la raison pour laquelle les événements physiques que constituent les maladies et les séjours à l'hôpital sont évoqués comme des moments exceptionnels dans la carrière lectorale : à vrai dire, ils libèrent nos interlocuteurs des activités "personnalisées et incorporées » habituelles qui ne leur laissent aucune place pour la lecture, dans le temps ordinaire.

5 Ni mouvement physique, ni mouvement social : les acquis de la lecture ne font que très rarement l'objet d'échanges ou d'interactions sociales : si la lecture est ici soutenue par les cadres sociaux familiers (télévision ou famille), elle n'est pas un stimulateur de rapports sociaux, il est rare qu'elle fasse l'objet d'échanges intellectuels ou même pratiques, sauf pour certains ouvrages consultés très ponctuellement ${ }^{3}$. S'il est un guide de vie, le livre met rarement en place des actes sociaux effectifs. Et si la télévision fait parler du livre après la diffusion d'une émission, le livre n'introduit pas les lecteurs dans de nouveaux horizons sociaux. Les lecteurs paraissent ainsi exprimer un vœu pieux plutôt qu'une réalité lorsqu'ils affirment que la lecture permet de rencontrer ou de « parler avec des gens plus cultivés » (qu'eux).

6 Ce modèle structural qui semble commander la faible lecture fait apparaître la conjonction de deux types d'effets :

- Le premier est relatif au niveau d'instruction : la faible lecture est dominée par les effets de la faiblesse du niveau scolaire ou de l'échec de la scolarisation. La faible lecture, dans les genres et les modes choisis, est une lecture non certifiée, non sanctionnée par l'institution légitimante. La forme institutionnalisée du capital culturel lui fait défaut.

- L'origine sociale et les cadres de la socialisation des lecteurs se conjuguent dans les scénarios de faible lecture pour dénier à la lecture un profit social ou culturel. Les scénarios de faible lecture évoluent dans des milieux sociaux où la lecture ne peut permettre la constitution d'un capital social, effet de la faiblesse des niveaux d'instruction qui ne permet pas l'accès à ces réseaux sociaux où l'on « capitalise » en lisant.

7 On ne peut manquer d'être frappé par une apparente contradiction entre la socialisation de l'acquisition du livre et de l'information sur le livre dans les réseaux habituels des lecteurs, et l'absence de socialisation de la lecture comme acte 
d'engagement physique. Tout se passe comme si le social disparaissait progressivement du scénario lorsque le livre entre dans l'univers intime de l'incorporation individuelle. Les lecteurs signifient cette disparition dans la contradiction formulée entre leurs pratiques effectives et leurs représentations de la lecture et du livre. Si la représentation de soi du faible lecteur exprime sa condition de domination culturelle, et si la faiblesse de sa pratique lectorale se manifeste par un processus de désocialisation ou de manque de soutien social, il n'en reste pas moins que les connaissances acquises lui permettent de maintenir sa conscience d'appartenir à la culture majoritaire, transmise par toute la gamme des médias: les Harlequin ne permettent pas l'accumulation de capital culturel ni social, mais ils sont au moins bien écrits - nous disait cette femme au foyer de la banlieue parisienne - (E 30), on peut y apprendre à se familiariser avec la langue. La faible lecture, de ce point de vue, et quel que soit son scénario, n'a rien à voir avec la sphère de l'illettrisme; dominée culturellement, elle ne représente pas un univers socialement exclu.

\section{NOTES}

1. Bien qu'elle apparaisse dans ses définitions fonctionnelles comme pure activité ludique.

2. Lesquelles néanmoins, peuvent stimuler la lecture dans le choix de certains thèmes tels que le sport, la vie familiale ou les univers romanesques dominés par les sentiments amoureux ou familiaux.

3. Ouvrages pratiques, sur les voyages, etc. 
Annexes 


\section{Les enquêtes sur la faible lecture}

\section{La méthode}

1 L'orientation qualitative de l'étude a déterminé le choix de deux types de techniques que l'on range traditionnellement dans la catégorie générique des méthodes qualitatives d'observation :

- l'entretien semi-directif, thématique et individuel,

- l'observation directe, fût-elle minimale comme dans notre cas, qui est réalisée auprès des enquêtés mis en situation. Il s'est agit ici d'une interrogation à domicile sur l'aménagement domestique, sur les stocks privés de livres, commentés et historicisés; mais on n'a pas procédé à une observation de type « clinique » des lecteurs en train de lire.

Le déroulement de l'enquête a également suggéré la réalisation de quelques entretiens avec des élèves d'un lycée d'enseignement professionnel (LEP) après les heures de cours, dans les locaux de l'établissement situé en région parisienne. Ces enquêtes présentent l'intérêt de leur contexte: les lycéens y sont interviewés dans leur environnement culturel quotidien - lycée, groupe de copains - et sur un terrain où se conjuguent leur formation professionnelle et la socialisation de leur classe d'âge ; ces enquêtes constituent de ce fait un mode d'observation en situation dans la mesure où la faible lecture de ces enquêtés et leur rapport au livre s'articulent partiellement dans leur expérience sur ce terrain.

3 Les autres entretiens ont été pour la plupart recueillis à domicile ${ }^{1}$, par questionnement individuel ou à deux, chacun des deux époux alternativement. Cette seconde technique suggère des différenciations sexuelles mais appelle également l'évocation spontanée des scénarios familiaux. Une partie substantielle des entretiens, - d'une durée totale d'un peu plus d'une heure et parfois plus - a été consacrée au recueil de données d'observation "ethnographique "; les enquêtés entraînaient spontanément l'enquêteur devant leur bibliothèque, s'ils en possédaient une, présentaient les ouvrages, commentaient la disposition des rayonnages ou l'histoire spécifique de la lecture de chaque livre.

4 Pour chaque entretien, une fiche technique a été dressée qui indique les conditions d'entretien, le repérage sociologique et biographique du lecteur, la description de son 
environnement domestique et la place que le livre y occupe. L'ensemble de ces informations doit être considéré comme la contextualisation du rapport au livre, aussi bien matériel qu'intellectuel (voir exemple de fiche reproduite ci-dessous).

\section{Entretien $\mathrm{n}^{\circ} 5$ : Faible lectrice, 19.5 .85}

\section{Profil de l'interviewée / renseignements personnels.}

CSP (catégorie socioprofessionnelle) : secrétaire Âge : 34 ans ; sexe : féminin

Lieu de résidence : commune rurale à $15 \mathrm{~km}$ d'une grande ville

Niveau d'instruction : BEPC

CSP des parents : gendarme / couturière

Origine géographique : Algérie

10 Situation de famille : vit en concubinage, deux enfants âgés de $21 / 2$ ans et $71 / 2$ ans.

\section{Conditions de l'entretien}

11 L'entretien a eu lieu en fin d'après-midi, après une journée passée ensemble au domicile de l'interviewée, et comme une activité parmi d'autres dans une ambiance très amicale. Nous nous étions assises à côté de la cheminée allumée, le magnétophone posé sur une petite table proche. L'enregistrement est perturbé par les bruits du feu, les horloges qui sonnent, et les enfants qui entrent dans la pièce de temps en temps. Mais ce qui a été perdu en perfection sonore a été compensé par l'ambiance très décontractée qui donnait lieu finalement, à des réponses très naturelles. Dans la mesure où la famille avait récemment emménagé, et que la plupart des livres étaient toujours emballés dans les cartons, la description de la bibliothèque a été impossible. Enfin, l'interviewée mentionne ici et là les noms de ses parents et de son frère, qui la visitaient ce jour-là.

\section{Plan d'observation ethnographique}

12 L'interviewée travaille dans une administration ; elle est syndiquée et lit régulièrement le journal de son syndicat.

13 La vie familiale est très intensive dans le foyer qui reçoit aussi de nombreux amis. L'interviewée et son compagnon passe beaucoup de temps à aménager le foyer: jardinage, menuiserie, bricolage, travaux électriques, plomberie, tapisserie, décoration. Ils cultivent ainsi une sorte de conscience du foyer en tant que lieu de vie, lieu à habiter et que l'on travaille à perfectionner dans ses divers aspects. L'environnement matériel est très riche de leurs propres travaux : une certaine partie de la menuiserie dans la cuisine (assez bien équipée en appareils ménagers, parce que nos interlocuteurs aiment cuisiner) ; certains meubles ont été refaits ou réparés par eux. Tout est aménagé pour le confort et la fonctionnalité.

Nous sommes dans une maison individuelle, avec un jardin et une terrasse de face, ainsi qu'un jardin à l'arrière, les deux jardins étant reliés par un couloir qui traverse la maison. Le rez-de-chaussée comporte une salle de séjour, avec une cheminée, une télévision, un bureau, des canapés et des fauteuils, et la cuisine de l'autre côté du 
couloir. Au premier étage se trouvent deux chambres, un bureau et une salle de bains. Le grenier sera transformé en deux chambres dans deux ou trois années.

15 L'interviewée parle abondamment de ses nombreuses activités créatrices, de ses goûts en activités "de découverte" (cinéma, voyages, musique), ainsi que de sa façon d'organiser son temps extra-professionnel consacré à des tâches ménagères et à des projets plutôt ludiques. Le lien établi entre ses activités de travail manuel (couture etc.) et sa pratique de lecture est manifeste.

\section{L'échantillon (voir ci-après, la liste des personnes interviewées)}

Le corpus des entretiens réalisés recouvre en partie les spécificités sociologiques majeures du faible lectorat mais répond aussi aux nécessités de diversification des scénarios et des catégories socio professionnelles. On trouvera ci-dessous la répartition de ce corpus en fonction des variables couramment utilisées.

\section{Personnes interviewées}

\section{Age Nombre}

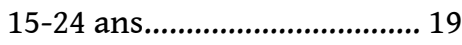


non diplômés

. .8

43 CEP, CAP, BEP, BEPC 34

44 baccalauréat .................................................................... 6

45 études supérieures ........................................................... 7

\section{NOTES}

1. Un ou deux entretiens ont été effectués dans des locaux commerciaux gérés par les enquêtés, pendant les heures de fermeture. 


\section{Liste des personnes interviewées ${ }^{*}$}

\begin{tabular}{|c|c|c|c|c|c|c|c|c|}
\hline Catégorie socio-professionnelle & $\begin{array}{l}\text { GL } \\
\text { ML }\end{array}$ & NL & FL & sexe & âge & résidence & $\begin{array}{c}\text { niveau } \\
\text { d'instruction }\end{array}$ & $\begin{array}{c}\text { situation } \\
\text { de famille }\end{array}$ \\
\hline 1. ouvrière spécialisée & & & $\mathrm{x}$ & $\mathrm{F}$ & 19 & commune rurale & CAP & célibataire \\
\hline 2. enseignant techn. & $\mathrm{x}$ & & & M & 52 & commune rurale & bac +2 ans sup & marié, 2 enfants \\
\hline 3. sans prof. (ex. employée) & $\mathrm{x}$ & & & $\mathrm{F}$ & 45 & commune rurale & niveau bac & mariée, 2 enfants \\
\hline 4. secrétaire & & & $\mathrm{x}$ & $\mathrm{F}$ & 46 & $+100000 \mathrm{~h}$. & licence sc. nat. & mariée, 3 enfants \\
\hline 5. secrétaire & & & $\mathrm{x}$ & $\mathrm{F}$ & 34 & $-20000 \mathrm{~h}$. & BEPC & concubine, 2 enfants \\
\hline 6. enseignante & & & $\mathrm{x}$ & $\mathrm{F}$ & 35 & $+100000 \mathrm{~h}$. & CAPES & célibataire \\
\hline 7. secrétaire & & & $\mathrm{x}$ & $\mathrm{F}$ & 35 & $+100000 \mathrm{~h}$. & BEPC & célibataire \\
\hline 8. cadre administratif & & & $\mathrm{x}$ & $\mathrm{F}$ & 27 & $-20000 \mathrm{~h}$. & bac technique & célibataire \\
\hline 9. représentant & & & $\mathrm{x}$ & M & 36 & banlieue parisienne & CAP & marié, 1 enfant \\
\hline 10. artisan & & $\mathrm{x}$ & & M & 50 & banlieue parisienne & BEP & marié, 2 enfants \\
\hline 11. ouvrier spéc. & & $\mathrm{x}$ & & M & 32 & banlieue parisienne & BEP & marié, 2 enfants \\
\hline 12. employée & $\mathrm{x}$ & & & $\mathrm{F}$ & 32 & banlieue parisienne & BEPC & mariée, 2 enfants \\
\hline 13. femme au foyer & $\mathrm{x}$ & & & $\mathrm{F}$ & 48 & banlieue parisienne & non diplômée & mariée, 2 enfants \\
\hline 14. étudiante & & & $\mathrm{x}$ & $\mathrm{F}$ & 25 & $+100000 \mathrm{~h}$ & DEUG & mariée, 1 enfant \\
\hline 15. orthophoniste & & & $\mathrm{x}$ & $\mathrm{F}$ & 33 & $+100000 \mathrm{~h}$. & études sup. & célibataire \\
\hline 16. technicien & & & $\mathrm{x}$ & M & 33 & $+100000 \mathrm{~h}$. & licence électro. & marié, 1 enfant \\
\hline 17. coiffeuse & & & $\mathrm{x}$ & $\mathrm{F}$ & 58 & $+100000 \mathrm{~h}$. & CEP & mariée, 3 enfants \\
\hline 18. coiffeur & & $\mathrm{x}$ & & $\mathrm{M}$ & 59 & $+100000 \mathrm{~h}$. & CEP & marié, 3 enfants \\
\hline 19. 2 lycéens LEP & & & $\mathrm{x}$ & $\mathrm{M}$ & 17 & banlieue parisienne & CAP & célibataires \\
\hline 20. 2 lycéens LEP & & & $\mathrm{x}$ & $\mathrm{M}$ & 17 & banlieue parisienne & CAP & célibataires \\
\hline 21. 2 lycéens LEP & $\mathrm{x}$ & & $\mathrm{x}$ & $\mathrm{M}$ & 17 & banlieue parisienne & CAP & célibataires \\
\hline 22. 2 lycéens LEP & & & $\mathrm{x}$ & M & 17 & banlieue parisienne & CAP & célibataires \\
\hline 23. 2 lycéens LEP & & & $\mathrm{x}$ & $\mathrm{M}$ & 17 & banlieue parisienne & CAP & célibataires \\
\hline 24. 2 lycéens LEP & $\mathrm{x}$ & $\mathrm{x}$ & & M & 15 & banlieue parisienne & CAP & célibataires \\
\hline
\end{tabular}

\begin{tabular}{|c|c|c|c|c|c|c|c|c|}
\hline Catégorie socio-professionnelle & $\begin{array}{l}\text { GL } \\
\text { ML }\end{array}$ & NL & $\mathrm{FL}$ & sexe & âge & résidence & $\begin{array}{c}\text { niveau } \\
\text { d'instruction }\end{array}$ & $\begin{array}{l}\text { situation } \\
\text { de famille }\end{array}$ \\
\hline 25. employée & $\mathrm{x}$ & & & $\mathrm{F}$ & 20 & commune rurale & CAP & célibataire \\
\hline 26. 2 lyceeens & & & $\mathrm{x}$ & M & 16 & banlieue parisienne & CAP & célibataires \\
\hline 27. horticultrice & & & $\mathrm{x}$ & $\mathrm{F}$ & 27 & $-20000 \mathrm{~h}$ & baccalauréat & concubine \\
\hline 28. ouvrier spéc. & $\mathrm{x}$ & & & M & 21 & $-20000 \mathrm{~h}$. & BEP & célibataire \\
\hline 29. enseignant & & & $\mathrm{x}$ & M & 38 & $-20000 \mathrm{~h}$ & études sup. & marié \\
\hline 30. femme au foyer & & $\mathrm{x}$ & & $\mathrm{F}$ & 64 & banlieue parisienne & non diplômée & mariée, 3 enfants \\
\hline 31. cadre commercial & & & $\mathrm{x}$ & $\mathrm{F}$ & 35 & Paris & BEPC & célibataire \\
\hline 32. commerçant & & & $\mathrm{x}$ & M & 60 & banlieue parisienne & non diplômé & marié, 2 enfants \\
\hline 33. ouvrier & & & $\mathrm{x}$ & M & 22 & $-20000 \mathrm{~h}$ & BEPC & célibataire \\
\hline 34. chômeur & & & $\mathrm{x}$ & M & 51 & $-20000 \mathrm{~h}$. & BEP & célibataire \\
\hline 35. commerçante & & & $\mathrm{x}$ & $\mathrm{F}$ & 60 & banlieue parisienne & non diplômée & mariée, 2 enfants \\
\hline 36. représentant & & & $\mathrm{x}$ & M & 39 & banlieue parisienne & baccalauréat & célibataire \\
\hline 37. retraitée & & & $\mathrm{x}$ & $\mathrm{F}$ & 66 & $+100000 \mathrm{~h}$. & non diplômée & mariée, 1 enfant \\
\hline 38. retraitée & & & $\mathrm{x}$ & $\mathrm{F}$ & 72 & $+100000 \mathrm{~h}$. & CAP & veuve, 6 enfants \\
\hline 39. retraitée & & & $\mathrm{x}$ & $\mathrm{F}$ & 62 & $+100000 \mathrm{~h}$. & CEP & mariée, 2 enfants \\
\hline 40. étudiante & & & $\mathrm{x}$ & $\mathrm{F}$ & 19 & $+100000 \mathrm{~h}$. & baccalauréat & célibataire \\
\hline 41. artisan & $\mathrm{X}$ & & & M & 54 & $+100000 \mathrm{~h}$. & non diplômé & marié, 2 enfants \\
\hline 42. femme au foyer & & & $\mathrm{x}$ & $\mathrm{F}$ & 58 & banlieue parisienne & non diplômée & mariée, 3 enfants \\
\hline 43. ouvrier qualifié & & & $\mathrm{x}$ & M & 57 & banlieue parisienne & niveau bac & marié, 2 enfants \\
\hline 44. sans profession & & & $\mathrm{x}$ & $\mathrm{F}$ & 49 & banlieue parisienne & CEP & mariée, 2 enfants \\
\hline 45. agriculteur & & $\mathrm{x}$ & & M & 54 & commune rurale & non diplômé & marié, 1 enfant \\
\hline 46. agriculteur & & & $\mathrm{x}$ & M & 51 & commune rurale & CEP & marié, 1 enfant \\
\hline 47. agricultrice & & & $\mathrm{x}$ & $\mathrm{F}$ & 48 & commune rurale & CEP & mariée, 3 enfants \\
\hline 48. commerçante & & & $\mathrm{x}$ & $\mathrm{F}$ & 49 & Paris & CAP & mariée, 3 enfants \\
\hline
\end{tabular}




\section{NOTES DE FIN}

*. La numérotation renvoie à celle, initiale, effectuée lors de la collecte progressive des entretiens. Nous l'avons conservée pour faciliter les citations dans le corps de notre exposé.

Abréviations : (GL) grand lecteur: (ML) moyen lecteur; (FL) faible lecteur; (NL) non lecteur. 


\section{Plan d'entretien}

\section{Le livre et la lecture : symbolique et représentations}

- Fonctions et usages sociaux (non directifs); on distinguera le livre de la presse.

- Les lecteurs : qui sont-ils? perception, estimation du rapport lecteur/texte, dispositions à la lecture.

- La lecture dans les pratiques culturelles et dans les media contemporains : complémentarité ou concurrence, diversité des fonctions sociales et culturelles des diverses pratiques, des dispositions de réception et d'interprétation.

- Les genres de lecture connus : hiérarchisation, estimation de la destination des genres, usage social et culturel des genres.

\section{Pratique personnelle de la lecture}

- Livres possédés au foyer :

- Modes d'acquisition : cadeaux, emprunts, échanges (avec qui ?) : pour l'achat: fréquence, lieux, place dans le budget, sélection des genres et des auteurs dans les rayons.

- Genres possédés : inventaire et commentaire, historique de la sélection des genres.

- Destin du livre après la lecture : rangement, revente, échange...

- Historique de la lecture et types de livres et d'imprimés lus (distinguer entre livre et presse)

- Biographie du lecteur : à corréler avec la biographie scolaire, familiale et professionnelle.

- Sélection des lectures : livres, presse, magazines, revues.

- Classement des genres lus : forme éditoriale, contenu, volume, esthétique, auteur.

- Initiative de la lecture : amis, famille, publicité

- Emploi du temps de la lecture : fréquence, intensité, moments, circonstances.

- Lieux de la lecture: foyer (quelle pièce), bibliothèque, transports en commun, coiffeur, médecin.

- Pour la bibliothèque: rythme de la fréquentation, type de bibliothèque, forme de la fréquentation.

- Socialisation de la lecture : circulation des connaissances acquises, réseaux sociaux (travail, famille, voisinage), mémorisation et restitution des connaissances (titres, auteurs, collection, contenu, thème). 
- Place de l'imprimé dans l'environnement matériel: rangement, (lieux et modalités), classement, volume, esthétique.

- Autres pratiques des média: TV, radio (en particulier explorer l'écoute des émissions littéraires et l'usage des informations acquises).

- Autres pratiques culturelles : visite des musées, théâtre, cinéma, vie associative...

\section{Plan d'observation ethnographique}

- Profession et socialisation du travail : collègues, amis, vie syndicale.

-Vie familiale: enfants, visites aux parents, célébrations, proximité ou éloignement des résidences.

- Type de consommation et d'équipement: décrire l'environnement matériel, le mobilier, l'électro-ménager.

- Forme de l'habitat : appartement, maison individuelle... localisation, nombre de pièces, style.

- Loisirs et activités extra-professionnelles.

\section{Renseignements personnels}

1 CSP, âge, niveau d'instruction, CSP des parents et origine géographique, situation familiale.

\section{Conditions d'entretien}

Accueil, lieu d'entretien... 


\section{Extraits d'entretiens}

\section{Entretien 1}

1 Ouvrière spécialisée, 19 ans, titulaire d'un CAP, résidante dans une commune de l'Est de la France, de 500 habitants. Faible lectrice.

2 "En classe, j'avais plus le temps de lire... toutes les semaines on lisait un livre de bibliothèque... comme ça, quand tu n'as rien à faire, tu peux lire... moi, ma lecture, c'est des romans, des romans-photos. Je lis un roman-photo toutes les semaines... mais autrement c'est des magazines. Parfois, je lis un livre un peu plus long, s'il y a une copine qui me dit « il y a un bouquin qui est vachement bien", tiens, il y a trois ou quatre semaines, j'ai lu un bouquin Le pavillon des fous... »

Question : Pour toi, c'est un livre ça?

4 Réponse: "Oui c'est ça, un livre de poche, c'est ce que je lis le plus souvent, un vrai livre je ne le lis pas. Muriel, elle veut toujours me lire des livres, comme elle est en terminale, elle veut que moi aussi je lise des bouquins qu'elle lit, moi je ne les lis pas... je ne comprends pas, je n'ai pas tellement envie de comprendre, envie de lire ça, ça me sert à rien ».

Qu'est-ce que tu appelles un livre qui te sert?

6 "Quelqu'un qui a vécu quelque chose... qui a, comme Christiane F., l'avocat, c'est connu, et il y a un film qui est sorti, comme ça, c'est un truc qui me sert, ça me sert à connaître le monde des drogués, le monde des gens quoi, un autre monde, même si je ne les côtoie pas, ça me fait découvrir autre chose, j'aurais pu le lire deux ou trois fois... Paris au mois d'août, c'est connu je crois, j'avais vu le film alors j'ai acheté le bouquin, mais il est super. Je dépenserais $100 \mathrm{f}$ pour un bouquin comme ça, pour un truc qui m'intéresse, parce que j'aurais aiméle cyclisme, j'aime bien savoir qui a gagné le Tour de France, ça me sert à rien mais ça m'intéresse, j'ai acheté le Livre d'or du cyclisme de 76, 77, 78, j'en ai quatre en tout. Là j'ai acheté le Livre du Racing, il a coûté presque $200 \mathrm{f}$, ça m'a intéressé,... il y a des trucs, les juifs dans la guerre mondiale, ça j'aime bien lire aussi, comme Anne Frank, je l'ai lu même deux fois, des trucs comme ça, ou bien Au nom de tous les miens de Martin Cray...»

7 Comment choisis-tu un livre? 

et bon après je regarde le prix sinon je regarde, je lis l'arrière du livre, bon si ça m'intéresse je le prends, si ça m'intéresse pas, je le laisse. Des trucs intelligents, je ne suis pas... ça n'a pas besoin d'être intelligent, je m'en fous moi... je suis sûre que je comprendrais peut-être un peu, mais je n'ai pas envie de lire un bouquin avec le dictionnaire à côté, parce que sinon je peux lire un dictionnaire aussi, hein, au lieu du bouquin.

Tu vois, chez moi, c'est la vie active maintenant, c'est le travail, métro, boulot, dodo, c'est ça. c'est pas... les loisirs, je n'ai pas envie de les passer en lecture, tu as envie de prendre l'air, de respirer, de courir, j'arrive du boulot, je trouve ça plus intéressant que de rester dans une chambre et de lire, oh ben tu peux $t$ asseoir dehors, mais je ne sais pas, de là un bouquin, le nez dans un bouquin, j'ai un métier qui demande un effort physique, je ne pense pas que tu aies envie de lire... parce que la lecture c'est quand même un effort physique, mais pas physique c'est dans la tête, tu travailles avec la tête, et si tu es crevé physiquement tu n'as pas envie de travailler avec la tête. Si je lis un bouquin, il faut que je puisse le lire, que je sois vraiment dans le bouquin, il faut que je sois seule, que je sois quelque part où je suis relaxe, que je me sente à l'aise. »

11 Est-ce que tu vas parfois dans une librairie pour acheter un livre?

12 "Ça dépend, comment tu dis «librairie »... j'y vais une fois par semaine, même deux fois, en principe quand je vais dedans, $j$ 'achète les magazines, je regarde les livres de poche, mais parfois c'est cher, je n'ai pas tellement envie de dépenser plein de fric pour un bouquin.»

\section{Entretien 17}

15 Artisan coiffeuse, 58 ans, résidant dans une commune de plus de 100000 habitants, Ouest de la France, titulaire d'un CEP. Faible lectrice.

"J'aime lire, assez, des choses qui, à la limite, sont presque un peu inaccessibles, d'ailleurs, Claude me conseille des livres, alors de ce fait là. on lit facilement des choses un petit peu audessus de notre niveau.

17 Je ne suis pas très club, je n'aime pas les contraintes, j'aime bien choisir, bon, on écoute assez les émissions littéraires, comment il s'appelle, l'émission de Pivot.

Le petit Prince, c'est une histoire importante pour moi. Je suis très sensibilisée à cette histoire parce que je trouve qu'elle résume plein de choses et je l'ai aimée très jeune et je l'aime toujours autant en avançant dans le temps. Le petit Prince, si je suis cafardeuse, comme ça peut arriver, je pense à tout le monde, à certaines périodes de vie, l'écoute du Petit Prince m'a toujours beaucoup apporté.

19 Il y a le genre Henri Troyat, Alain Decaux, François Nourrissier ça, j'aimais bien aussi, ça a été offert aux enfants, ça, c'est ce que j'ai offert à mon mari... La racine de l'homme, ça aussi ça c'est ma petite belle fille qui me l'avait offert,... en fait c'est surtout des cadeaux, ou alors des choix que l'on fait, je vous dis, après Apostrophes.

Quand la Chine s'éveillera, j'ai trouvé que c'était un peu statistique... c'est assez ardu à lire, je ne l'ai pas la en entier d'ailleurs, j'ai capitulé et je pense que ce ne sont pas des livres qu'on lit d'une traite, et dans la mesure où l'on a pas beaucoup de temps, ça dépend de l'état d'âme qui est 
le sien, parfois vous avez envie de lire des choses qui détendent et puis d'autres auxquelles on se consacre davantage pour acquérir une connaissance, lion celui-ci ma fille vient de me l'offrir pour la fête des mères: J'ai des choses à te dire. Les chocolats d'Alençon, ce sont des choses vécues ça, François Chalet: je préfere les histoires vécues, les récits, les voyages plutôt que les romans. "

Quels sont les moments où vous vous trouvez la plus disposée à lire ?

"Je ne sais pas, on ne doit pas être un bon exemple, parce que nous, nous ne prenons pas de vacances ou peu. Quand nous prenons des vacances, c'est surtout des vacances randonnées, et bon la saison des vacances n'est pas vraiment lu saison où l'on lit, sauf si on va à la mer ou dans le jardin, mais combien de lundis ou de dimanches peut-on vraiment consacrer.»

Lorsque vous lisez un livre, à quel rythme vous préférez lire?

"Je n'ai pas le temps, j'aimerais lire rapidement mais je n'ai pas le temps, en fait c'est toujours le même leitmotiv qui revient, sinon je pense que j'aimerais lire très vite mais je $n$ ai pus de temps suivi, je n'ai pas d'heures, pas suffisamment d'heures à se suivre."

Vous disiez tout à l'heure que vous n'aimiez pas trop prêter les livres?

"Non, je $n$ aime pas trop prêter les livres... je considère ça comme... d'ailleurs parce que la plupart sont des cadeaux et je ne sais pas, un livre, je trouve que c'est quelque chose de très personnel. Quand je prête un livre à quelqu'un, c'est parce que c'est une amie et que j'ai envie vraiment de lui être agréable, mais je ne le fais pas volontiers. D'ailleurs ce n'est pas pour une question de prix, c'est pour une question d'attache à ce livre, si vous le prêtez, c'est qu'il a retenu votre attention, si vous le faites consulter à une amie, c'est parce que vous pensez qu'il lui serait agréable de le lire... Et puis, il y a un phénomène de cadeaux ou de choix fonctionnels hein, en fait ma bibliothèque est surtout remplie de livres que l'on a choisis personnellement puisque même si c'est un cadeau c'est tout de même un choix... on s'offre pas mutuellement des livres sans se consulter.»

Est-ce que dans votre famille, vos parents, vos frères, vos sœurs lisaient?

"Je ne crois pas, en fait, des quatre que nous étions, je pense que c'est moi qui ai le plus lu, et puis vous savez les enfants qui ont continué leurs études, ont été jusqu'au bac, enfin, ils nous ont ouvert à nous les parents un nouvel horizon, nous n'avions pas fait d'études secondaires; on a lu des livres que lisaient nos enfants. »

\section{Entretien 26}

Deux élèves, d'un lycée d'enseignement professionnel de la région parisienne, âgés de 15 et 16 ans, titualaires de CAP et préparant un BEP.

Quantification variable de la lecture de livres. L'un, Bou Salem, est né en France de parents maliens, le père est chef comptable chez Renault, Francis vit avec sa mère qui est vendeuse (père décédé).

B. S. (Bou Salem) ; F. (Francis) Qu'est-ce que tu fais comme loisirs?

F.: «On a des copains, on joue au football, on est inscrits à un club.» B. S. : "Je fais de la batterie aussi, je vais aller à Pornichet pour faire un stage.»

Et tu lis des revues de musique?

B. S. : «Je ne me rappelle plus... Best..." 
F. : «Le matin, quand je me réveille il y a la radio, et à l'école des fois avec mon walkman, ou alors le soir, il me faut toujours de la musique moi dans la vie.»

Et au niveau des lectures?

F. : «Des bandes dessinées, tous les soirs j'en lis une, Mickey, des trucs comme ça. Je les achète au bureau de tabac. J'ai lu aussi Vingt mille lieues sous les mers, je l'ai lu en entier ça.»

Combien de livres lis-tu par an?

F. : «Je ne peux pas les compter, je ne sais pas 7 à 8.»

Qu'est-ce que tu comptes comme livres là?

F. : "Que des bandes dessinées, et des livres de musique."

Et des livres pornos, vous en regardez aussi?

Les deux : «Ben évidemment » (rires)

F. : «Moi, les livres, je ne les revends pas à mes copains, mais habituellement, quand je vais au marché, il y a un marchand de livres, il achète les vieux livres très peu recherchés et moi je les vends $15 f$, $20 f$. Je ne les garde jamais. »

B. S. : «Moi je les garde souvent et quand un copain il vient me dire: «Tu n'as pas un Strange à me prêter?", et ben je lui prête."

Qu'est-ce qui te paraît le plus important, un journal, une revue ou un livre?

BS. : «Un journal, ça se lit plus, on s'instruit plus qu'un livre et on sait plus de choses... parce qu'il y a des mots qu'on ne connaît pas et qu'on voit dans les journaux. "

C'est pas inventé ? Et dans un livre ce n'est pas le cas?

B. S. : « Pas dans tous, ils racontent des histoires vraies et d'autres non.»

Tes parents te passaient quelquefois des livres?

B. S. : « Non, ils s'en foutent. »

F. : «Si, ils me passent des livres, mais je ne veux pas les lire, ils sont épais comme ça, ça me décourage.»

\section{Entretien 27}

Horticultrice 27 ans, résidante dans une commune du midi de la France de moins de 20000 habitants, titulaire du baccalauréat. Faible lectrice.

Parle-moi des livres que lu as chez toi.

"Disons qu'il y a beaucoup de livres qu'avaient achetés mes parents, et comme j'ai habité chez eux très tard, c'étaient les livres de la famille et c'était mélangé, une bibliothèque très complète, il y avait des essais, des romans, de la poésie, des livres d'art, des livres étrangers. " Est-ce qu'il y a eu des moments dans ta vie où tu as lu plus que maintenant?

57 "Oui, oui, dans ma famille mes parents lisaient beaucoup, ils achetaient des bouquins et il y avait une bibliothèque énorme. Toute petite j'ai commencé à lire énormément, je me rappelle, je me réveillais à $6 \mathrm{~h}$ du matin, avant d'aller à l'école je lisais pendant une heure avant de me lever." 

en fait que ça fait partie de tout un processus mental, d'un malaise intérieur peut-être; j'ai l'impression que c'est en relation avec une rupture d'équilibre. Dans le temps où j'étais au lycée, j'étais bien canalisée, je savais où j'étais et je savais ce qui me plaisait et j'arrivais à lire, et puis après, j'ai eu des bouleversements, ma vie a changé, j'ai pas mal bougé et c'est à partir de ce moment-là où j'ai commencé à beaucoup moins lire... Ça se faisait après sans guère de livres, je lisais des textes... j'ai lu pas mal de textes isolés.

Et pourtant j'ai un immense désir de lire, vraiment ce qui me manque c'est de lire, de lire, de lire, parce que plus ça va, plus j'avance en âge et moins j'ai de références, avant j'avais des tas de références et puis on oublie."

61 Quand tu dis que tu ne lis plus, ça veut dire que tu ne lis plus de livres, mais autrement tu lis quand même des journaux...?

62 "Oui le journal je l'achète presque tous les jours, des revues, j'achète Elle, tout le monde se moque de moi, tous mes amis, ils trouvent ça idiot. »

"Alors c'est ça le problème, c'est peut-être aussi lié à une question de temps... il y a beaucoup de choses dans ma vie qui me distraient de la lecture, alors je n'ai jamais vraiment le temps de lire, je suis toujours en train de courir... "

"Mais non, c'est ça qui est triste, les gens que je fréquente ici, des gens qui ne lisent pas, donc ils ne parlent jamais de bouquins ils ne vont pas au cinéma, ou quand ils vont voir des films, c'est des Kung $\mathrm{Fu}$, des choses comme ça, des succès commerciaux, donc il $n$ y a pas de conversation à axer là-dessus. Ce dont ils parlent, c'est des BD. Le loup garou des îles, ça, peut les faire parler, moi pas du tout. Voilà. c'est ça, c'est que je me trouve plus du tout dans un milieu qui me pousse à lire... Si j'invitais par exemple un intellectuel ou quelqu'un qui écrit ou qui lit beaucoup, peutêtre que ça me stimulerait."

"Mes bouquins à moi, ils sont mélangés aux bouquins du reste de la famille, et puis il y a mes bouquins d'enfance qui sont dans des caisses, que je sortirai un jour pour mes enfants... mais... un truc que j'ai oublié, c'est que de savoir, de voir mes bouquins là... a un côté rassurant, ça me rassure de les voir là posés sur ma table, ils sont prêts à être ouverts, à être lus, mais ça ne me pousse pas du tout à les ouvrir (rire).»

Alors ça représente quelque chose d'important pour toi la lecture?

"Oui, oui c'est un de mes grands regrets, c'est comme de ne pas jouer de piano... j'ai l'impression qu'il faut que je réapprenne à lire, que je réapprenne à me concentrer. Il faut que je réapprenne l'habitude de la lecture que j'ai perdue. "

D'après toi, qui sont les gens qui lisent beaucoup?

"Ils font partie de la classe de ceux qu'on appelle "les intellectuels", tout le corps professoral, littéraire, pas tous, les profs de philo, de français, de lettres, d'histoire, et puis après tu as des passionnés de la lecture. C'est pas un vrai loisir la lecture, c'est un truc qui fait partie justement, qui devrait faire partie de la vie quotidienne des gens. " 


\section{Entretien 30} pas l'acheter... j'aime un livre qui est bien relié, il a une belle reliure, tout ça, c'est très joli, parce que quand on met ça dans une bibliothèque, c'est bien plus beau qu'un livre ordinaire... la belle lecture et le beau livre en même temps, l'un n'empêche pas l'autre. Des fois ça m'arrive, j'ai des bouquins Harlequin, des petits romans à l'eau de rose, bon j'en lis beaucoup, mais je n'ai pas l'impression que ça me fait faire un boum intellectuellement, c'est juste pour m'évader un peu, me détendre... Ils sont bien écrits et c'est pour ça qu'il faut lire les livres bien écrits si on veut vraiment s'instruire un petit peu... C'est des petites histoires vraiment banales, toujours pareilles, mais elles sont bien écrites, mais c'est pas ça qui m'apporte vraiment : quand je lis un livre d'un grand auteur bien sûr, j'ai l'impression que ça m'a apporté plus. »

\section{Entretien 33}

Ouvrier pâtissier temporaire, 22 ans, résidant dans une commune de moins de 20000 habitants du midi de la France, titulaire du BEPC. Faible lecteur.

83 "Mon père a beaucoup de livres, moi je n'ai que des BD, je lis mais pas trop... du temps que j'achetais Spatial... ça va mais ça s'arrêtait là, j'allais dans une librairie, je n'allais pas voir les livres, j'allais de suite voir les $B D$ ou des trucs de musique, mais s'il y a des livres, des romans 
tout ça, je n'allais pas les voir, même pas par curiosité, parce que je ne suis pas curieux de ça. Mon père à une époque, il lisait des romans policiers, des trucs d'espionnage, après il a fait, enfin, il essaye de faire sa petite bibliothèque, des classiques, Victor Hugo tout ça, tous ces gens-là, ces gens intéressants mais qui ne m'intéressent pas ou que je ne veux pas m'intéresser. Non, lui il fait sa petite bibliothèque, il est abonné, "... France Loisirs... Moi, quand arrivent les titres, je dis rien, je regarde plus facilement la télé qu'un bouquin. Je n'ai jamais lu, je n'ai jamais aimé, jamais, jamais, si quand j'étais jeune, j'ai lu peut-être un livre, c'est Robinson Crusoé, c'est tout, ça ne m'a jamais, jamais, ça m'a toujours arrêté... le français ça n'a jamais été quelque chose... Oh oui, si j'ai le temps je resterais là à lire des magazines, ça oui, pour moi, je n'aime pas lire tout ce qui est roman, tout ce qui est auteur qui écrit une histoire, soit réelle, soit imaginaire les magazines oui, tout ce qui est reportage, tout ce qui est sur les recherches scientifiques et sur l'histoire, sur les animaux, surtout ce qui a rapport avec la terre, ça d'accord... d'horrible, en quelques images. Mais quand j'ai vu le truc j'avais vraiment déliré dessus. C'était un mec qui passait au tribunal en BD : "Alors vous avez tué, donc vous devez être tué». Alors le mec on le passe à l'échafaud, et puis le bourreau il est habillé et tout, il le tue quoi, il lui fait tomber le truc, donc lui il a tué, donc il doit se tuer... Et puis la dernière image, tu vois le mec, l'assassin soi-disant, il tombe, et puis le bourreau ça ne s'arrête jamais, l'un qui a tué, l'autre... j'ai trouvé ça génial.»

\section{Entretien 36}

Représentant de commerce multi-cartes, 39 ans,
titulaire d'un baccalauréat technique. Faible lecteur.

91 choses, la musique, c'est facile, on a juste à écouter, on peut découvrir ce que l'on veut dans la musique, alors qu'un livre, il faut essayer de découvrir effectivement ce que l'auteur a voulu. Lorsque vous êtes jeune, vous lisez, ça vous apprend un peu le français, ça vous apprend à écrire 
des choses. Moi j'ai lu trop tard, c'est pour ça que je suis pas très fort en français et que par la suite, lorsqu'on a mon âge, on fait beaucoup moins attention, on a beaucop moins de mémoire, et la mémoire ça se cultive... Le livre, ça a une image de la culture par excellence.

92 Moi un livre qui me passionne, je le lis dans la journée, enfin je le lisais dans la journée, maintenant... je vais partir en vacances, je vais lire le livre d'Yves Montand, des choses comme ça, des choses assez faciles. Enfin, je n'en achète plus, c'est mes parents qui maintenant sont en retraite en achètent beaucoup, ils sont abonnés à des tas de clubs et ils me les prêtent... Je passe chez mon père, je vais lire le Canard enchâ̂né... si j'y vais manger à midi, mais je n'ai vraiment plus le temps, c'est pas la peine que je l'achète. C'est vrai que je n'achète plus de quotidien. Si,je vais acheter L'usine nouvelle, des choses comme ça, pour mon travail, mais c'est professionnel. »

Quel est pour vous le livre par excellence?

94 "Je suis beaucoup plus attiré par l'écrivain du XIX siècle... celui qui cultive, c'est ça... de toute façon, en plus c'est une éducation parce que qu'est-ce qu on vous apprend à l'école, enfin surtout à mon époque, qu'est-ce qu'on nous faisait lire? des livres du XIX siècle,"

Et la bande dessinée? "Je n'en lis plus du tout, oui, quand j'étais jeune, je lisais Mickey, Spirou, parce que mon père m'en ramenait et puis je crois que c'est indispensable, un enfant qui ne lit pas de bandes dessinées c'est pas logique.»

97 Les encyclopédies, les dictionnaires?

98 "La première chose que j'ai fait lorsque je me suis installé chez moi, c'est d'acheter 3 dictionnaires et un dictionnaire plus approfondi sur le vocabulaire, c'est vraiment indispensable dans un foyer."

Comment évaluez-vous le lecteur idéal?

"Quelqu'un qui lit tous les jours... C'est-à-dire, des bandes dessinées, non, ça n'est pas un lecteur ça... Et puis quelqu'un qui est capable de vous parler de ce qu'il a lu et de le finir.»

\section{Entretien 38}

101 Secrétaire dactylo retraitée, 72 ans, résidant dans une agglomération de plus de 100000 habitants, titulaire d'un CAP. Faible lectrice.

102 "Je lis dans la journée, et le soir, je suis là, je lis un peu, je lis surtout avant de me coucher. Des fois, je tombe sur des livres. Là j'en ai que mon fils m'a prêté, un livre de droit. Ça m'intéresse parce qu'il y a des fois des choses qu'on ne connaît pas... Voilà on parlait du divorce parce que mon fils veut savoir où il en est de son divorce, alors il l'a acheté pour connaitre un peu tous les droits. Ils expliquent tout dessus.

103 J'aime lire tes livres sur la guerre, parce que je l'ai beaucoup vécue cette guerre-là de 40-45, j'aimais relire tout ça et revoir les épisodes qui se sont passés... Les romans, comme ça, d'amour, je n'aime pas, je ne lis pas tellement les livres policiers, parce que je trouve que c'est trop long, et comme c'est écrit très fin, ça m'abîme les yeux; il faut que je lise des livres à gros caractères.

Il y a beaucoup de choses qui m'attirent. Quand je finis de regarder un film qui parle, mettons, de gens que je connais, des artistes que je connais, j'aime savoir ce qu'ils font, et ce qu'ils ont dit d'après la presse que j'ai entendue, alors j'écoute et puis après je lis... J'ai acheté un livre qui m'a beaucoup intéressée, c'est le livre d'Elie Wiesel, je l'ai lu et je l'ai passé à ma fille; elle a L'oiseau 
bariolé, elle finit de le lire et elle me le passe aussi. Oui, on se passe des livres, des fois c'est moi qui les achète, des fois c'est elle.

105 J'ai beaucoup lu aussi sur le cancer, comme ma fille a eu le cancer... J'ai des livres sur la médecine, parce que je suis allergique aux médicaments, aux antibiotiques, alors quand on me donne un médicament je regarde et... Depuis la mort de mon mari, seulement, je me suis mise à lire beaucoup, avant je n'avais pas le temps, quand je travaillais, j'ai élevé mes enfants... Pour moi la lecture représente un moment de détente, et... puis ça nous apprend à connaître un peu ce qui se passe dans la vie courante... Je sais pas, pour moi ça m'instruit et ça me détend en même temps. »

106 Est-ce qu'il vous arrive d'être stimulée à acheter un livre par la radio ou par un autre moyen?

"Oui, des fois, à la radio et quand j'assiste à l'émission de... Apostrophes, quand il parle des livres, je prends note des livres qui m intéressent. J'ai une liste de livres que je ne suis pas arrivée à acheter parce que je ne les ai pas trouvés. Des fois, quand je passe devant une librairie, je demande, je tire mon petit papier et je demande s'ils ont tel livre. Ils le cherchent mais des fois ils ne le trouvent pas... Mais je ne vais pas spécialement, c'est quand je suis devant une librairie que je pense que j'ai la liste des livres que j'ai relevés et je demande.»

108 Quels genres de livres préférez-vous lire ? Comment les classez-vous?

109 "Un intérêt général, ça m’intéresse, sur la personne elle-même, pour ce qu'elle a vécu et ce qu'elle a fait ça non, je n'ai pas lu. Mais, je ne suis pas tellement littéraire, parce qu'il doit y avoir des bons livres que... un peu meilleurs que ceux que j'ai lus, alors je ne peux pas dire comment je les classe, je ne sais pas comment dire."

110 Est-ce que vous parlez des livres que vous avez lus avec certaines personnes?

111 "Oui... au fond la lecture ça aide la personne, moi depuis que je lis, je suis beaucoup aidée par la lecture, je suis mieux informée je suis plus au courant des lois, de ce qui se passe,... il y en a qui dramatisent tout, mais moi quand je lis et que je vois qu'il y a des gens qui s'en sortent de telle ou telle façon... ça m'encourage.»

\section{Entretien 41}

112 Artisan-tailleur, 54 ans, résidant dans une agglomération de plus de 100000 habitants, non diplômé. Moyen lecteur.

113 «La lecture... je lis beaucoup, énormément, moi, tout ce qui se présente... la télé, je n'aime pas tellement, je regarde les informations et puis si le début du film me plait je reste, sinon je vais faire un puzzle ou bien je vais lire. Je lis tous les soirs jusqu'à ce que mes yeux se ferment. »

114 Que représente la lecture pour vous?

115 "J'aime bien ce qui est vécu, les histoires vécues, pas les romans sur l'Himalaya ou sur la Créée, des trucs comme ça, j'aime bien les histoires vraies... Je ne suis pas amateur des académiciens. Je n'ouvrirai jamais un livre de Françoise Sagan ou un truc comme ça... J'ouvre le livre si il me capte, si il me séduit au bout de la cinquantième page.

116 A part les livres que j'achète, je suis abonné à une bibliothèque, quand je les finis, je les offre à la bibliothèque. Des fois quand je n'ai pas l'occasion.

117 j'entends parler d'un livre, je vais là-bas et je le demande, ils l'ont.... des fois ils l'achètent exprès pour moi, parce que je leur fais cadeau des livres." 
" J'ai jamais été disposé à lire, non c'est drôle, j'ai jamais été passionné par la lecture, d'un roman tout au moins. Un reportage, une chronique politique des choses comme ça oui, j'aime. Je lis pas beaucoup, hein, je lis une demi-heure par jour, mais pas plus, je lis plutôt le journal, les commentaires sportifs, les commentaires politiques, les chroniques agricoles et ça s'arrête là. Peut-être que si je lisais un roman, je pourrais être pris, mais pour l'instant je ne suis pas passionné. Quand j'ai commencé un livre, je suis très longtemps à le lire. J'ai presque pas lu, quelques livres de Malraux peut-être quelque chose comme ça... Le nœud de vipères, enfin c'est 
si peu que ça vaut même pas la peine d'en parler,... quand on lit un livre tous les cinq ans... Les gens qui lisent beaucoup sont des gens qui ont poursuivi des études plus longtemps, qui ont été davantage en contact avec les études, avec les livres en général... Le fait que l'agriculteur par luimême a une activité physique assez importante... je ne vois pas un agriculteur s'arrêter au milieu de la journée pour aller lire quelque chose, ça ne serait que le soir, et le soir la fatigue l'emporte». 


\section{Bibliographie}

BARBIER-BOUVET Jean-François, POULAIN Martine. - Publics à l'œuvre. - Paris : La Documentation française, 1986. $-296 \mathrm{p}$.

BELLEVILLE Pierre. - Les attitudes culturelles des travailleurs manuels. - Centre de culture ouvrièreest, DGRST, 1977.

BELLEVILLE René. - Pour la culture dans l'entreprise : rapport de mission au ministère de la Culture. Paris : La Documentation française, 1982. - 78 p.

BENICHOU Jean-Pierre, ESPÉRANDIEU Véronique, LION Antoine. - Des illettrés en France, rapport au Premier ministre. - Paris : La Documentation française, 1984. - $157 \mathrm{p}$.

Les bibliothèques en France: rapport au Premier ministre établi en juillet 1981 par un groupe interministériel présidé par Pierre Vandevoorde. - Paris : Dalloz, 1982. - 447 p.

BOURDIEU Pierre. - La distinction : critique sociale du jugement. - Paris : Ed. de Minuit, 1979. - 672 p.

BOURDIEU Pierre. - Les trois états du capital culturel, Actes de la recherche en sciences sociales, 1979. $-\mathrm{n}^{\circ} 30$, pp. 3-6.

CERTEAU Michel de. - Lire, un braconnage, l'Invention du quotidien,. - Paris : Bourgois 10/18, t. 1, pp. 279-296.

Colloque lecture et bibliothèques publiques : Actes du colloque tenu à Hénin-Beaumont en novembre 1982. - Lille : Office régional de la culture, 1983. - $361 \mathrm{p}$.

Enquête sur les Français et la lecture, Le Monde [des livres], octobre-novembre 1984.

L'expérience et l'image des bibliothèques municipales. Enquête menée en 1979 par l'ARCmc, Bulletin des bibliothèques de France, 1980, t.25, $\mathrm{n}^{\circ} 6$.

FONTAINE M. - Le livre et la lecture en France aujourd'hui, Forum-France. mars 1984, pp. 2-7.

FOUCAMBERT Jean. - La Manière d'être lecteur. - MDI, AFL, 1976.

FOUCAMBERT Jean. - Lire c'est vraiment simple quand c'est l'affaire de tous. - OCDL, 1982.

FURET François, OzoUf Jacques. - Lire et écrire : l'alphabétisation des Français de Calvin à Jules Ferry. Paris : Ed. de Minuit, 1977, 2 vol., pp. 394-379.

HOGGART Richard. - La Culture du pauvre : étude sur le style de vie des classes populaires en Angleterre. - Paris : Ed. de Minuit, 1970. - 420 p.

LAE Jean-François, NOISETTE Patrick. - Je. tu, il apprend. - MIRE, ministère des Affaires Sociales, 1986. 
LAFARGE Claude. - La valeur littéraire. - Paris : Fayard, 1983.

LEENHARDT Jacques, JoszA Pierre. - Lire la lecture, essai de sociologie de la lecture. - Paris : Sycomore, 1982.

MAUSS Marcel. - Essais de sociologie. - Paris : Seuil, 1969. - 256 p.

NAFFRECHOUX Martine, PARMENTIER Patrick, PORTO-VASQUEZ Fernando. - Trois études sur la lecture: enquête et analyse réalisées par le Gides, 1981. - 451 p.

L'Eil à la page : enquête sur les images et les bibliothèques réalisée pour la Direction du livre par le Gides (Groupe inter universitaire de documentation et d'enquêtes sociologiques). - Paris : BPI, 1985. - $346 \mathrm{p}$.

PANSU Alain. - La lecture dans les comités d'entreprise, Cahiers de l'animation, 1982, III, n 37, pp. 57-63.

PINGAUD Bernard, BARREAU Jean-Claude. - Pour une politique nouvelle du livre et de la lecture, rapports au ministre de la Culture. - Paris : Dalloz, 1982. - 300 p.

Pratiques de la lecture, sous la direction de Roger Chartier. - Paris : Ed. Rivages, 1985. - 241 p.

Pratiques culturelles des Français. - Secrétariat d'État à la Culture, 1974, 2 vol.

Pratiques culturelles des Français, description socio-démographique, évolution 1973-1981, ministère de la Culture, service des études et recherches. - Paris : Dalloz, 1982.

Rapports complémentaires :

La lecture chez les ruraux, 1977.

La lecture dans les sondages, 1978.

Le livre, extrait de Pratiques culturelles des Français, 1982.

L'intensité de la lecture, analyse de segmentation, 1984.

Brefs repères sur les faibles lecteurs, 1984.

La lecture, analyse factorielle, 1984.

Données complémentaires sur les non-lecteurs, les faibles lecteurs et les grands lecteurs, 1984.

ROBINE Nicole. - Pour une unification des champs de recherche sur la lecture et le livre, Bulletin des bibliothèques de France, 1980, t. 25, n 16, pp. 205-208.

ROBINE Nicole. - La lecture des livres en France à travers les enquêtes nationales et locales, Cahiers de l'animation, 1983, II, 40, pp. 58-73.

ROBINE Nicole. - Les jeunes travailleurs et la lecture. - Paris : La Documentation Française, 1984.

ROUET François. - Le livre, Notes et études documentaires, 1979, № 4535-4536, pp. 13-25.

THIESSE Anne-Marie. - Le roman du quotidien, lecteurs et lectures populaires à la Belle Époque. - Paris : Le Chemin vert, 1984. - $272 \mathrm{p}$.

SEIBEL Bernadette. - Bibliothèques municipales et animation. - Paris : Dalloz, 1983. 